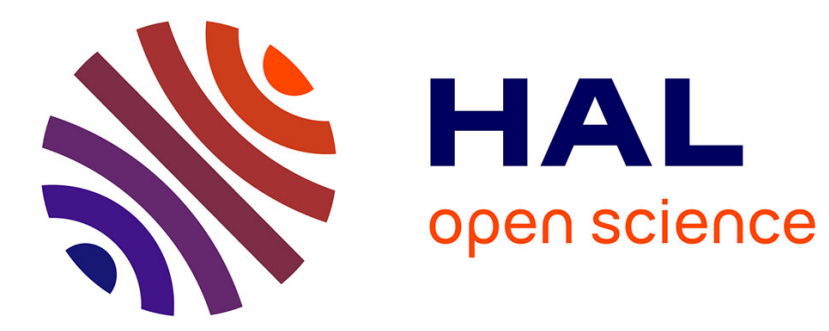

\title{
Mechanical Control of Morphogenesis by Fat/Dachsous/Four-Jointed Planar Cell Polarity Pathway
}

Floris Bosveld, Isabelle Bonnet, Boris Guirao, Sham Tlili, Zhimin Wang, Ambre Petitalot, Raphaël Marchand, Pierre-Luc Bardet, Philippe Marcq, François Graner, et al.

\section{To cite this version:}

Floris Bosveld, Isabelle Bonnet, Boris Guirao, Sham Tlili, Zhimin Wang, et al.. Mechanical Control of Morphogenesis by Fat/Dachsous/Four-Jointed Planar Cell Polarity Pathway. Science, 2012, 336 (6082), pp.724-727. 10.1126/science.1221071 . hal-02463702

\section{HAL Id: hal-02463702 https://hal.science/hal-02463702}

Submitted on 1 Feb 2020

HAL is a multi-disciplinary open access archive for the deposit and dissemination of scientific research documents, whether they are published or not. The documents may come from teaching and research institutions in France or abroad, or from public or private research centers.
L'archive ouverte pluridisciplinaire HAL, est destinée au dépôt et à la diffusion de documents scientifiques de niveau recherche, publiés ou non, émanant des établissements d'enseignement et de recherche français ou étrangers, des laboratoires publics ou privés. 
Mechanical control of tissue morphogenesis by the Fat/Dachsous/Four-jointed planar cell polarity pathway

Floris Bosveld ${ }^{1, *}$, Isabelle Bonnet ${ }^{1, *}$, Boris Guirao ${ }^{1, *}$, Sham Tlili ${ }^{1,}$, Zhimin Wang ${ }^{1}$, Ambre Petitalot $^{1}$, Raphaël Marchand ${ }^{1}$, Pierre-Luc Bardet ${ }^{1}$, Philippe Marcq ${ }^{2}$, François Graner ${ }^{1}$, Yohanns Bellaïche ${ }^{1, \#}$

1. Polarity, Division and Morphogenesis Team, Institut Curie, CNRS UMR 3215, INSERM U934, 26 rue d’Ulm, 75248 Paris Cedex 05, France.

2. Laboratoire Physico-Chimie Curie, Institut Curie, CNRS UMR 168, Université Pierre et Marie Curie, 26 rue d’Ulm, 75248 Paris Cedex 05, France.

* These authors contributed equally to this work.

$\$$ Present address: Matière et Systèmes Complexes, Université Paris Diderot, CNRS UMR 7057, 10 rue Alice Domon et Léonie Duquet, 75205 Paris Cedex 13, France

\# Corresponding author: yohanns.bellaiche@curie.fr 


\section{Summary}

During animal development, several planar cell polarity (PCP) pathways control tissue shape by coordinating collective cell behavior. It remains largely unknown whether and how PCP pathways modulate mechanical forces to drive tissue deformation. Here, we characterize the morphogenesis of the Drosophila dorsal thorax epithelium by multiscale imaging and we decipher how the Fat/Dachsous/Four-jointed PCP pathway controls tissue shape. We found that the proto-cadherin Dachsous is planar polarized within a domain of its tissue-wide expression gradient. Furthermore, we showed that Dachsous polarizes the myosin Dachs, which in turn promotes an anisotropy in cell junction tension. By combining physical modeling with quantitative image analyses, we determined that such anisotropic tensions define the pattern of local tissue contraction rates that shape the epithelium mainly via oriented cell rearrangements. Our results establish how tissue planar polarization coordinates the local changes of cell mechanical properties to control tissue morphogenesis.

\section{One sentence summary}

Planar polarization of the proto-cadherin Dachsous and the myosin Dachs locally regulates the anisotropy of cell junction tension to control tissue morphogenesis. 
Planar cell polarity (PCP) pathways have emerged as fundamental regulators of tissue morphogenesis in both vertebrates and invertebrates (1). In parallel, numerous studies have revealed that the local regulation of cell mechanical properties plays a fundamental role in tissue morphogenesis (2). A central challenge is now to understand whether and how PCP pathways control local cell mechanical properties to drive global tissue deformation, hence morphogenesis.

The Drosophila Fat/Dachsous(Ds)/Four-jointed(Fj) PCP pathway plays fundamental roles in the regulation of tissue growth, the orientation of cell polarity across tissue and the regulation of morphogenesis (1,3). fat and $d s$ encode proto-cadherins, while $f j$ encodes a Golgi kinase modulating Fat/Ds binding (4-9). In many Drosophila epithelial tissues, $d s$ and $f j$ are expressed in tissue-wide opposing gradients $(5,6,10,11)$. Fat and Ds are reported to be homogeneous at the cell membrane $(11,12)$. Yet, the heterophilic binding of Fat and Ds between adjacent cells is proposed to generate a tissue-wide polarity $(8,9,11,13-16)$. Through a poorly understood mechanism implicating Fat signaling, this polarity is translated into the asymmetric distribution of the myosin Dachs, which controls cell division orientation $(13,17-$ 20). The role of the Fat/Ds/Fj pathway in tissue morphogenesis remains to be characterized. It has been mostly studied using indirect measurements such as the shape of clones and cell division orientation $(20,21)$; conversely, measurements of tissue flow have so far revealed its role in tissue rotation (22) but not in tissue shape changes. Moreover no study has directly assessed whether, where and how the Fat/Ds/Fj pathway impacts on local cell mechanical properties to drive morphogenetic movements.

We implemented a multiscale imaging method to record the morphogenesis of the Drosophila dorsal thorax during metamorphosis (23). This monolayered epithelium is composed of a posterior region, the scutellum, and of a large anterior region, the scutum (fig. S1A-B). Cells were labeled with E-Cadherin:GFP and the tissue was imaged from 11 hours after pupa 
formation (hAPF) onwards, by acquiring high-resolution 3D stacks tiling the thorax at each time-point (Fig. 1A, Movie S1). This multiscale imaging enabled to follow the $\sim 10^{4}$ cells of the tissue over several cell cycles with an unprecedented dynamics: 5 min temporal resolution over $26 \mathrm{~h}$ of development and $0.32 \mu \mathrm{m}$ spatial resolution over the $750 \times 700 \mu \mathrm{m}^{2}$ of the tissue. At the cell-scale, the spatial resolution $(\sim 300$ pixels/cell $)$ and the 5 min temporal resolution facilitated the determination and the tracking of cell apex areas, cell shapes, divisions, cell rearrangements and apoptoses (Fig. 1B-D, fig. S1C-D, Movie S2). At the tissue-scale, we measured, by image correlation, local tissue flow over the whole tissue at once using a length scale of 10-20 cells and a time scale of $2 \mathrm{~h}$, both providing a good signal-to-noise ratio. This revealed the different periods of tissue development (fig. S2, Movie S3). In particular between 17:20 and 21:20 hAPF, the velocity map showed morphogenetic movements both in the scutum and the scutellum (shaded regions, Fig. 1E). Such tissue flow promoted tissue contraction and elongation in the lateral scutum resulting in its anterior-posterior elongation and in the medial scutellum resulting in its medial-lateral elongation (Fig. 1F, scutellum in yellow). Collectively our multiscale imaging and measurements illustrate the elaborate cellular properties and coordinated tissue flows that shape the dorsal thorax. They provide an important resource to investigate how signaling pathways control tissue morphogenesis.

Looking for possible regulators of these morphogenetic movements, we observed that $d s$ and $f j$ were both expressed in the scutellum (fig. S3), their opposing gradients forming a $\Lambda$-shaped domain in each hemi-scutellum (Fig. 2A-A'). To understand the role of Fat/Ds/Fj signaling in tissue morphogenesis, we thus focused our analysis on the contraction and elongation taking place in the scutellum. The average tissue deformation rate map between 17:20 and 21:20 hAPF revealed that tissue deformation in the scutellum was not homogenous along the $d s$ and $f j$ gradients (Fig. 2B, S4). This suggests that Fat/Ds/Fj activation might vary along the $d s$ and $f j$ gradients and emphasizes the need to define where the Fat/Ds/Fj pathway is active to 
investigate its role in morphogenesis. Given the genetic evidence indicating that the subcellular localization of the myosin Dachs is controlled by Fat activity $(17,18,24)$, we generated a Dachs:GFP (D:GFP) rescue construct and imaged it in the scutellum (Movie S4). This revealed that: 1) D:GFP was polarized in the $\Lambda$-shaped domain where the opposing expression gradients of $d s$ and $f j$ meet (Fig. 2C); 2) within the regions of D:GFP planar polarization, D:GFP enriched cell boundaries were aligned with each other leading to D:GFP planar polarity lines (arrowheads in Fig. 2C).

To investigate how Dachs polarity is regulated in the $\Lambda$-shaped domain, we analyzed the role and the localization of Fat, Ds and Fj. Loss of $d s$ or $f t$ function as well as overexpression of $f j$ disrupted D:GFP polarization (Fig. 2D-E, fig. S5A-D). Furthermore, in agreement with the fact that D:GFP was mostly polarized in the region where the $d s$ and $f j$ gradients were opposed, clonal overexpression of either $d s$ or $f j$ induced repolarization of D:GFP only in regions where $f j$ or $d s$ were expressed, respectively (fig. S5E-F). We then analyzed the subcellular localization of Fat, $\mathrm{Ds}$ and $\mathrm{Fj}$ in relation with the Dachs polarization domain (fig. S5G-I'). Doing so, we established that in regions where D:GFP was polarized, Ds was also polarized (Fig. 2F, fig. S5G-G'). Importantly, Ds polarization required the $f j$ expression gradient and Fat activity, but it was independent of Dachs activity (fig. S5J-M'). Both Ds and D:GFP polarized towards high levels of $f j$ expression and colocalized at the junctions (Fig. 3A-C'). Furthermore D:GFP can pull-down the Flag:Ds intracellular domain (Fig. 3D). Altogether our results revealed that Ds is planar polarized in the region where the opposing gradients of $d s$ and $f j$ meet. In turn, the interaction between Ds and Dachs promotes the polarization of Dachs (fig. S6 for model).

The ectopic localization of Dachs is shown to reduce cell apex area, suggesting its possible implication in cell apex constriction (20). In order to directly assess whether the Fat/Ds/Fj pathway modulates cell mechanical properties via Dachs polarization, we performed laser 
ablation of cell junctions and observed that the tension of junctions enriched in D:GFP was on average 2-fold higher compared to junctions devoid of D:GFP (Fig. 3E-F', Movie S5). To further confirm that Dachs polarization leads to anisotropic junction tension, we generated fat clones that induced an increased polarization and an accumulation of Dachs in mutant junctions facing the wild-type ones (fig S7A,C). Such junctions presented an increased tension that depends on Dachs activity (fig S7B). Finally MyosinII was neither significantly polarized along the Dachs polarity lines nor at the fat clone boundaries (fig. S7D-H). This shows that Dachs polarity regulates junction tension anisotropy, and suggests that the opposing $d s$ and $f j$ gradients generate an anisotropic tension along the lines of Ds and Dachs polarity.

To investigate whether the anisotropic tensions along the Dachs polarity lines control tissue morphogenesis, we developed a physical model that provides a general method to analyze the morphogenetic contribution of a specific signaling pathway within an elaborated morphogenetic movement (SOM). It predicted that Dachs anisotropic tension is sufficient to modulate the local contraction rate. Furthermore, it provided a quantitative test to determine whether Dachs contributes to morphogenesis: upon subtracting the contraction rate of any mutant condition abrogating Dachs function or polarization from the contraction rate of the wild-type condition, the resulting difference in contraction rate is expected to be aligned with the Dachs polarity pattern.

To investigate the role of Dachs polarization, we first quantified the Dachs polarity pattern (Fig 4A-B, green bars fig. S8). We then knocked-down Dachs or Ds function by expressing dachs $\left(d a c h s^{R N A i}\right)$ or $d s\left(d s^{R N A i}\right)$ hairpins during pupal development. We then averaged between 17:20 and 21:20 hAPF the map of differences of the local contraction rates between wild-type and $d a c h s^{R N A i}$ or between wild-type and $d s^{R N A i}$ pupae. We observed significant differences in the local contraction rates between wild-type and dachs ${ }^{R N A i}$ tissues or between 
wild-type and $d s^{R N A i}$ within the $\Lambda$-shaped domain of D:GFP polarization (blue bars in Fig. 4C,D, fig. S9A,B,C). The orientations of the significant differences were aligned with the local orientations of the D:GFP anisotropy distribution (Fig. 4C',D'). These results demonstrate that Dachs polarization regulates tissue morphogenesis by increasing the rate of contraction along its planar polarity lines.

We then studied how Dachs polarization controls the cell dynamics that makes up the local tissue contractions. Our analyses of cell division rate and orientation argue against a contribution of division orientation (fig. S10). We therefore quantified the respective contributions of cell rearrangements and cell shape changes to tissue contraction rates (fig. S11). Dachs polarization strongly correlates with cell rearrangements patterns both in magnitude and orientation (fig. S12A-C'), while it poorly correlates with cell shape changes (fig. S12D,D'). Accordingly, both the differences in tissue contraction rates between wildtype and $d a c h s^{R N A i}$, and between wild-type and $d s^{R N A i}$ were mainly associated with a decrease in the contribution of cell rearrangements to tissue contractions in the regions of strong Dachs polarization, and to a lesser extent, to cell shape changes (Fig. 4E'-F', fig. S12-13). Similar results were obtained in $f a t^{R N A i}$ and $f j^{U P}$ mutant conditions (fig. S14). Accordingly, $d s^{R N A i}, f j$, $f a t^{R N A i}$ and dachs $^{R N A i}$ pupae manifest similar defects in the adult scutellum shape (fig. S15). We conclude that the Fat/Ds/Fj PCP pathway regulates the local tissue contractions, mainly by promoting oriented cell rearrangements along the Dachs polarity lines.

Together these results have enabled us to advance the understanding of the Fat/Ds/Fj PCP pathway and define its morphogenetic role. We found that Ds polarization promotes Dachs polarization within a domain of the opposing tissue-wide $d s$ and $f j$ gradients. Their local polarization produces an anisotropic distribution of junction tensions, which increases the contraction rates along the lines of Ds and Dachs planar polarization to shape the epithelial tissue mainly by oriented cell rearrangements. More generally, our results indicate how global 
gene expression patterns can trigger local changes in mechanical cell properties to drive tissue morphogenesis. Furthermore by demonstrating that Ds itself is polarized, our study provides strong support for the long-standing hypothesis of a polarized activity of the $\mathrm{Fat} / \mathrm{Ds} / \mathrm{Fj}$ pathway $(8,9,11,13-16)$. Our results reveal that $\mathrm{Fat} / \mathrm{Ds} / \mathrm{Fj}$ pathway activity varies along the gradient of $d s$ and $f j$. This provides the opportunity to further understand how the shape and steepness of the $d s$ and $f j$ gradients can produce positional information within a tissue to regulate its development. Finally, given the multitude of cell shapes, cell sizes and division patterns present in the thorax epithelium as well as the repertoire of conserved signaling pathways known to regulate its development, future work in this tissue should reveal how multiple signaling pathways are integrated to regulate proliferation, planar polarization and morphogenesis.

\section{Acknowledgements}

We thank J. Axelrod, S. Blair, Y. Hong, K. Irvine, E. Martin-Blanco, M. Simon, D. Strutt, S. Tsukita, the Bloomington, TRiP and VDRC centers for reagents; M. Coppey, A. Houdusse, F. Molino, S. Ritzenthaler, F. Serman, J. Shi and the PICT-IBiSA@BDD for help; V. Hakim, J. Lopez-Gay, M. Labouesse and A-M. Lennon for comments. This work was supported by ARC-4830, ANR-MorphoDro, ERC-CePoDro grants, a FRM grant to I.B. and a NWORubicon grant to F.B.

\section{Figure Legends}

Figure 1. The Drosophila dorsal thorax as a system for morphogenesis. In all figures: macrochaetae, yellow circles; midline, cyan dashed line. (A) Dorsal thorax tissue labeled with E-Cad:GFP and imaged by confocal microscopy. The yellow shaded region outlines the scutellum. The scutum is outlined by a black line. The black box cut by the midline defines the two "hemi-scutellum" shown in following figures. (B-D) Maps of proliferation (B), apex 
area (C) and shape (D). (E) Local velocities represented as arrows, the gray shaded regions are described in the text. (F) Local deformation rates represented as ellipses. Scale bars: 100 $\mu \mathrm{m}, 910^{-2} \mu \mathrm{m} / \mathrm{min}$ (arrow in E), $2.410^{-3} \mathrm{~min}^{-1}$ (blue bar in $\mathrm{F}$ ),

Figure 2. Opposing gradients of $f j$ and $d s$ locally polarize Dachs and Ds in the scutellum. Panels show right-side hemi-scutellum. (A-A') Average patterns of Ds (A), fj-lacZ (A') and their quantification (A"). (B) Mean deformation rate $(n=5)$ between 17:20 and 21:20 hAPF. (C) D:GFP. (D-E) D:GFP in $d s^{R N A i}$ (D) and $f j{ }^{U P}$ (E) scutella. (F) Ds localization. Arrowheads indicate D:GFP (C) and Ds (F) polarity lines. Scale bars: $10 \mu \mathrm{m}, 10^{-3} \mathrm{~min}^{-1}$ (blue bar in B).

Figure 3. Ds and Dachs colocalize and Dachs polarization is associated with anisotropic tension. (A-B) Cells located in a region of Fj gradient expressing D:GFP and lacking D:GFP (D:GFP-, marked by the absence of D:GFP) (A), or overexpressing $d s$ ( $d s^{U P}$ cells marked by mRFP, not shown) (B) accumulate D:GFP and Ds at junctions facing the higher $\mathrm{Fj}$ concentration (green arrowheads), while they are absent at junctions facing the lower $\mathrm{Fj}$ concentration (red arrowheads). (C-C') D:GFP (C, C',) and Ds (C',C',) colocalize (arrowheads). (D) Anti-Flag Western blot of immunoprecipates from S2 cell lysates cells expressing Flag:Ds ${ }^{\text {intra }}$ and GFP; Flag:Ds ${ }^{\text {intra }}$; Flag:Ds ${ }^{\text {intra }}$ and D:GFP. MW (kDa) markers. (E) Graph of the mean tension measured as speed of vertex relaxation of junctions with high or low D:GFP. (F) Cell junctions with high or low D:GFP (arrows) 1560ms prior to ablation. (F') Images of Bazooka:mCherry prior to and following ablation. Arrowheads indicate the position of vertices. Scale bars: $10 \mu \mathrm{m}$.

Figure 4. The Dachs polarity lines promote local contractions mainly via oriented cell rearrangements. Averaged maps between 17:20 and 21:20hAPF. (A,B) Localization of 
D:GFP (A) and map of D:GFP magnitude and anisotropy (B) quantified by Fourier Transform

$(\mathrm{FT}, n=3) .\left(\mathbf{C}-\mathbf{F}^{\prime}\right)$ Maps of subtractions of $d a c h s^{R N A i}(n=5)$ from wt $(n=11)$ and of $d s^{R N A i}$ $(n=5)$ from wt $(n=11)$ for contraction rates $(\mathrm{C}, \mathrm{D})$ and cell rearrangements $(\mathrm{E}, \mathrm{F})$. Bars indicate local amplitudes and orientations of the differences of contraction rates or cell rearrangements. Maps of the alignment coefficient between the D:GFP FT and the differences in contraction rates ( $\left.C^{\prime}, D^{\prime}\right)$, or between the differences in contraction rates and cell rearrangements within the D:GFP FT pattern for wt and $d a c h s^{R N A i}\left(\mathrm{E}^{\prime}\right)$ and wt and $d s^{R N A i}\left(\mathrm{~F}^{\prime}\right)$. The local score of the alignment is color-coded ( $\mathrm{C}^{\prime}, \mathrm{D}^{\prime}$ in orange and $\mathrm{E}^{\prime}, \mathrm{F}^{\prime}$ in purple) and the average score $A$ is indicated. Significant data: green $(B)$, blue $(C, D)$ and red $(E, F)$ bars; other: gray bars $(B-F)$. Scale bars: $10 \mu \mathrm{m}, 5.510^{-4} \mathrm{~min}^{-1}$ (blue bars in C,D), $1 \mu \mathrm{m}^{2} \mathrm{~min}^{-1}$ (red bars in E,F).

\section{References}

1. L. V. Goodrich, D. Strutt, Development 138, 1877-92 (2011).

2. T. Lecuit, P. F. Lenne, E. Munro, Annu Rev Cell Dev Biol 27, 157-84 (2010).

3. F. A. Grusche, H. E. Richardson, K. F. Harvey, Curr Biol 20, R574-82 (2010).

4. P. A. Mahoney, U. Weber, P. Onofrechuk, H. Biessmann, et al., Cell 67, 853-68 (1991).

5. H. F. Clark, D. Brentrup, K. Schneitz, A. Bieber, et al., Genes Dev 9, 1530-42 (1995).

6. M. H. Brodsky, H. Steller, Dev Biol 173, 428-46 (1996).

7. H. O. Ishikawa, H. Takeuchi, R. S. Haltiwanger, K. D. Irvine, Science 321, 401-4 (2008).

8. M. A. Simon, A. Xu, H. O. Ishikawa, K. D. Irvine, Curr Biol 20, 811-7 (2010).

9. A. L. Brittle, A. Repiso, J. Casal, P. A. Lawrence, D. Strutt, Curr Biol 20, 803-10 (2010).

10. C. H. Yang, J. D. Axelrod, M. A. Simon, Cell 108, 675-88 (2002).

11. D. Ma, C. H. Yang, H. McNeill, M. A. Simon, J. D. Axelrod, Nature 421, 543-7 (2003).

12. H. Strutt, D. Strutt, Dev Cell 3, 851-63 (2002).

13. E. Cho, K. D. Irvine, Development 131, 4489-500 (2004).

14. H. Matakatsu, S. S. Blair, Development 131, 3785-94 (2004).

15. J. Casal, P. A. Lawrence, G. Struhl, Development 133, 4561-72 (2006). 
16. H. Matakatsu, S. S. Blair, Development 133, 2315-24 (2006).

17. Y. Mao, C. Rauskolb, E. Cho, W. L. Hu, et al., Development 133, 2539-51 (2006).

18. D. Rogulja, C. Rauskolb, K. D. Irvine, Dev Cell 15, 309-21 (2008).

19. H. Matakatsu, S. S. Blair, Curr Biol 18, 1390-5 (2008).

20. Y. Mao, A. L. Tournier, P. A. Bates, J. E. Gale, et al., Genes Dev 25, 131-6 (2011).

21. L. A. Baena-Lopez, A. Baonza, A. Garcia-Bellido, Curr Biol 15, 1640-4 (2005).

22. B. Aigouy, R. Farhadifar, D. B. Staple, A. Sagner, et al., Cell 142, 773-86 (2010).

23. Materials and methods are available as supporting material on Science Online.

24. G. Schwank, G. Tauriello, R. Yagi, E. Kranz, et al., Dev Cell 20, 123-30 (2011). 


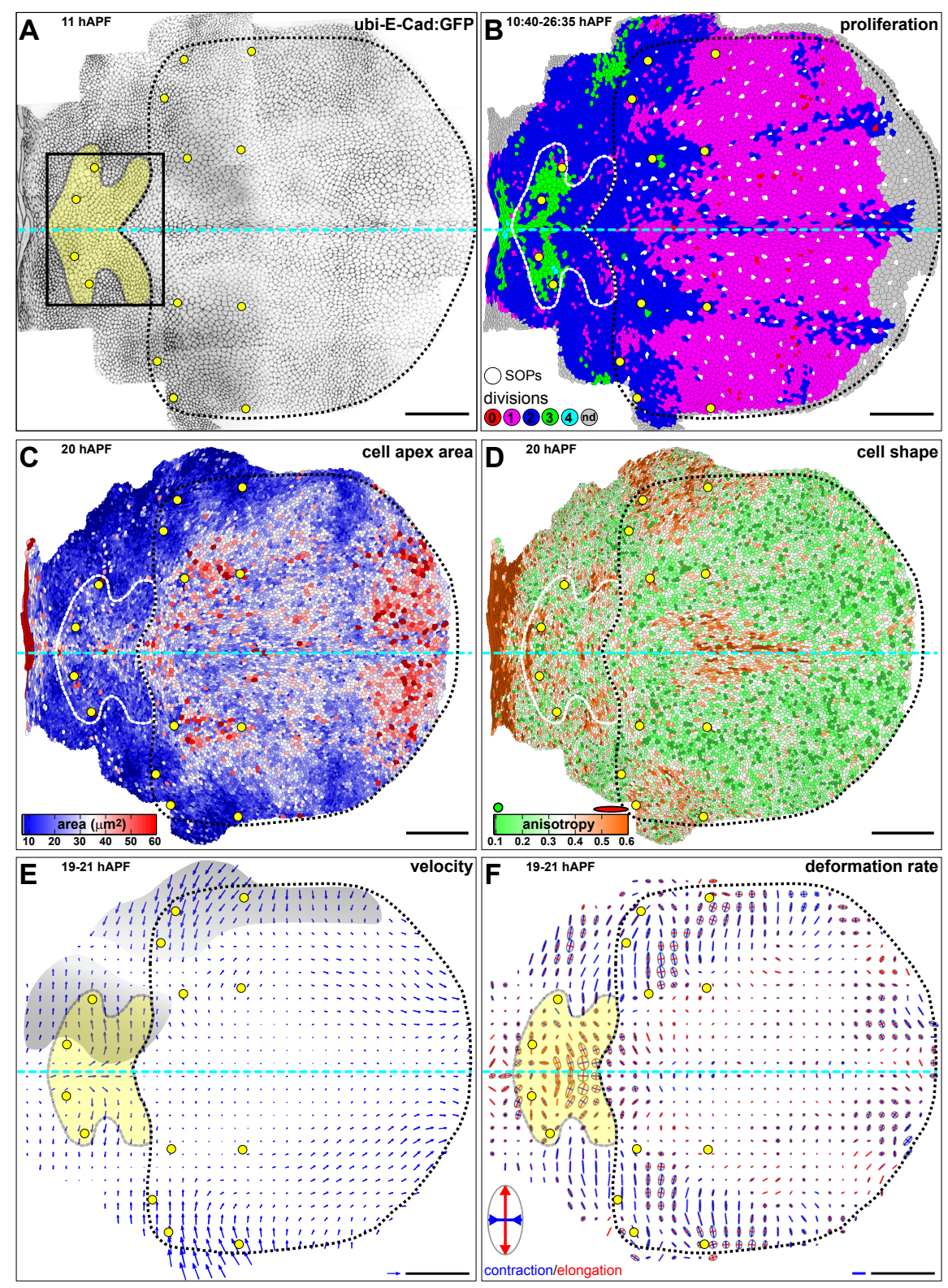




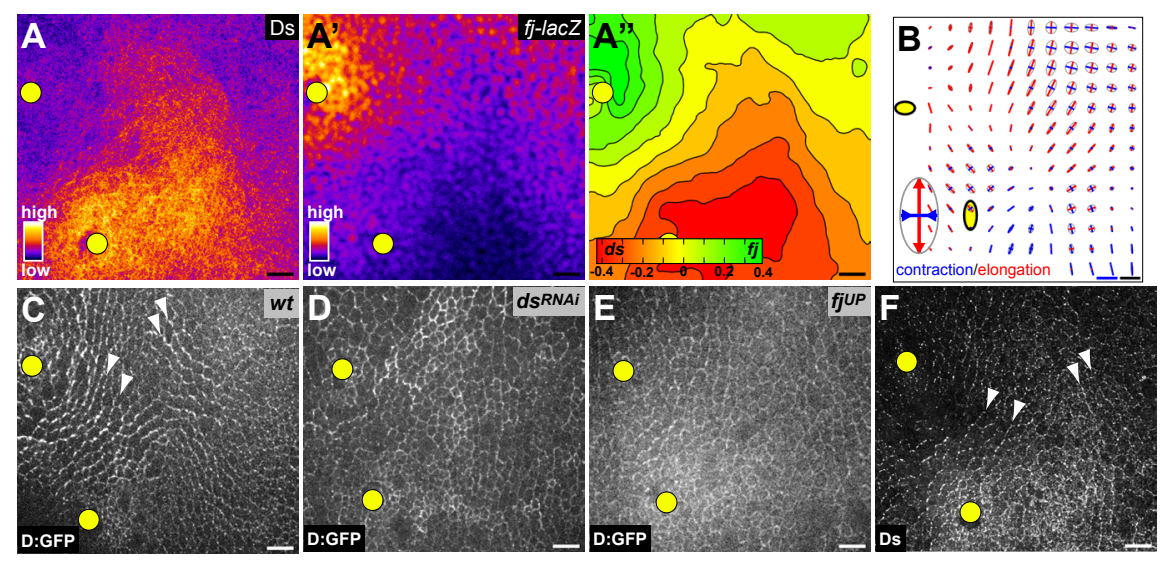

BOSVELD et al., FIGURE 2 


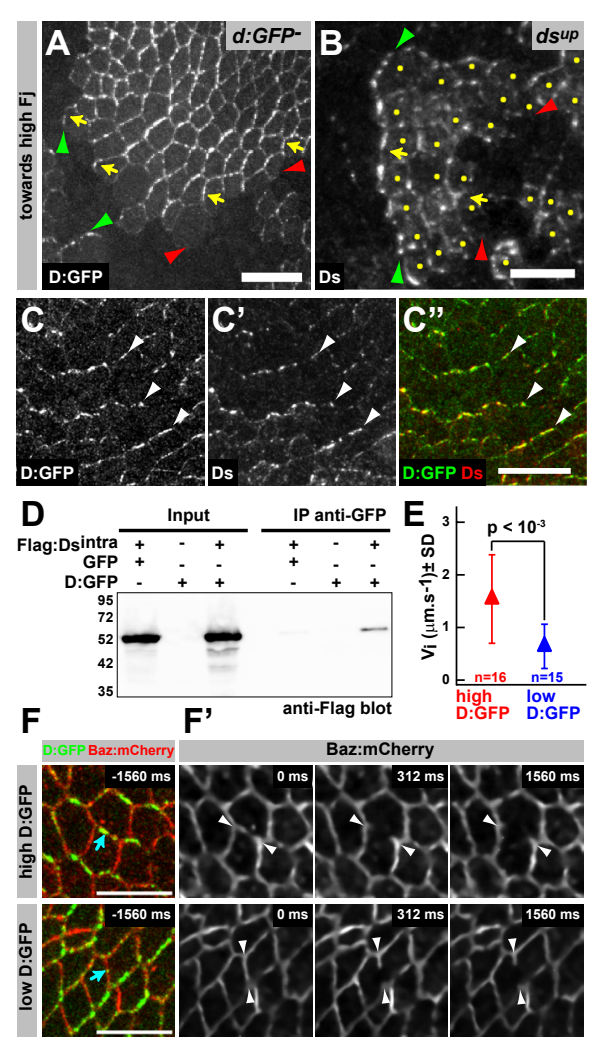



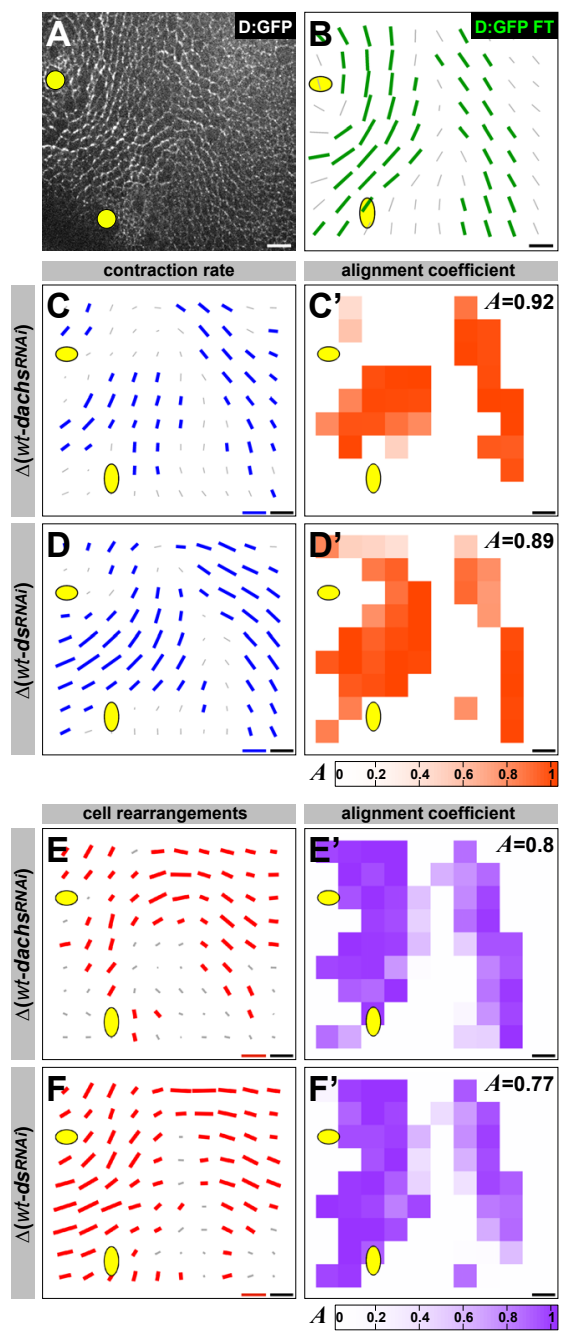


\section{Supporting Online Material (SOM)}

Mechanical control of tissue morphogenesis

by the Fat/Dachsous/Four-jointed planar cell polarity pathway

Floris Bosveld $^{1, *}$, Isabelle Bonnet ${ }^{1, *}$, Boris Guirao ${ }^{1, *}$, Sham Tlili ${ }^{1, \ddagger}$, Zhimin Wang ${ }^{1}$, Ambre Petitalot $^{1}$, Raphaël Marchand ${ }^{1}$, Pierre-Luc Bardet ${ }^{1}$, Philippe Marcq $^{2}$, François Graner ${ }^{1}$, Yohanns Bellaïche ${ }^{1, \sharp}$

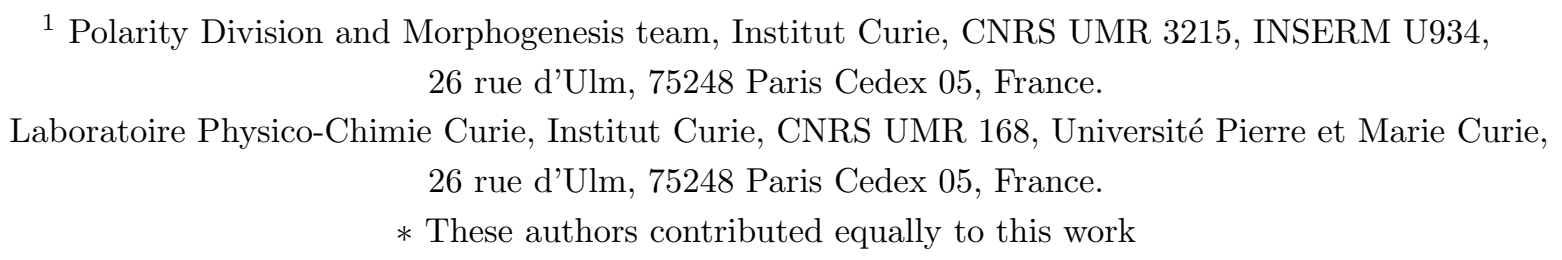

$\ddagger$ Present address: Matière et Systèmes Complexes, Université Paris Diderot, CNRS UMR 7057, 10 rue Alice Domon et Léonie Duquet, 75205 Paris Cedex 13, France

$\sharp$ Corresponding author: yohanns.bellaiche@curie.fr

\section{Contents}

1 Materials and methods $\mathbf{2}$

1.1 Fly stocks and genetics . . . . . . . . . . . . . . . . . . . . . . . 2

1.2 Molecular biology . . . . . . . . . . . . . . . . . . . . . . . . . . 2

1.3 Immunohistochemistry and fixed tissue imaging . . . . . . . . . . . . . . . . . . . . . . 3

$1.4 \mathrm{~S} 2$ cell culture and immunoprecipitation . . . . . . . . . . . . . . . . . . . . . . . 4

1.5 Live imaging . . . . . . . . . . . . . . . . . . . . . . . . . . . 4

1.6 Junction tension measurement by ablation . . . . . . . . . . . . . . . . . . . 5

2 Quantitative data analysis 6

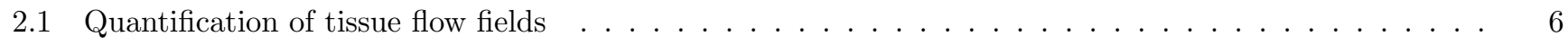

2.2 Comparing and averaging different movies . . . . . . . . . . . . . . . . . . . . . . 7

2.3 Quantification of D:GFP signal . . . . . . . . . . . . . . . . . . . . . . . .

3 Theoretical model 10

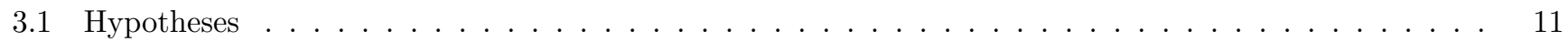

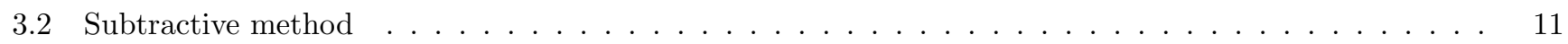

3.3 Predictions and experimental tests . . . . . . . . . . . . . . . . . . . . . 12

4 Segmented image analysis 13

4.1 Patterns of proliferation, cell apical area, and cell apical anisotropy . . . . . . . . . . . . . . 13

4.2 Cell shape changes and rearrangements . . . . . . . . . . . . . . . . . . . . . 14

5 Supporting figures and movies 15

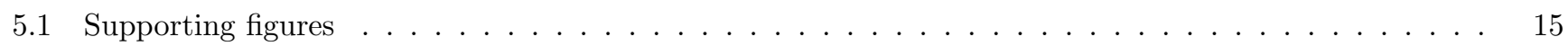

5.2 Supporting movie captions . . . . . . . . . . . . . . . . . . . . . . . . . . . . . . . . 


\section{Materials and methods}

\section{$1.1 \quad$ Fly stocks and genetics}

Fly stocks used in this study: UAS-ds (1), tub-FRT-GAL80-FRT-GAL4 UAS-mRFP (gift from E. Martin-Blanco), UAS-fj [1062] $U A S-f j{ }^{[1762]}(2), u b i-E-C a d: G F P(3), E-C a d: G F P(4), f j{ }^{[P 1]}$ (5), dachs ${ }^{[G C 13]}$ FRT40A (6), dachs ${ }^{[210]}$ FRT40A (6), fat ${ }^{[1]}$ FRT40A (6), fat ${ }^{[8]}$ dachs $^{[G C 13]}$ FRT40A (6), fat ${ }^{[G-r v]}$ FRT40A (7), sqh:mCherry (8); fly stocks from the Bloomington Stock Center: ds ${ }^{[05142]}$, act5C-GAL4 ${ }^{[25 F O 1]}$, tub-GAL4 ${ }^{[L L 7]}, t u b-G A L 80 t s^{[10]}$, tub-GAL80ts ${ }^{[2]}, U A S-f a t^{R N A i[T R i P . J F 03245]}, U A S-d s^{R N A i[T R i P . J F 02842]}, u b i-$ mRFP.nls FRT80B; fly stock from the VDRC Stock Center: UAS-dachs ${ }^{R N A i[v 12555]}$. A ubi-E-Cad:GFP insertion on the third chromosome was made by mobilizing the ubi-DE-Cad:GFP present on the second chromosome (3).

In all experiments the white pupa stage was set to 0 hour after pupa formation (hAPF), determined with $1 \mathrm{~h}$ precision. Mutant clones in the scutellum were generated using FLP/FRT mediated mitotic recombination $(9,10)$. Clones were induced in second instar larvae by heat shock $\left(20 \mathrm{~min}\right.$ at $\left.37^{\circ} \mathrm{C}\right)$. Clonal overexpression was achieved using a flip-out strategy (11) using the tub-FRT-GAL80-FRT-GAL4 transgene. Overexpression studies were carried out using the GAL4/UAS system (12).

Temporal control of gene function was achieved by using the temperature sensitive GAL80ts (13). Embryos and larvae were raised at $18^{\circ} \mathrm{C}$. Late third instar larvae were switched to $29^{\circ} \mathrm{C}$. After 24 to $30 \mathrm{~h}$, pupae were examined. Those which were timed as $11 \pm 1 \mathrm{hAPF}$ were mounted for live imaging at $29^{\circ} \mathrm{C}$. Some others were not mounted but kept further at $29^{\circ} \mathrm{C}$ until a total of $48 \mathrm{~h}$, transferred back to $18^{\circ} \mathrm{C}$, allowed to develop until adult stage, then photographed to quantify the shape of adult scutella.

\subsection{Molecular biology}

To create a D:GFP transgene under the control of its endogenous promoter, we used recombineering $(14,15)$ to introduce a GFP tag at the C-terminus of the dachs CDS present in BAC clone CH322-153C02 (BACPAC Resources Center). First a galK cassette, amplified with primers F (5'-CACAA GGGGCAGAGCTTCCACGTTCCCTTCCAGTTCATGACGCTCAGTAAACCTGTTGACAATTAATCA TCGGCA-3') and R (5'-CAGATGGAATGAATGGAATCATAACCAGTTTGTTTCCACCAGTAGTCCTT ATTCAGCACTGTCCTGCTCCTT-3') (underscored letters for galK sequences), was inserted via recombination at the C-terminus of the dachs CDS. After positive selection the galK cassette was replaced with the GFP tag, primers F (5'-CACAAGGGGCAGAGCTTCCACGTTCCCTTCCAGTTCATGACGCTCAGTA AAATGGTGAGCAAGGGCGAGGA-3') and R (5'-CAGATGGAATGAATGGAATCATAACCAGTTTGT TTCCACCAGTAGTCCTTACTTGTACAGCTCGTCCATGC-3') (underscored letters for GFP sequences) via recombination and negative selection for galK. The resulting attB-P[acman-d:GFP]-CmR-BW was integrated into the PBac $\{\mathrm{y}[+]$-attP-9A $\}$ VK00019 landing site at 68D2 creating $d: G F P^{[V K 19]}$. As a control the untagged attB-P[acman-dachs]-CmR-BW was also integrated at this site, dachs ${ }^{[V K 19]}$. Both $d: G F P^{[V K 19]}$ and $d a c h s^{[V K 19]}$ rescue morphological defects and the viability of dachs ${ }^{[G C 13]} /$ dachs $^{[210]}$ animals.

The ubi-Baz:mCherry transgene was constructed from pUASp-Baz-GFP (16). First the GFP was removed by 
digestion with NotI/XbaI and replaced with mCherry, amplified with primers F (5'-GACGGCGGCCGCAAT GGTGAGCAAGGGCGAG-3') and R (5'-CCGTCTAGATTACTTGTACAGCTCGTCC-3'), and digested with NotI/XbaI, resulting in pUASp-Baz-mCherry. Next, a KpnI-XbaI fragment containing Baz:mCherry was isolated from pUASp-Baz:mCherry and inserted, using identical restriction sites, between the ubiquitin promoter and hsp27 terminator present in a modified pCasper4 (http://flybase.org). A ubi-H2B:RFP transgene was constructed by removing an Acc65I-XbaI fragment from pUAST-H2B:RFP (17) containing H2B:RFP and inserting it using identical restriction sites between the ubiquitin promoter and hsp27 terminator present in a modified pCasper4. All transgeneses were performed by Bestgene.

\subsection{Immunohistochemistry and fixed tissue imaging}

For fixed tissue analyses, pupae were dissected at 18-20 hAPF as previously described (18). Primary antibodies used were: rat anti-Fj (1:250) (19), rat anti-Ds (1:2000) (20), rabbit anti-Ds (1:500) (21), rabbit anti- $\beta$-galactosidase (1:2000) (Cappel Laboratories), rabbit anti-GFP (1:2000) (Molecular Probes), rat anti-Fat (1:1000) (20), rat anti-DE-Cad (1:100) (DSHB, DCAD2). Secondary antibodies used were: Alexa488-conjugated goat-anti-rabbit IgG (Molecular Probes), Cy2-conjugated donkey-anti-mouse IgG (Jackson ImmunoResearch), Cy3-conjugated donkey-anti-mouse IgG (Jackson ImmunoResearch), Cy5-conjugated donkey-anti-rat IgG (Jackson ImmunoResearch). Images were collected with a confocal microscope (LSM 710 NLO, Carl Zeiss). All images represent maximum projections of a $Z$-stack unless otherwise specified.

LacZ enhancer trap lines were used to reveal the gene expression domains of $f j, f j{ }^{[P 1]}(5)$, and $d s, d s^{[05142]}(22)$. To quantify the opposing expression gradients of $f j$ and $d s$, heterozygous $f j{ }^{[P 1]}$ (5) pupae (20 hAPF) were fixed and labeled with antibodies against $\beta$-galactosidase to reveal the $f j$-lac $Z$ enhancer trap expression and antibodies against Ds to reveal the Ds protein gradient. Expression maps $(1880 \times 1770$ pixels $)$ were averaged over 7 different pupae aligned according to the positions of the macrochaetae which were determined by membrane staining with antibodies against E-Cadherin. Histograms of Fj and Ds images were equalized and normalized between 0 and 1 . Subtracting $\mathrm{Ds}$ from $\mathrm{Fj}$ resulted in an image in arbitrary unit in the range $[-1 ; 1]$. A Gaussian lowpass filter of size 150 pixels with standard deviation 50 pixels was applied and the resulting image represented as a filled contour plot where the $\Lambda$ shape appears as the orange band, of value $\sim-0.2$ (Fig. 2A").

From 29 hAPF onwards, the anterior-posterior boundary between the scutellum and the scutum can be easily distinguished since the scutellum and scutum are separated by rows of smaller cells. Therefore the position of the boundary can be precisely positioned on time-lapse movies by tracking back this group of smaller cells at earlier time-points. This reveals that the anterior-posterior boundary between the scutellum and the scutum has an indented shape from $11 \mathrm{hAPF}$ onwards; and that it is positioned between the group of large and small cells located between the posterior dorso-central and the anterior scutellar macrocheatae. On fixed tissue at $20 \mathrm{hAPF}$, the anterior-posterior boundary between the scutellum and the scutum can be approximately positioned using these criteria. 


\subsection{S2 cell culture and immunoprecipitation}

For cell culture experiments, a full length Dachs cDNA was Gateway cloned into pUbi-W-GFP (Drosophila Genomics Resource Center) creating ubi-D:GFP. The Ds intra cellular domain (aa 3115 to 3557) was inserted by Gateway cloning in pActin-Flag-W (Drosophila Genomics Resource Center) resulting in pActinFlag:Dsintra.

Drosophila S2 cells were maintained at $25^{\circ} \mathrm{C}$ in Schneider's Drosophila Medium (Gibco), containing $10 \%$ fœtal bovine serum inactivated at $65^{\circ} \mathrm{C}$, penicillin $(50 \mathrm{mg} / \mathrm{ml})$ and streptomycin $(50 \mathrm{mg} / \mathrm{ml})$ (Gibco).

$1.810^{7}$ cells were transfected with expression vectors by the Effecten method (Qiagen), and $72 \mathrm{~h}$ post transfection, cells were lysed at $4^{\circ} \mathrm{C}$ in lyses buffer: $50 \mathrm{mM}$ Tris- $\mathrm{HCl}(\mathrm{pH}$ 8), $150 \mathrm{mM} \mathrm{NaCl}, 1 \mathrm{mM}$ EDTA, $5 \mathrm{mM}$ glycophosphate, $0.1 \%$ Triton and protease inhibitor cocktail (Sigma). Total protein levels were determined by the Bradford method (Bio-Rad). For immunoprecipitation, lysates were incubated with rabbit-antieGFP antibodies coupled to protein A agarose beads. Cell lysates and immunoprecipitates were separated by SDS-PAGE, transferred to PVDF membranes (Millipore) and probed with mouse anti-Flag primary antibodies (Sigma) and subsequently with HRP-conjugated anti-mouse secondary antibodies (Jackson ImmunoResearch). Western blots were revealed by an enhanced chemiluminescence (ECL) detection system according to the manufacturer's instructions (Amersham Biosciences).

\subsection{Live imaging}

\subsubsection{Microscopy}

Pupae were prepared for live imaging as described in (23). Typically pupae were imaged for a period of 15-26 h, starting at 11-12 hAPF, with an inverted confocal spinning disk microscope (Nikon) equipped with a CoolSNAP HQ2 camera (Photometrics) and temperature control chamber, using Metamorph 7.5.6.0 (Molecular Devices) with autofocus.

Single position movies in the scutellum were acquired at either $25 \pm 1^{\circ} \mathrm{C}$ or $29 \pm 1^{\circ} \mathrm{C}$, with a 5 min temporal resolution (16-28 slices $Z$-stack, $0.5 \mu \mathrm{m} /$ slice for ubi-E-Cad:GFP, E-Cad:GFP and 20-50 slices $Z$-stack, $0.3 \mu \mathrm{m} /$ slice for D:GFP, ubi-Baz:mCherry). Acquiring 24 positions every 5 minutes $(Z$-stacks, 30 slices, $0.5 \mu \mathrm{m} /$ slice) yielded a tiling of the whole dorsal thorax (311 frames).

\subsubsection{Image treatment}

Movies of ubi-E-Cad:GFP and E-Cad:GFP are maximum projections of $Z$-stacks, D:GFP and Sqh:mCherry are average projections. They were obtained using a custom ImageJ routine (publicly available as the "Smart Projector" plugin) and have been used for tissue flow analysis. Multiple position movies were stitched using a customized version of the "StackInserter" ImageJ plugin. Image dynamics is defined as the decimal logarithm of the ratio of the largest to smallest scale, expressed in "decades" or "orders of magnitudes". Here, with 5 min temporal resolution over $26 \mathrm{~h}$ of development and $0.32 \mu \mathrm{m}$ spatial resolution over the $750 \times 700 \mu \mathrm{m}^{2}$ of the tissue, we follow the $\sim 10^{4}$ cells with a dynamics of $\sim 3$ decades in both space and time.

Movies of D:GFP and ubi-Baz:mCherry are average projections of $Z$-stacks. They were obtained using a 
custom Matlab routine that automatically detects the local $Z$ level showing the higher apical Baz:mCherry signal and removes the underlying background signal, then projects slices around this $Z$ level.

During the process of projection we kept records of slices containing the adherens junctions (i.e. the maximum intensity positions along the $Z$ axis. We thus established topographical maps $Z(X, Y)$ across the plane. The angle with the horizontal plane, $\theta_{\text {tilt }}$, is at most 0.01 radian over the scutellum, and reaches 0.02 radian only in the most lateral parts of the scutum. In the results presented here, the absolute correction due to this angle is thus negligible, $\left(1-\cos \theta_{t i l t}\right) \sim 10^{-4}$, and the curvature correction due to the spatial derivative of $\theta_{\text {tilt }}$ is negligible too.

\subsection{Junction tension measurement by ablation}

\subsubsection{Laser ablation of individual cell junctions}

Baz:mCherry and D:GFP scutellum tissues were imaged using a two-photon laser-scanning microscope (LSM 710 NLO, Carl Zeiss) in single-photon mode at a resolution of $100 \times 100$ pixels (pixel size $=0.18 \mu \mathrm{m}$ ) with a bidirectional scan lasting $\delta t=156 \mathrm{~ms}$. We used the $488 \mathrm{~nm}$ laser for D:GFP and the $561 \mathrm{~nm}$ one for Baz:mCherry. "Low" and "high" D:GFP cell junctions were individually ablated using a Ti:Sapphire laser (Mai Tai DeepSee, Spectra Physics) at $890 \mathrm{~nm}$ with $<100$ fs pulses with a $80 \mathrm{MHz}$ repetition rate. The laser power (typically $\sim 0.2 \mathrm{~W}$ at the back focal plane) was chosen to ablate a cell junction without creating cavitation (24).

Ablations at junctions between wt and $f a t^{[G-r v]}$ cells, and control ablations between differently marked wt cells, were performed in pupae expressing both Baz:mCherry and D:GFP. Ablations of junctions between wt and $f a t^{[8]}$ dachs ${ }^{[G C 13]}$ cells were performed in pupae expressing only Baz:mCherry. All ablations were performed in the scutum to avoid the endogenous polarization of Dachs.

\subsubsection{Quantification of junction tension}

Junction tension prior to ablation and relaxation speed of ablated junction vertices immediately after ablation are considered to be proportional (25-28). Baz:mCherry images were first denoised using Safir software (29) to improve the vertex localization accuracy. To determine the initial relaxation speed, the vertex-vertex distance $d_{\mathrm{vv}}$ of the pre-cut and post-cut ablated junction was measured manually (in blind procedure) using ImageJ at $n=2$ and $n=10$ frames after ablation.

Since the relaxation deviates from linearity already in the first 10 time steps, the measured relaxation speeds $V_{2}=\frac{d_{\mathrm{vv}}(2 \delta t)-d_{\mathrm{vv}}(0)}{2 \delta t}$ and $V_{10}=\frac{d_{\mathrm{vv}}(10 \delta t)-d_{\mathrm{vv}}(0)}{10 \delta t}$ are not equal. However, they are roughly proportional to each other, so that the conclusions obtained in Fig. 3E are robust with respect to the choice of $n$. Their amplitude does not correlate with the direction of the ablated junction prior to ablation (fig. S16). Conversely, as expected, the direction of the tension, and thus of the relaxation speed, fully correlates with the direction of the junction prior to ablation.

Statistical tests for data significance were performed using the Matlab's built-in function ttest2.m (twosample $t$-test). 


\section{Quantitative data analysis}

In our mechanical description, the local velocity describes the movements in the scutum and the scutellum. It includes all contributions to tissue morphogenesis regardless of their cellular origin, such as cell shape changes, rearrangements, divisions, apoptoses.

As described in fig. S17 or in ref. (30), a translation and a rotation (represented as an arrow and a circle, respectively) are movements which preserve the relative positions of cells. Change of size and/or shape thus results from velocity of tissue parts relative to each other: this is measured by the space variation of the velocity, i.e. velocity gradient, also called "deformation rate", and represented as an ellipse. Removing the size change from the deformation rate characterizes specifically the morphogenesis, i.e. change of shape: this is measured by the "contraction rate", and represented as a blue bar. We now develop these points one by one; for more details, refer to textbooks $(31,32)$.

\subsection{Quantification of tissue flow fields}

\subsubsection{Velocimetry}

The local tissue flow within scutum and scutellum tissues was quantified using movies of pupae expressing E-Cad:GFP or Baz:mCherry. For each movie, the flow field was obtained by image cross-correlation (IC) velocimetry along sequential images using customized Matlab routines based on the particle image velocimetry (33) toolbox, matpiv (http://folk.uio.no/jks/matpiv). Each image correlation window (hereafter "IC-box") was a $64 \times 64$ pixels square $\left(\sim 20 \times 20 \mu \mathrm{m}^{2}\right.$, pixel size $\left.0.32 \mu \mathrm{m}\right)$, had a $50 \%$ overlap with each of its neighbors, and typically contained a few tens of cells: this size corresponded to an optimum in the signal-to-noise ratio.

In each IC-box $(i, j)$, the velocity field $\mathbf{V}(i, j, t)$ was measured by correlating two successive images, at times $t$ and $t+\Delta t$ separated by $\Delta t=5 \mathrm{~min}$, and by performing a sliding average over $24 \Delta t=2 \mathrm{~h}$ to improve the signal to noise ratio without loss of information. The velocity field $\mathbf{V}$ was represented with arrows, expressed in $\mu \mathrm{m} \cdot \mathrm{min}^{-1}$. The tissue flow was identical when measured with E-Cad:GFP or Baz:mCherry. Within any single pupa where both hemi-scutella were imaged, the flow field appeared precisely symmetrical with respect to the midline: this acted as a control of the reliability of velocity measurements.

\subsubsection{Tissue flow representations}

Differentiating the velocity field yields the velocity gradient matrix, $\overline{\overline{\nabla \mathbf{V}}}$ :

$$
\overline{\overline{\nabla \mathbf{V}}}=\left(\begin{array}{ll}
\frac{\partial V_{x}}{\partial x} & \frac{\partial V_{y}}{\partial x} \\
\frac{\partial V_{x}}{\partial y} & \frac{\partial V_{y}}{\partial y}
\end{array}\right) .
$$

It is expressed in $\mathrm{min}^{-1}$; note that $10^{-3} \mathrm{~min}^{-1}=1 /(1000 \mathrm{~min})$ corresponds to a relative change of $1 \%$ in $10 \mathrm{~min}$.

To represent it, three quantities derived from it, also expressed in $\mathrm{min}^{-1}$, were computed and represented, as explained in fig. S17: the local deformation rate, contraction-elongation rate, and rotation rate. Mathematically, these quantities are defined as follows: 
- The deformation rate, $\overline{\overline{\nabla \mathbf{V}}}^{\text {sym }}$. It is the symmetrized part of the velocity gradient:

$$
\overline{\overline{\nabla \mathbf{V}}}^{\text {sym }}=\left(\begin{array}{cc}
\frac{\partial V_{x}}{\partial x} & \frac{1}{2}\left(\frac{\partial V_{x}}{\partial y}+\frac{\partial V_{y}}{\partial x}\right) \\
\frac{1}{2}\left(\frac{\partial V_{x}}{\partial y}+\frac{\partial V_{y}}{\partial x}\right) & \frac{\partial V_{y}}{\partial y}
\end{array}\right)=\left(\begin{array}{cc}
\nabla V_{x x}^{s y m} & \nabla V_{y x}^{s y m} \\
\nabla V_{x y}^{s y m} & \nabla V_{y y}^{s y m}
\end{array}\right) .
$$

Since $\overline{\overline{\nabla V}}^{\text {sym }}$ is a 2D symmetric matrix, it can be diagonalized and represented as an ellipse (fig. S17). which axis lengths are proportional to the two eigenvalues. A positive eigenvalue corresponds to a direction of elongation represented in red. Conversely, a negative eigenvalue corresponds to a direction of contraction represented in blue. Four possible cases are thus represented by four types of ellipses. (i) A positive isotropic dilation corresponds to two equal red bars. (ii) An ellipse with a red bar and a smaller blue bar is a traceless contraction-elongation plus a positive isotropic dilation. (iii) An ellipse with a red bar and a larger blue bar is a traceless contraction-elongation plus a negative isotropic dilation. (iv) A negative isotropic dilation corresponds to two equal blue bars.

- The contraction-elongation rate, $\overline{\overline{\nabla \mathbf{V}}}^{\text {dev }}$. It is obtained from the decomposition of $\overline{\overline{\nabla \mathbf{V}}}^{\text {sym }}$ into two contributions: its trace $\operatorname{Tr}\left(\overline{\overline{\nabla \mathbf{V}}}^{\text {sym }}\right)$, which reflects the overall isotropic dilation rate of apical area; and the traceless remaining part, also called the "deviator", $\overline{\overline{\nabla \mathbf{V}}}^{\text {dev }}$ :

$$
\overline{\overline{\nabla \mathbf{V}}}^{\text {sym }}=\frac{1}{2} \operatorname{Tr}\left(\overline{\overline{\nabla \mathbf{V}}}^{\text {sym }}\right) \overline{\overline{\mathbf{I}}}+\overline{\overline{\nabla \mathbf{V}}}^{\text {dev }}
$$

where $\overline{\overline{\mathbf{I}}}=\left(\begin{array}{ll}1 & 0 \\ 0 & 1\end{array}\right)$ is the identity matrix. Since $\overline{\overline{\nabla \mathbf{V}}}^{\text {dev }}$ is symmetric and traceless, it can be diagonalized with its two opposed eigenvalues $\pm \lambda$, which represent the rate of contraction in one direction and an equal rate of elongation in the perpendicular direction (and thus an effective change in tissue shape), at fixed apical area. Such traceless contraction-elongation corresponds to two orthogonal bars of equal lengths, one blue, one red. Since they are redundant, for clarity in all figures we plot only one: we choose the blue bar of length $|\lambda|$ in the direction of contraction (fig. S17).

- The rotation rate, $\omega$. It is the anti-symmetric part of $\overline{\overline{\nabla \mathbf{V}}}$ :

$$
\omega=\frac{1}{2}\left(\frac{\partial V_{y}}{\partial x}-\frac{\partial V_{x}}{\partial y}\right)
$$

This is a real number; it is represented as a circle, which diameter is the rotation rate $|\omega|$, and which color reflects its sign: red is for clockwise rotation and blue is for counter-clockwise (fig. S17).

\subsection{Comparing and averaging different movies}

We aimed at averaging different hemi-scutellum movies corresponding to the same genotype, or comparing movies corresponding to different genotypes. For that purpose, their common space and time coordinates were defined as follows.

\subsubsection{Space registration}

Each hemi-scutellum movie was oriented with the midline along the top horizontal side. Spatial landmarks (fig. S4A) were the positions of the two scutellar macrochaetae at 16:30 hAPF, when the sensory organ 
precursor (SOP) cells divide (34), and the results were independent of the choice of this reference time. The posterior scutellar macrochaeta defines the $X$ axis and the lateral scutellar macrochaeta sets the $Y$ axis. We find the positions of macrochaetae are reproducible up to $15 \mu \mathrm{m}$. The intersection of these $X$ and $Y$ axes defines the origin of the IC-grid, whereby $(0,0)$ is centered around one IC-box (green dashed square in fig. S4A). Each IC-box is then labelled by two integer numbers $(i, j)$ which define our two-dimensional space coordinates on a fixed square lattice (Eulerian representation). Averaging over space is implemented by averaging over $(i, j)$.

\subsubsection{Time registration}

While the hAPF was determined with $1 \mathrm{~h}$ absolute precision, the tissue rotation rate analysis provided a better relative precision that we used to synchronize the different movies, as follows. In the region of the tissue located near the origin of axes, we observed that the rotation rate (Eq. 4) systematically passed through a maximum during the development: this rotation peak could be used as a biological reference time. For that purpose, the rotation rate was measured and spatially integrated over a rectangular reference window (IC-boxes labelled $i=-2$ to 6 and $j=-2$ to 2 ). Plotting this average, $\bar{\omega}_{\text {ref window }}$, versus frame number yielded a bell-shaped curve (fig. S4B).

We applied a time translation to each movie so that these curves overlapped. We matched the portion of the curve which had the steepest slope (fig. S4B'): we thus used the time corresponding to $3 / 4$ of the peak value, in the ascent (rather than the maximum itself, which by definition had a vanishing slope). Its value, averaged over $n=11$ wt tissue movies was 18:40 hAPF. Hereafter, "hAPF" indicates the time after this temporal translation has been applied. For instance, after synchronization, the maximum of the contraction-elongation and rotation rates were consistently found at 19:20 and 19:40 hAPF, respectively. This determination reached $\mathrm{a} \pm 1$ interframe (i.e. $\pm 5 \mathrm{~min}$ ) relative precision in wt.

For experiments performed at $29^{\circ} \mathrm{C}$, the development is accelerated. This was taken into account by dividing time intervals by 0.9 (as determined with \pm 0.05 precision by the widening of the wt rotation peak). Mutant tissues were synchronized with the same procedure as the wt tissue, up to a 10 min precision (fig. S18).

\subsubsection{Ensemble average}

These spatial and temporal adjustments allowed us to assign a system of space-time coordinates $(i, j, t)$ common to any set of $N$ hemi-scutellum movies, from pupae with a given genotype $g$ (wt or mutant). We defined the ensemble averaged deformation rate:

$$
\overline{\overline{\nabla \mathbf{V}}}_{g}^{\text {sym }}(i, j, t)=\frac{1}{N} \sum_{n=1}^{N} \overline{\overline{\nabla \mathbf{V}}}_{g}^{\text {sym }}(i, j, t, n) .
$$

as well as its standard deviation $\delta_{g}\left[\overline{\overline{\nabla V}}^{\text {sym }}\right]$. Averages and standard deviations were measured over the space-time points $(i, j, t)$ for which experimental data were available in all movies after synchronization (intersection of the data). Since $\delta_{g}\left[\overline{\overline{\nabla V}}^{\text {sym }}\right]$ reflects our detection level, $\overline{\overline{\nabla V}}_{g}^{\text {sym }}(i, j, t)$ was considered as significant if $|\lambda|(i, j, t)$ was larger than $\delta_{g}\left[\overline{\overline{\nabla V}}^{s y m}\right](t)$ and plotted as blue bars, else as gray bars (Fig. 4C,D and figs. S9A-C, S13A,B, S14C,D). 


\subsection{Quantification of D:GFP signal}

Anisotropy of Dachs is due to a combination of cell shape anisotropy and of Dachs distribution around each given cell (section 2.3.4), which both contribute to stress (section 3.2.2). To quantify and average the Dachs signal distribution in wt hemi-scutella, we used movies of flies expressing both Baz:mCherry to label cell adherens junctions, and D:GFP. The tissue flow analysis was performed on Baz:mCherry in order to determine for all movies the spatio-temporal coordinates $(i, j, t)$ : these coordinates were then used for D:GFP signal quantification.

The 2D Fourier transform (FT) is a classical tool to extract the orientations of geometric patterns in an image (35). For example, the FT of an image of a regular pattern (such as a sinusoidal grid, see fig. S8A) leads to a FT whose signal is mainly in the direction orthogonal to the grid (fig. S8B-C) and can be represented as a green bar (fig. S8D). More generally, an image can be decomposed into a set of frequencies which reflect the existence, the spacing and direction of geometric patterns.

\subsubsection{Anisotropy of D:GFP}

To assess the anisotropy of D:GFP signal distribution in each IC-box (fig. S8E), its 2D discrete FT was performed using Matlab's built-in function $\mathrm{fft} 2 \mathrm{~m}$. In order to reduce spectral artifacts introduced by ICbox edges (35), the raw image was multiplied by a square cosine before computing its FT. To make the analysis robust with respect to possible translations of the D:GFP pattern within the IC-box, only the FT norm (and not its phase) was kept, and the same sliding average over $2 \mathrm{~h}$ as in flow analysis was performed to improve the signal to noise ratio. We determined its ensemble average over several movies, $\mathcal{F}(i, j, t)$ as well as the corresponding standard deviation $\delta[\mathcal{F}](t)$.

For each given $(i, j, t)$, the FT's norm was a function of the Fourier reciprocal space coordinates, $\mathcal{F}(\tilde{x}, \tilde{y}, t)$, corresponding to a distribution of gray levels. It was maximal at the center, $(\tilde{x}, \tilde{y})=(0,0)$ and decreased faster in the direction of the D:GFP pattern anisotropy than in the orthogonal direction (fig. S8F). This function was binarized by keeping only the pixels $(\tilde{x}, \tilde{y})$ having gray levels above the $80^{\text {th }}$ percentile. Such thresholding yielded a cluster of points displaying anisotropically distributed positions ( $\tilde{x}, \tilde{y}$ ) (fig. S8G). Its variance matrix $\left(\begin{array}{cc}\overline{\tilde{x}^{2}} & \overline{\tilde{y} \tilde{x}} \\ \overline{\tilde{x} \tilde{y}} & \tilde{\tilde{y}}^{2}\end{array}\right)$ was then computed. The traceless part of this matrix had two opposed eigenvalues $\pm \mu$ : here $|\mu|$ quantified the variance of D:GFP pattern anisotropy, so that $\sqrt{|\mu|}$ quantified its standard deviation. The eigenvector with a negative eigenvalue typically reflected the direction of the D:GFP pattern anisotropy.

\subsubsection{Amount of D:GFP}

In a semi-quantitative approach we constructed a number to quantify the observation that more contrasted parts of the image were the ones where D:GFP amount was larger. The local contrast $C(i, j, t)$ was thus defined as a relative intensity, as follows. We noted $P_{5}$ and $P_{95}$ the average intensity of the pixels below the $5^{\text {th }}$ percentile and above the $95^{\text {th }}$ percentile, respectively. For each IC-box at a given space-time point 
$(i, j, t)$, we then constructed the number $C(i, j, t)$ :

$$
C(i, j, t)=\frac{P_{95}(i, j, t)-P_{5}(i, j, t)}{P_{95}(i, j, t)} .
$$

$C(i, j, t)$ was found to range from 0.5 for lowest D:GFP signal regions up to 2 for highest D:GFP signal regions.

\subsubsection{Representation of D:GFP polarization: $\overline{\bar{Q}}$ matrix}

To combine the quantifications of anisotropy and amount of D:GFP signal, a matrix $\overline{\overline{\mathbf{Q}}}(i, j, t)$ was built as follows. Its eigenvectors were those defined in section 2.3.1: they were typically parallel and orthogonal to the main anisotropy direction of the local D:GFP pattern. Its eigenvalues were defined as $\pm C(i, j, t) \sqrt{|\mu|(i, j, t)}$, where $\sqrt{|\mu|}$ quantified the standard deviation of the D:GFP pattern anisotropy, and the weight $C$ quantified the amount of D:GFP (section 2.3.2). We ensemble averaged $\overline{\overline{\mathbf{Q}}}$ over $n=3$ movies.

A green bar of length $C(i, j, t) \sqrt{|\mu|(i, j, t)}$ was plotted in the direction of the negative eigenvalue of $\overline{\overline{\mathbf{Q}}}$ (fig. $\mathrm{S} 8 \mathrm{H})$. The bar length and direction reflected the visible characteristics of the D:GFP pattern, which could be averaged. More precisely, we considered $\overline{\overline{\mathbf{Q}}}(i, j, t)$ as significant if $\sqrt{|\mu|(i, j, t)}$ was larger than $\delta[\mathcal{F}](t)$.

\subsubsection{Comparisons of D:GFP, Sqh:mCherry and E-Cad:GFP anisotropies}

As already mentioned, anisotropy of Dachs is due to a combination of cell shape anisotropy and of Dachs distribution around each given cell. To disentangle these effects, we measured the anisotropy $\sqrt{|\mu|(i, j, t)}$ of D:GFP, Sqh:mCherry and of E-Cad:GFP in $n=3$ movies at $25^{\circ}$ for each condition (fig. S7G,G',H). The procedure was the same as in section 2.3.1, with the $70^{\text {th }}$ percentile instead of the $80^{\text {th }}$, to adapt to the movies quality.

The Sqh:mCherry and E-Cad:GFP patterns are similar and reflect cell elongations (compare figs. S7G' and H). Almost everywhere, they are not significantly different from zero. They are just below or just above the threshold only at few places where cells are much more elongated.

\section{Theoretical model}

We sought to investigate whether there was a connection between: (i) the observed D:GFP polarization; (ii) the Dachs-related contribution to mechanical stress; and (iii) the Dachs-related contribution to tissue flow. For that purpose, we have developed a physical model of the relation between stress and flow. This analysis led to the identification of relevant quantities: first, a matrix characterizing the contraction rate; second, a semi-quantitative estimate of stress based on a matrix characterizing Dachs polarization. Furthermore, the model predicted that these matrices were linked and were locally proportional to each other, which was experimentally tested.

In short, the prediction of our physical model is: if D:GFP imposed a stress pattern to the tissue then for every mutant affecting Dachs polarization, we should observe that the difference in tissue contraction between this mutant and the wt condition should correlate with the D:GFP polarization pattern. Combining 
physical modeling and subtractive approach thus extracts and evidences the contribution of a given individual pathway within an elaborated morphogenetic movement. It thus links genetics and mechanics in the context of tissue morphogenesis. We explicit the model in the context of the dachs ${ }^{R N A i}$ mutant condition. Similar conclusions can be reached using the $d s^{R N A i}, f a t^{R N A i}$ or $f j{ }^{U P}$ mutant conditions which affect the pattern of Dachs polarization.

\subsection{Hypotheses}

We treated the flow in two dimensions because: (i) the dorsal thorax epithelium is a quasi-2D monolayered tissue with negligible curvature; (ii) the network of the adherens junction belts which we image, and which enables long-range transmission of in-plane stresses within tissues (36), is only a few $\mu \mathrm{m}$ thick; and (iii) the visible in-plane components of the tissue flow velocity likely dominate its possible out-of-plane components.

Developing tissues have been observed to display simultaneously liquid-like behaviours (flow at large scale), and solid behaviours (elastic deformations of cells, irreversible plastic shape changes) (37-39). However, under the effect of active cell shape fluctuations (37) or cell divisions (40), the liquid behaviour dominates $(37,39,41)$ at times larger than the characteristic visco-elastic time, which is reported to be of order of minutes $(37,39,42)$. Here, morphogenetic movements had a time scale of the order of 2 hours (velocity gradients at most of a few hundredths of $\mathrm{min}^{-1}$ ). We then safely assumed that the dominant contribution to mechanical stress matrix $\overline{\bar{\sigma}}$ was viscous $(37,40)$ and wrote $(43)$ :

$$
\overline{\bar{\sigma}}=\left[-p+2 \eta_{\text {comp }} \operatorname{Tr}\left(\overline{\overline{\nabla \mathbf{V}}}^{\text {sym }}\right)\right] \overline{\overline{\mathbf{I}}}+2 \eta_{\text {eff }} \overline{\overline{\nabla \mathbf{V}}}^{\text {dev }} .
$$

Here $p$ is the pressure; $\eta_{\text {comp }}$ a compressibility dissipation, which includes several contributions; $\eta_{\text {eff }}$ is the usual hydrodynamic viscosity: the subscript "eff" recalls that it is an effective viscosity which not only includes the contribution of cytoplasmic viscosity, but also active contributions from the cytoskeleton, or from neighbor rearrangements (37). The deformation rate $\overline{\overline{\nabla \mathbf{V}}}^{\text {sym }}$ and the contraction-elongation rate $\overline{\overline{\nabla \mathbf{V}}}^{d e v}$ are defined in Eqs. (2,3); the traceless part of the stress matrix $\overline{\bar{\sigma}}^{d e v}$ is defined as in Eq. 3:

$$
\overline{\bar{\sigma}}^{d e v}=\overline{\bar{\sigma}}-\frac{1}{2} \operatorname{Tr}(\overline{\bar{\sigma}}) \overline{\overline{\mathbf{I}}}=2 \eta_{\text {eff }} \overline{\overline{\nabla \mathbf{V}}}^{d e v} .
$$

Eq. 8, which is independent from the trace, reflects the contraction-elongation part of the flow, involved in changes of shape (i.e. morphogenetic movements), and of interest in what follows.

\subsection{Subtractive method}

\subsubsection{Modeling}

Since Eq. 8 is linear, one can use it to write the stress in presence of Dachs, that is, in the wt context, $\overline{\bar{\sigma}}_{\mathrm{wt}}^{d e v}$, minus the stress in absence of Dachs, $\overline{\bar{\sigma}}_{\text {dachs }}^{\text {dev }}{ }^{R A i}$ :

$$
\overline{\bar{\sigma}}_{\mathrm{wt}}^{d e v}-\overline{\bar{\sigma}}_{d a c h s^{R N A i}}^{d e v}=2 \eta_{\mathrm{eff}}\left(\overline{\bar{\nabla}}_{\mathrm{wt}}^{d e v}-\overline{\overline{\nabla \mathbf{V}}}_{\text {dachs }}^{\text {dev }}{ }^{R N A i}\right) .
$$

The left and right hand sides of Eq. 9 reflect the specific contribution of Dachs to the stress and to the flow, respectively. We introduce the compact notations:

$$
\begin{aligned}
\overline{\bar{\sigma}}_{\text {Dachs }} & =\overline{\bar{\sigma}}_{\mathrm{wt}}^{\text {dev }}-\overline{\bar{\sigma}}_{\text {dachs }}^{\text {dev }}{ }_{\text {dAi }}, \\
\overline{\overline{\nabla \mathbf{V}}}_{\text {Dachs }} & =\overline{\bar{\nabla}}_{\mathrm{wt}}^{\text {dev }}-\overline{\bar{\nabla}}_{\text {dachs }}^{\text {dev }}
\end{aligned}
$$


In order to avoid confusion between the left and right hand sides of Eqs. 10, and since we only consider the traceless parts of matrices in what follows, we have dropped the dev superscript. Using these notations, Eq. 9 reads:

$$
\overline{\bar{\sigma}}_{\text {Dachs }}=2 \eta_{\text {eff }} \overline{\overline{\nabla V}}_{\text {Dachs }}
$$

\subsubsection{Measurable quantities}

Eq. 11 establishes a proportionality relation between two quantities which can be measured in experiments. In the right hand side, $\bar{\nabla}_{\text {Dachs }}$ can be directly measured. In the left hand side, since Dachs-rich cell junctions were under a larger tension and were apparently distributed anisotropically, one could expect that Dachs had an anisotropic contribution to $\overline{\bar{\sigma}}_{\text {Dachs }}$ : it was stronger in the direction of strong Dachs intensity than in the perpendicular direction. This contribution to stress could be estimated by measuring both the length and tension of each cell-cell junction (44). Here the tension measurement required the quantitative determination of: (i) D:GFP amount based on fluorescent intensity, and (ii) of the relation between D:GFP quantity and tension. Both (i) and (ii) were beyond the scope of this study.

Instead, we used the matrix $\overline{\overline{\mathbf{Q}}}$ built in section S2.3.3 as a semi-quantitative measurement of Dachs distribution, combining both cell shape anisotropy and Dachs distribution around each given cell. In the case where Dachs was absent or isotropically distributed, the FT signal was zero or isotropic, and $\overline{\overline{\mathbf{Q}}}$ was zero by construction. Thus $\overline{\bar{\sigma}}_{\text {Dachs }}$ and $\overline{\overline{\mathbf{Q}}}$ vanished simultaneously; in a linear approximation one could thus reasonably expect that they were proportional to each other:

$$
\overline{\overline{\mathbf{Q}}}=\operatorname{cst} \times \overline{\bar{\sigma}}_{\text {Dachs }}
$$

\subsection{Predictions and experimental tests}

\subsubsection{Predictions regarding experiments}

Combining Eqs. 11-12 immediately yields a testable relation between two operationally measurable quantities:

$$
\overline{\overline{\mathbf{Q}}}=\operatorname{cst} \times \overline{\overline{\nabla \mathbf{V}}}_{\text {Dachs }} .
$$

Eq. 13 is our main prediction. It states that the distribution of Dachs planar polarization, measured by D:GFP FT, correlates with the local subtraction of dachs ${ }^{R N A i}$ from wt contraction-elongation rates.

Eq. 13 can thus be tested by comparing the patterns of $\overline{\overline{\mathbf{Q}}}$ and $\overline{\overline{\nabla \mathbf{V}}}$ Dachs in the scutellum. If there was a direct or indirect causal relation between $\overline{\overline{\mathbf{Q}}}$ and $\overline{\overline{\nabla V}}_{\text {Dachs }}$, and if this relation was indeed local, then patterns of both matrix fields should look similar.

\subsubsection{Tests}

As a first test, one could perform a direct visual comparison of data. Indeed, after averaging over the time interval 17:20-21:20 hAPF where contraction-elongation was the largest, similar qualitative features were observed on the zones where the matrices $\overline{\overline{\mathbf{Q}}}$ or $\overline{\bar{\nabla}} \overline{\mathbf{V}}_{\text {Dachs }}$ were significant. The position, size and shape of these zones were similar on both patterns. Within these zones, the orientation of $\overline{\overline{\mathbf{Q}}}$ and $\overline{\overline{\nabla \mathbf{V}}}$ Dachs appeared similar (Fig. 4B,C). 
To make this test more quantitative, we quantified the local alignment between the blue bars of the contraction rate and the green bars of D:GFP patterns. These bars made a relative angle noted $\alpha(i, j)$. Since bars were not oriented, $\alpha$ was defined modulo $\pi$, and the local alignment could be characterized by:

$$
A(i, j)=\cos ^{2} \alpha(i, j) \text {. }
$$

Averaging Eq. 14 over all IC-boxes where both $\overline{\overline{\nabla V}}_{\text {Dachs }}$ and $\overline{\overline{\mathbf{Q}}}$ were significant yielded its spatial average, $\bar{A}$ (indicated on Fig. 4C'):

$$
\bar{A}=\frac{\sum_{i, j} p(i, j) \cos ^{2} \alpha(i, j)}{\sum_{i, j} p(i, j)} .
$$

Here the weights include the norms of both matrix fields, $p(i, j)=\left|\overline{\overline{\nabla V}}_{\text {Dachs }}(i, j)\right| \times|\overline{\overline{\mathbf{Q}}}(i, j)|$, averaged over 17:20-21:20 hAPF. $\bar{A}$ reflects the overall alignment of $\overline{\mathbf{Q}}$ and $\overline{\overline{\nabla V}}_{\text {Dachs. If the bars of }} \bar{\nabla}_{\text {Dachs }}$ and $\overline{\overline{\mathbf{Q}}}$ remained always perfectly orthogonal or colinear, then $\bar{A}=0$ or 1 , respectively. In between, $\bar{A}$ varied between 0 and 1. In the case where $\overline{\overline{\nabla \mathbf{V}}}_{\text {Dachs }}$ and $\overline{\overline{\mathbf{Q}}}$ were not correlated, their relative angle $\alpha$ varied randomly, and $\bar{A}=0.5 \pm 0.01$, where we have determined the standard deviation by running fifty simulations of random angles, with same sample size as in our experiments. The alignment coefficient $\bar{A}$ of $\overline{\overline{\nabla V}}_{\text {Dachs }}$ (or of any other matrix) with the FT pattern $\overline{\overline{\mathbf{Q}}}$ was coded in orange, from 0 for fully anti-correlated, to 1 for fully correlated, through 0.5 for non correlated (Fig. 4C'D', fig. S12C'D', fig. S14C'D').

\section{Segmented image analysis}

To reinforce the multiscale description of morphogenetic movements, from cell level to tissue level, we performed detailed cell-level analysis. Images were denoised with the Safir software (29). Cell contours were determined and individual cells were identified using a standard watershed algorithm, followed by several rounds of manual corrections. Around 2.8 million cells (8.4 million cell-cell junctions) were segmented. We estimate the error rate to be below $10^{-4}$, which is sufficient for the analyses presented here.

\subsection{Patterns of proliferation, cell apical area, and cell apical anisotropy}

Four wt scutellum movies at $25^{\circ} \mathrm{C}$ were segmented over 156 frames. An in-house automatic software (Matlab) tracked segmented cells through all images. It yielded maps of cell centroid displacements (fig. S10C), cell lineages and lists of divisions (fig. S10B,C and Movie S2C,C'), and lists of apoptoses (Movie S2C,C').

We detected 1591 divisions with an error rate estimated below 1\%. The wt movies were synchronized using IC analysis (section 2.2.2) to obtain an ensemble averaged number of divisions plotted versus hAPF with again a sliding average over $24 \Delta t=2 \mathrm{~h}$. A division angle $\Theta$ was defined as the angle between AP axis and the line connecting the centroids of the daughter cells in their first image of appearance. Division angles were scored for $\Theta$ in $\left[0^{\circ}, 180^{\circ}\right]$. For clarity, scores were represented in polar histograms ("rose plots") around the full circle by displaying both $\Theta$ and $\Theta+180^{\circ}$ (respectively dark blue and blue in fig. S10B).

For whole thorax movies (Movie S1), each image contained in average around $10^{4}$ cells and parts of the movies were segmented. Measurements performed on single images yielded maps of cell apical area and anisotropy (Fig. 1C-D and fig. S1C,D). The cell division pattern reported in Fig. 1B was determined 
manually by visual tracking of cell divisions through all images. Cells in the anteriormost part get out of focus due to tissue flow and elongation, so that we cannot determine the total number of cell divisions that they undergo (gray cells in Fig. 1B).

\subsection{Cell shape changes and rearrangements}

To quantify cell shape changes, Rauzi et al. (see the figs. 5a and S4 of ref. (26)) used a matrix called "texture" as defined in Eq. 6 of ref. (45). It is based on the links connecting each cell center with the centers of its neighbors: it expresses, in $\mu \mathrm{m}^{2}$, the variance of link length in each direction. Separately, rearrangements were counted and quantified as integer numbers. Similarly, Aigouy et al. (46) used a former version of the texture as defined in the Eq. 1 of ref. (47), which expressed the variance of the cell junctions in each direction. More precisely, they call "cell elongation" (defined in Eq. S25 of ref. (46)) the traceless part of the texture. Again, rearrangements were quantified separately, and as integer numbers (see Fig. 4 of ref. (46)). Note that in a rearrangement a cell junction vanishes and a new junction is created. It is sometimes followed by the opposite rearrangement, where the new junction vanishes and the original one is re-created. Since counting rearrangements as numbers does not take into account the directions of junctions, a rearrangement plus its opposite are counted as $1+1=2$ rearrangements, although their total effect does not contribute to tissue morphogenesis. Conversely, Butler et al. (48) used their own method (defined in ref. (30)). They computed the contribution of cell shape change to flow. They then subtracted the cell shape change rate from the tissue shape change rate. They thus indirectly inferred the correct contribution of rearrangements to morphogenesis as matrices, i.e. including their amplitude but also their direction.

Here, to characterize cell dynamics in term of cell shape changes and cell rearrangements, we apply to tissue morphogenesis a formalism based on texture, validated on foam dynamics (45). Overall, $10 \mathrm{wt}, 5$ dachs ${ }^{R N A i}$, $5 d s^{R N A i}, 4 f_{a t}{ }^{R N A i}$ and $2 f j{ }^{U P}$ movies at $29^{\circ}$ were segmented and analyzed in the interval 17:20 - 21:20 hAPF. The texture (Eq. 6 of ref. (45)) of all cells located in the field of view was measured from the beginning to the end of the 4 hours under consideration. The cumulative variation of texture over these 4 hours was calculated in each IC-box.

Cell shape changes were defined as the rate of texture variation, expressed in $\mu \mathrm{m}^{2} \mathrm{~min}^{-1}$ (Eq. C3 in appendix of ref. (45)). They were measured as matrices, in order to retain information regarding their direction and anisotropy, relevant to characterize contributions to tissue shape change. In what follows we do not use their trace, related to changes in cell sizes. They were represented as cyan bars along the direction of cell contractions (fig. S11A).

Cell rearrangements too were directly measured as matrices in the same formalism and expressed in the same units, namely $\mu \mathrm{m}^{2} \mathrm{~min}^{-1}$. They were measured as follows (Eq. 11 of ref. (45)): links which disappear (yellow lines in Movie S2C,C') contribute negatively, while links which appear (red lines in Movie S2C,C') contribute positively. They were represented as red bars along the direction of center-center links which appear, thus in the direction of cell-cell junctions that disappear (fig. S11B). If two cells transiently lose their junction, then regain it, their link disappears then re-appears, and the total contribution of such backand-forth rearrangement to the rearrangement matrix is close to zero, as it should be. When a four-fold vertex was detected during a rearrangement, corresponding links (shown in lighter colours in Movie S2C,C') 
were counted with a weight $1 / 2$ so that the total contribution of the corresponding rearrangement was independent of the detection of the four-fold vertex.

Using movie registration in time and space, measurements were averaged over several movies, thus providing a good signal to noise ratio. This enabled us to use our subtractive method (section 3.2.1) to calculate the local differences in cell rearrangements and cell shape changes in wt and mutant conditions that contribute to the differences in local tissue contraction rates. This led to the determination of the specific Dachs polarization contributions to cell rearrangements or to cell shape changes. In turn, these contributions were correlated with the differences in tissue contraction rates over regions of strong D:GFP polarization. The mutual alignment coefficient $\bar{A}$ of cell shape changes and contraction rate, or rearrangement rate and contraction rate, was plotted in the spirit of section 3.3.2 at places where the FT pattern $\overline{\overline{\mathbf{Q}}}$ was significant (purple in Fig. 4E'F', fig. S13A"B", fig. S14E'-H').

\section{$5 \quad$ Supporting figures and movies}

\subsection{Supporting figures}

In all figures, the positions of the macrochaetae and of the midline are indicated by yellow circles and by a cyan dashed line, respectively. Data on flow, FT and division are averaged over $64 \times 64$ pixels squares $\left(\sim 20 \times 20 \mu \mathrm{m}^{2}\right)$ and 24 interframes $(2 \mathrm{~h})$. Such space and time intervals are large enough to yield a signal to noise ratio suitable for the analyses presented here, and small enough to observe relevant details in morphogenetic movements.

Figure S1. Metamorphosis of the Drosophila dorsal thorax. The visible symmetry of the patterns with respect to the midline underlines that they are finely regulated at the scale of 10-20 cell clusters rather than at the individual cell level. Using Gaussian filters of increasing sizes confirms that these patterns become completely symmetric when blurred above 10-20 cell sizes.

(A) Drosophila adult dorsal thorax. The visible part of the adult scutellum is shaded in yellow and the scutum is outlined by a white dashed line. (B) Dorsal view of the Drosophila pupa without pupal case, here at $14 \mathrm{hAPF}$. The white box indicates the position of the thorax. (C-D) Cell-level measurements at 11, 20, 29 and $35 \mathrm{hAPF}$ during morphogenesis of the pupa dorsal thorax. (C) Cell apex area, in color code scale from blue $\left(10 \mu \mathrm{m}^{2}\right)$ to red $\left(60 \mu \mathrm{m}^{2}\right)$. (D) Cell shape anisotropy, defined as one minus the ratio between the minor to major axes of the best fitting ellipse, from green (0.1) to brown (0.6), see insets below the color bar. The scutellum is outlined by a white dashed line, the scutum by a black one. Scale bars: $250 \mu \mathrm{m}(\mathrm{A}$, B), $100 \mu \mathrm{m}(\mathrm{C}, \mathrm{D})$. 


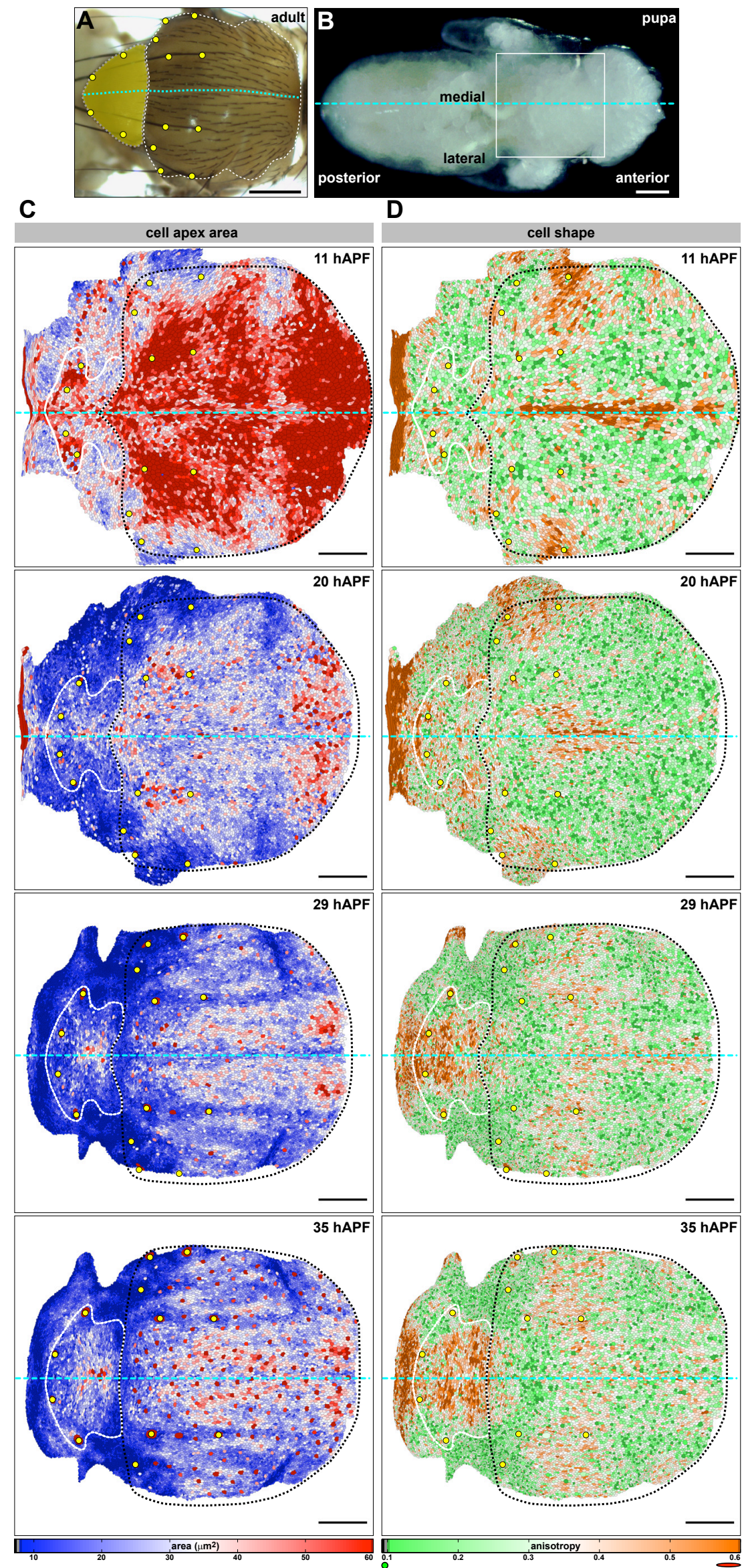



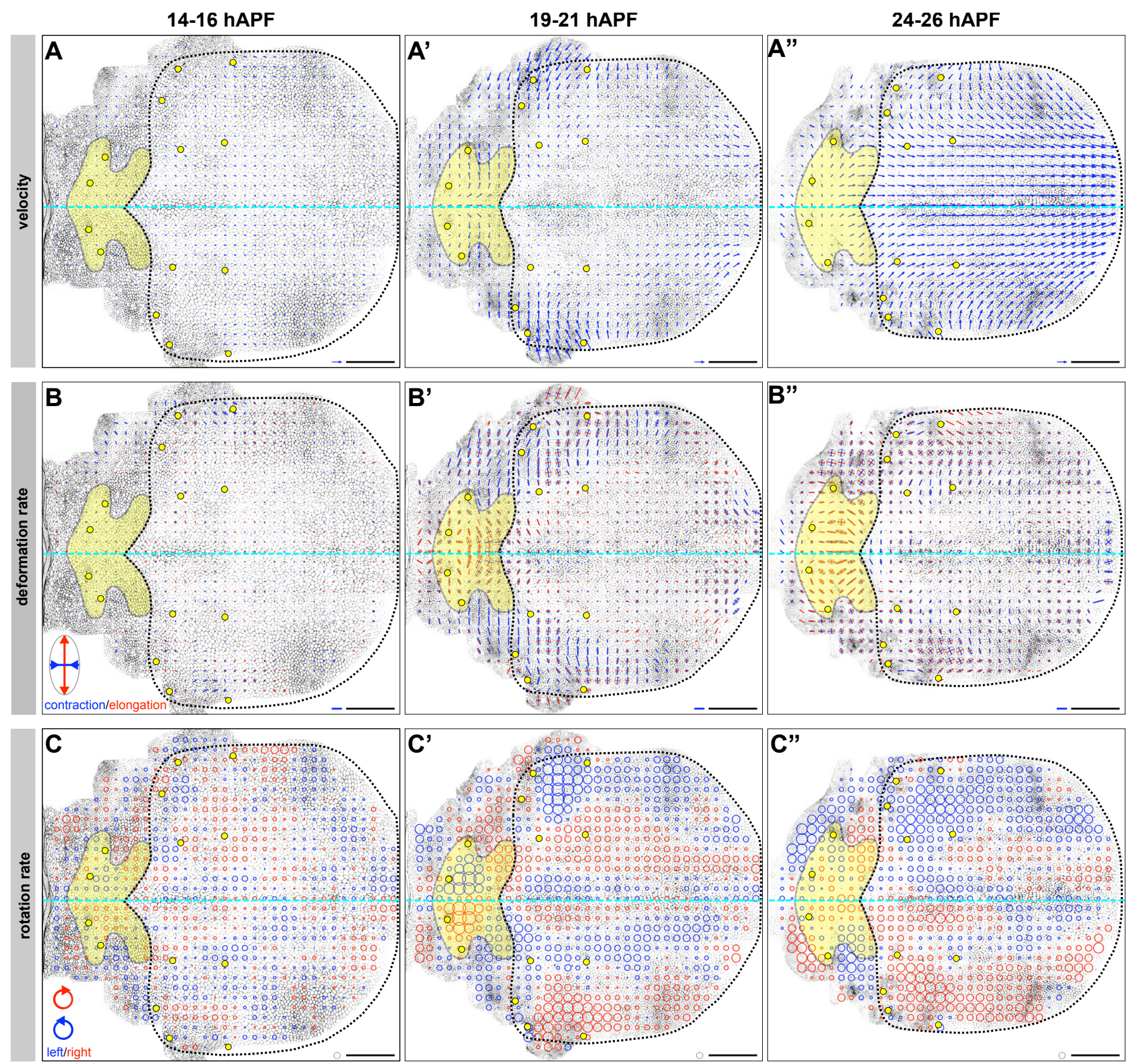

Figure S2. Tissue dynamics in the pupa dorsal thorax during metamorphosis. These maps are representative of the three main phases of dorsal thorax development. (i) From 14 to 17 hAPF, no major morphogenetic movement is observed $(A, B)$, yet epithelial cells of the dorsal thorax undergo a first round of division (fig. S10B and Movies S1, S2). The tissue undergoes only small local rotation (C). (ii) From 17 to $21 \mathrm{hAPF}$, a symmetric lateral to medial flow is observed within the lateral domain of the dorsal thorax ( $\left.A^{\prime}\right)$, corresponding to the contraction of the lateral domains of the dorsal thorax ( $\left.B^{\prime}\right)$ and its anterior-posterior elongation ( $B$ ' and Movie S3). This flow is associated with cell apical constriction in the lateral domain of the scutellum. Concomitantly, a medial to lateral flow is observed in the scutellum ( $A$ '), corresponding to the contraction-elongation of its medial domain ( $\left.B^{\prime}\right)$ and its dorsal-ventral elongation ( $B^{\prime}$ and Movie S3). During this period, extensive rotation is observed in the dorsal thorax $\left(C^{\prime}\right)$. (iii) From 21 to 36 hAPF, a symmetric 
lateral to medial flow and a major posterior to anterior flow are observed in the scutum (A"). These flow are associated with contraction-elongation deformation of its lateral domains (B") and its anterior-posterior elongation, as well as with the contraction of the pupa neck region. During this period the scutellum mostly expands in all directions (B"). High tissue rotation rates are observed in the lateral domain of dorsal thorax (C").

(A-C") Maps at 15 (A-C), 20 (A'-C') and 25 (A"-C") hAPF. (A-A") local velocities represented as vectors. (B-B") Deformation rates represented as ellipses: a direction of elongation is represented in red and contraction in blue. (C-C") Rotation rate represented by circle diameters: clockwise is represented in red and counter-clockwise. The scutellum is shaded in yellow and the scutum is outlined by a black dashed line. Scale bars: $100 \mu \mathrm{m}$ (black bars), $910^{-2} \mu \mathrm{m} \mathrm{min}^{-1}$ (arrows in A-A"), $2.410^{-3} \mathrm{~min}^{-1}$ (blue bars in B-B"), 8 $10^{-5} \min ^{-1}$ (circles in C-C").

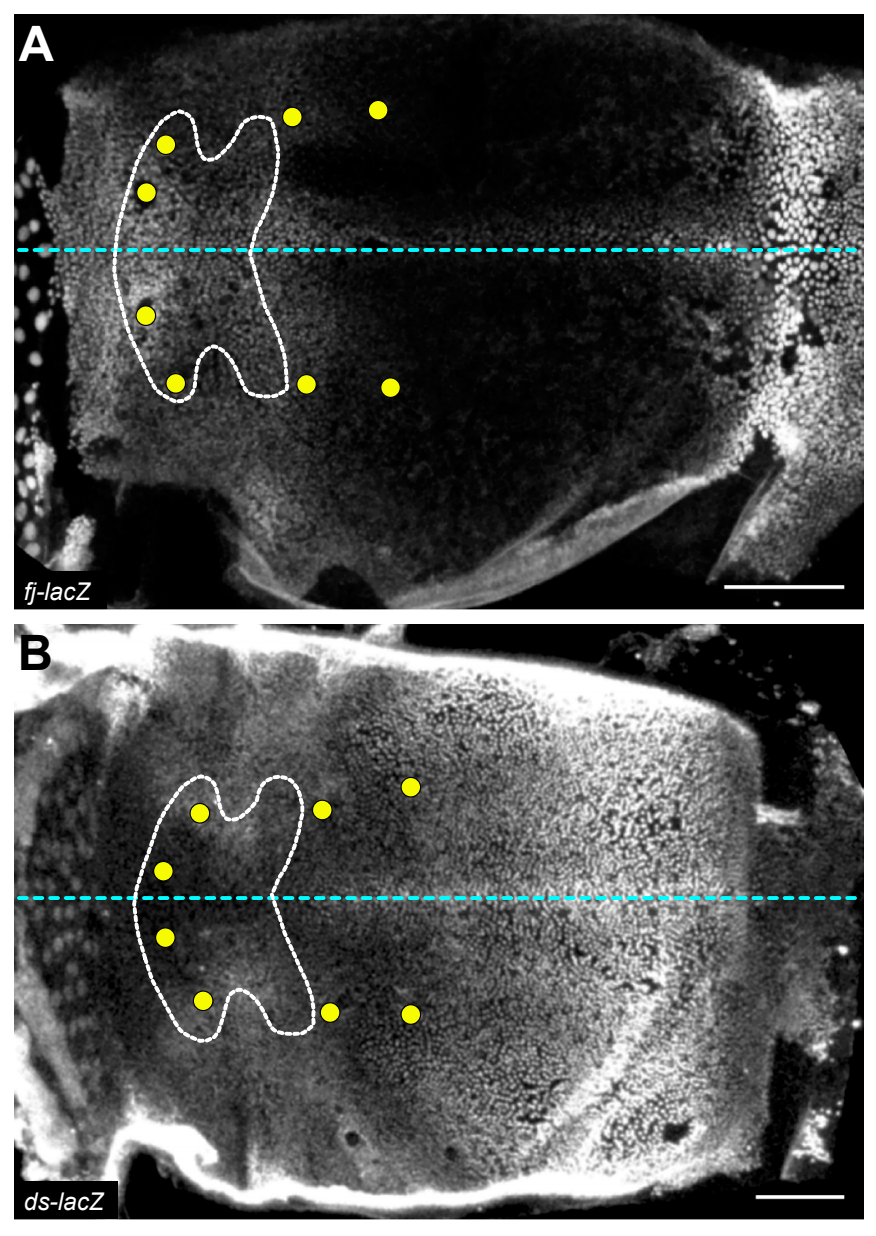

Figure S3. Gradients of $\boldsymbol{f} \boldsymbol{j}$ and $\boldsymbol{d} \boldsymbol{s}$ expressions are opposed in the scutellum. Whereas ds had an elaborate expression in the dorsal thorax, fj was mostly expressed in the medial domain of scutellum. Hence fj and ds are expressed in opposing domains in the scutellum and in the most posterior part of the scutum.

Anti- $\beta$ gal staining of (A) fj-lac $Z$ and (B) ds-lacZ in the pupa dorsal thorax at $19 \mathrm{hAPF}$. The scutellum is outlined by a white dashed line. Scale bars: $100 \mu \mathrm{m}$. 

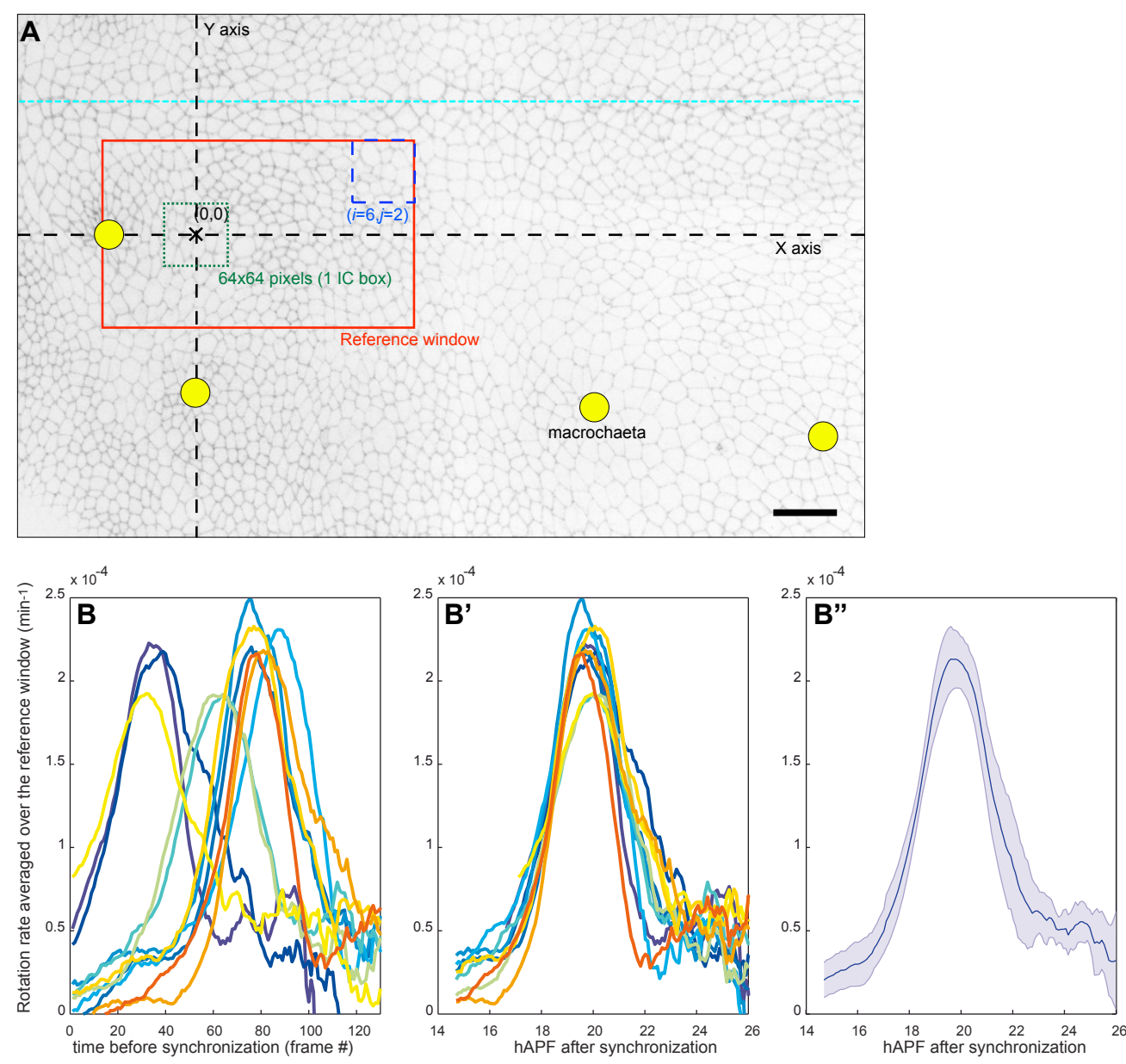

Figure S4. Spatio-temporal registration of movies. In order to compare different hemi-scutellum movies corresponding to the same genotype, and/or average their measurements to improve the signal to noise ratio, their common space and time coordinates were defined in two steps: first, spatial registration of the IC grid using the positions of the two scutellar macrochaetae (A); second, time registration using the point with the steepest slope before the rotation rate peak (B-B").

(A) Image of a wt hemi-scutellum at 16:30 hAPF showing the landmarks used for spatial adjustment between movies. The $X$ and $Y$ axes are determined using the positions of the macrochaetae at 16:30 hAPF. Red rectangle (IC-boxes labelled $i=-2$ to 6 and $j=-2$ to 2): window over which the rotation rate was measured and spatially integrated, called "reference window". Two IC-boxes are shown as dashed squares with their space coordinates $(i, j)$ indicated. (B-B") Synchronization: graphs showing the rotation rate of 11 wt movies at $29^{\circ} \mathrm{C}$ calculated in the reference window defined in A: (B) before and (B') after synchronization using as a reference 3/4 of the rotation peak in the ascent (set to 18:40 hAPF). (B") Graph showing the average rotation rate $\pm \mathrm{SD}$ of the same $11 \mathrm{wt}$ movies after space and time registrations. Scale bar: $20 \mu \mathrm{m}(\mathrm{A})$. 

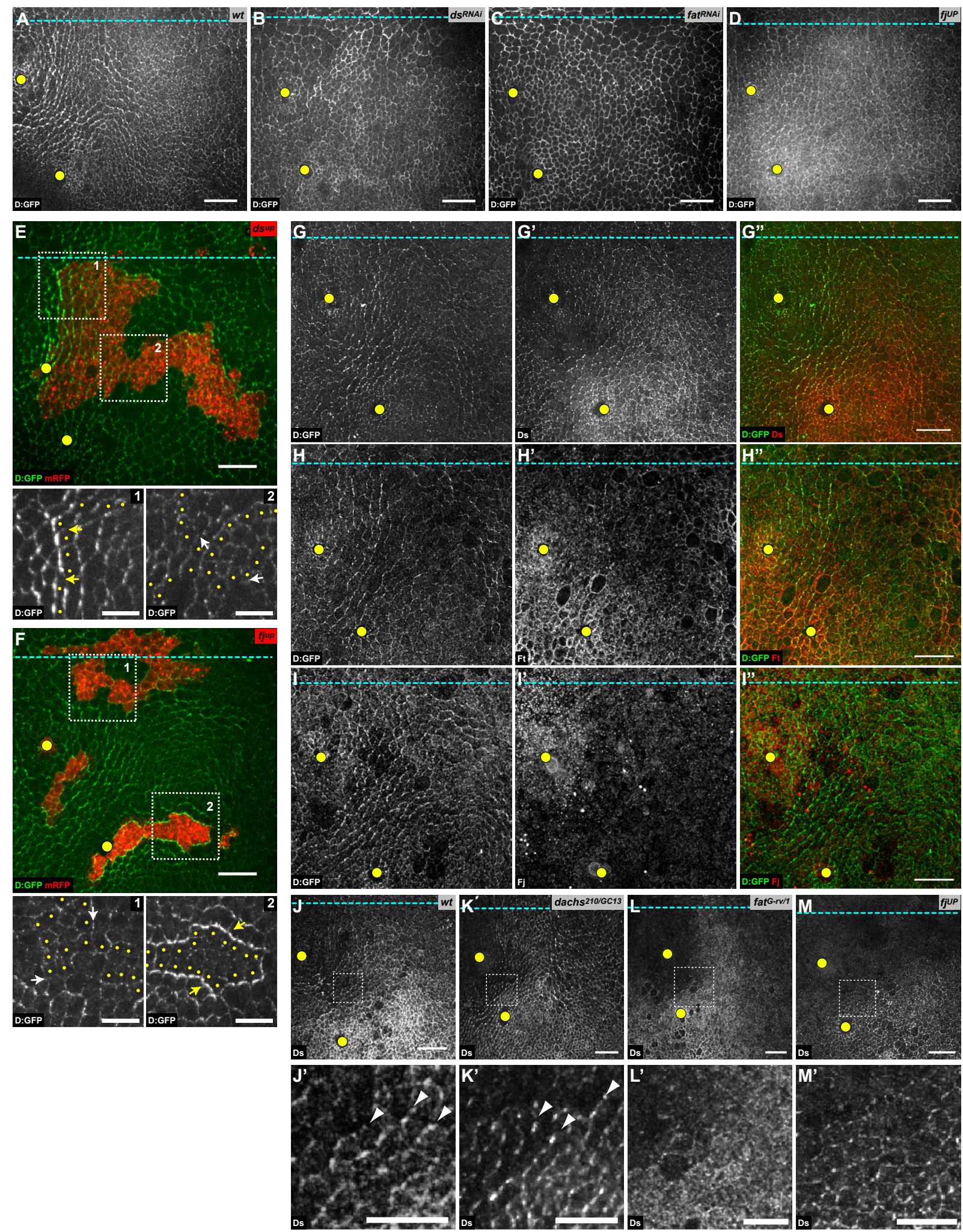

Figure S5. Ds planar polarization is independent of Dachs activity, but requires Fat activity as well as the graded distribution of $\mathbf{F j}$. Fat is enriched in some regions of the scutellum but is not as clearly polarized as Ds (compare G-G" and $H-H$ "). Fj is mostly localized in domains corresponding to the ones detected by fj-lacZ reporter (Fig. 2A'). Fj accumulates in small intracellular punctuated structures as 
expected for a Golgi resident protein. Ds is normally polarized in dachs $s^{210 / G C 13}$ tissue. In fat ${ }^{G-r v / 1}$ and $f j^{U P}$ pupae, Ds remains at the cell membrane, but Ds polarization is hardly detectable.

(A-D) Localization of D:GFP in wt (A), $d s^{R N A i}(\mathrm{~B}), f a t^{R N A i}(\mathrm{C})$ and $f j^{U P}(\mathrm{D})$ scutella. (E) Localization of D:GFP (green, gray in close-ups) in wt cells and in cells overexpressing $d s$ ( $d s^{U P}$ cells marked by mRFP expression, red). Close-ups in regions 1 and 2 show that $d s$ overexpression polarizes D:GFP in region 1 (yellow arrows), where $f j$ is highly expressed, but not in region 2 (white arrows) where $f j$ expression is low. Yellow dots indicate $d s^{U P}$ cells abutting the wt cells. (F) Localization of D:GFP (green, gray in close-ups) in wt cells and in cells overexpressing $f j$ ( $f j^{U P}$ cells marked by mRFP expression, red). Close-ups in regions 1 and 2 show that $f j$ overexpression polarizes D:GFP in region 2 (yellow arrows), where $d s$ is highly expressed, but not in region 1 (white arrows) where $d s$ expression is low. Yellow dots indicate $f j U P$ cells abutting the wt cells. (G-G") Localization of D:GFP (white in G, green in G") and Ds (white in G', red in G") in the scutellum. (H-H") Same for Fat. (I-I") Same for Fj. (J-M') Localization of Ds in wt (J,J'), dachs $s^{210 / G C 13}$ $\left(\mathrm{K}, \mathrm{K}^{\prime}\right)$ and $f a t^{G-r v / 1}$ (L,L') scutella, and in Fj overexpressing $f j{ }^{U P}$ (M,M') scutellum. J'-M' are close-ups of the regions outlined in J-M, respectively. Note that panels A to D and G to G" are extensions of the data presented in Fig. 2 of the main text. Scale bars: $20 \mu \mathrm{m}$ (A-M), $10 \mu \mathrm{m}$ (J'-M' and close-ups of E,F).

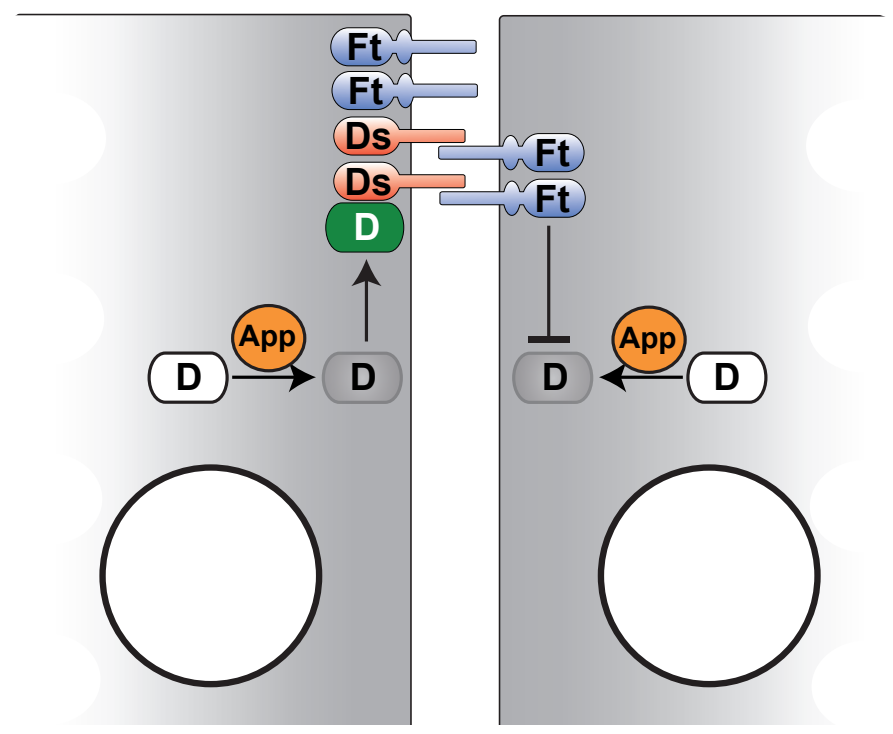

Figure S6. Regulation of Dachs asymmetric membrane localisation. Schematic: thin line, cell membrane; thick line, nucleus. Genetic evidences show that Fat activity inhibits Dachs membrane localisation $(6,49)$, whereas the DHHC Palmitoyltransferase Approximated (App) enzyme promotes Dachs membrane localisation (50). Fat and App are homogeneously distributed at the cell membrane (21, 50, 51). Our results showing that Ds is asymmetrically distributed and that Ds can interact with Dachs permit to propose that upon localisation of Dachs at the membrane by App, Ds polarizes Dachs distribution by two complementary mechanisms: (i) in an autonomous manner, Ds promotes Dachs asymmetric distribution by interacting with Dachs; (ii) in a non-autonomous manner, Ds polarizes Fat signalling in the neighboring cell, thereby excluding Dachs from the cell membrane facing the one where Ds is enriched. Fat processing and phosphorylation (not depicted) might contribute to the polarization of Fat activity in response to Ds binding $(52,53)$. 
A
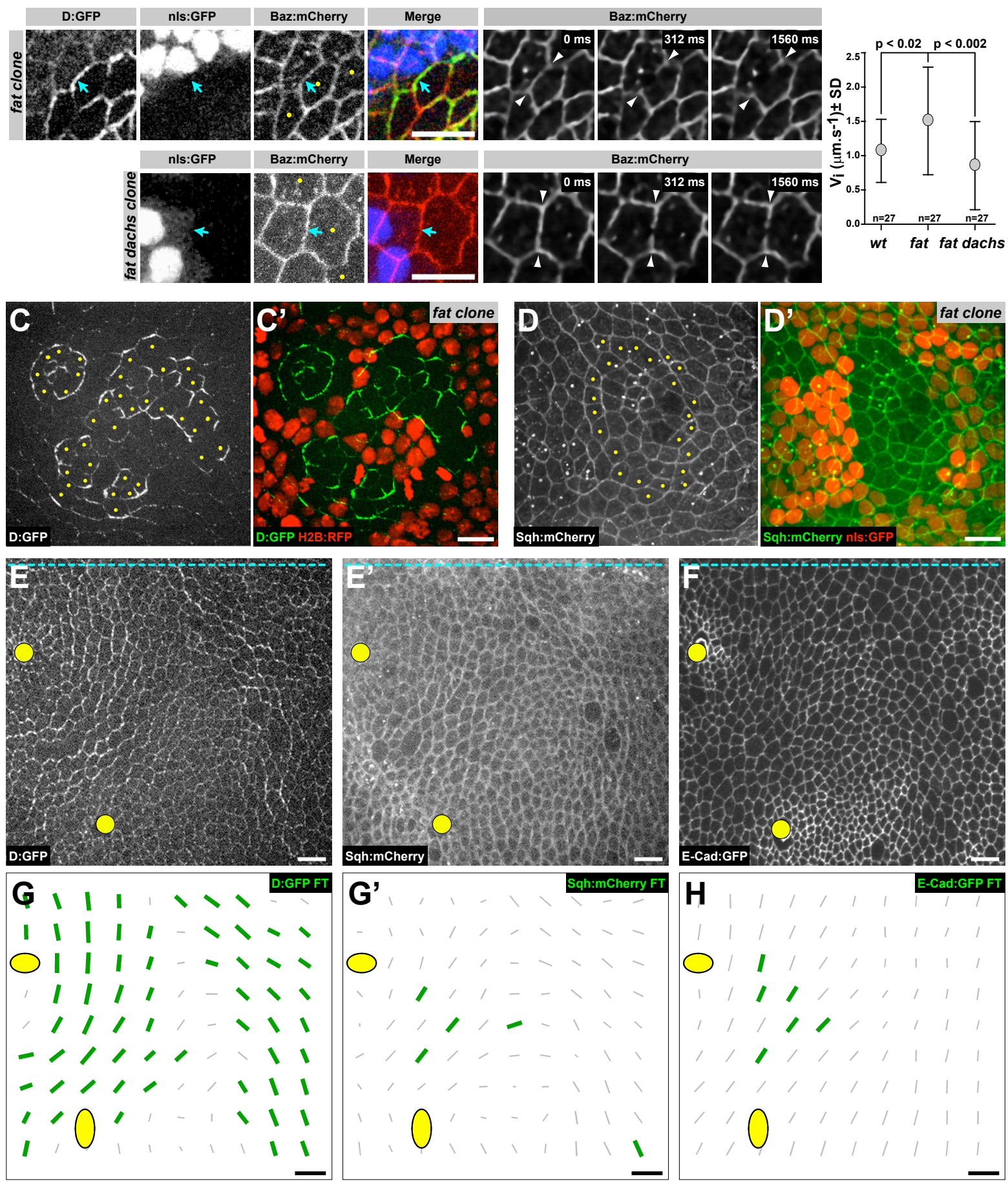

Figure S7. Regulation of tension anisotropy by Dachs polarization independently of MyosinII polarization. To assess whether Dachs regulates junction tension anisotropy independently of MyosinII polarization we performed several experiments. First we measured cell junction tension at the border between mutant and wt cells using laser ablation in mosaic tissues. It is established that fat clones trigger Dachs polarization at the interface between wt and fat mutant cells $(6,49)$. The interface between wt and mutant cells therefore provides an artificial boundary to analyse the role of Dachs polarization in junction tension by comparing the tension between wt and fat junctions and wt and fat, dachs double mutant junctions 
(A). Our ablation experiments revealed that indeed these ectopic cell junctions enriched in Dachs exhibited Dachs-dependent increase in cell junction tension (B). Furthermore, the interface between wt and fat cells does not show MyosinII polarization, as shown by Drosophila Myosin light chain (Spaghetti squash, Sqh) localization (compare $C$ and D). Finally, we have simultaneously recorded D:GFP and Sqh:mCherry during scutellum morphogenesis (E,E'). The quantification of their anisotropy by FT demonstrated that D:GFP is strongly and significantly polarized within the scutellum whereas Sqh:mCherry is not $\left(G, G^{\prime}\right)$. For comparison we also performed identical analyses on E-Cad:GFP movies during scutellum morphogenesis and found a nearly identical polarization pattern as we observed for the Sqh:mCherry (H). Both the Sqh:mCherry and E-Cad:GFP patterns were just below or just above the threshold at few places where cells are much elongated. Everywhere else, they are not significantly different from zero. Combined, these results demonstrate that Dachs polarization results in anisotropic interfacial tension. The molecular mechanism of how Dachs generates tension remains to be characterized. However, Dachs is most closely related to the well characterized MyosinV and MyosinI (54) which have been shown to produce movement on actin using in vitro assays (55, 56). Furthermore, sequence analyses of Dachs demonstrated that all the necessary domains to be an actin motor (actin bindings site, nucleotide binding domain) are present and hence to produces force (A. Houdusse personal communication). Dachs mostly differs from MyosinV or I in the transducer domain, which determines the processivity of the myosin motor. Based on the sequence comparison of Dachs with MyosinV or MyosinI, which have been crystallized and biochemically characterized (57, 58), the most likely scenario is that the interfacial tension is generated by the contractile force generated by the motor activity of the MyosinDachs in complex with proto-cadherin Dachsous.

(A) Representative images of cell junction ablation between wt and fat cells (upper panels) and between wt and fat dachs cells (lower panels) prior to ablation and after ablation (top right corner of each panel indicates the time relative to ablation). Mutant cells are marked by the absence of nls:GFP. Yellow dots indicate mutant cells abutting wt cells. Arrows indicate the ablated junctions and arrowheads the position of the vertices. Cell junctions between wt and fat cells have increased amounts of D:GFP (first panel and see also C). Ablations at junctions between wt and fat cells and control ablations (between differently marked wt cells) were performed in pupae expressing both D:GFP and Baz:mCherry, while ablations of junctions between wt and fat dachs cells were performed in pupae that expressed only Baz:mCherry. All ablations have been performed in the scutum to avoid the endogenous polarization of Dachs. (B) Graph of the mean initial relaxation speeds of vertices in junctions between wt and wt cells $\left(1.07 \pm 0.46 \mu \mathrm{m} \mathrm{s}^{-1}, n=27\right)$, wt and fat mutant cells $\left(1.50 \pm 0.78 \mu \mathrm{m} \mathrm{s}^{-1}, n=27\right)$ and wt and fat dachs double mutant cells $(0.85 \pm 0.64$ $\mu \mathrm{m} \mathrm{s}^{-1}, n=27$ ). (C,C') D:GFP (grey in C and green in C') in wt and fat cells. Mutant cells are marked by the absence of H2B:RFP (red in C'). D:GFP is enriched at the interface between wt and fat cells. (D,D') Sqh:mCherry (grey in D and green in D') in wt and fat cells. Mutant cells are marked by the absence of nls:GFP (red in D'). No polarization of Sqh:mCherry can be detected at the interface between wt and fat cells. (E,E') Images of the scutellum at 19 hAPF of a pupa expressing D:GFP (E) and Sqh:mCherry (E'). (F) E-Cad:GFP in the scutellum at 19 hAPF. (G,G',H) FT analyses of E,E',F averaged between 17:20 and 21:20 hAPF ( $n=3$ for each panel): maps of the D:GFP $(G)$, Sqh:mCherry (G') and E-Cad:GFP (H) anisotropy. Significant data: green bars; other: gray bars. In all panels, yellow dots indicate mutant cells abutting wt cells. Scale bars: $10 \mu \mathrm{m}$. 

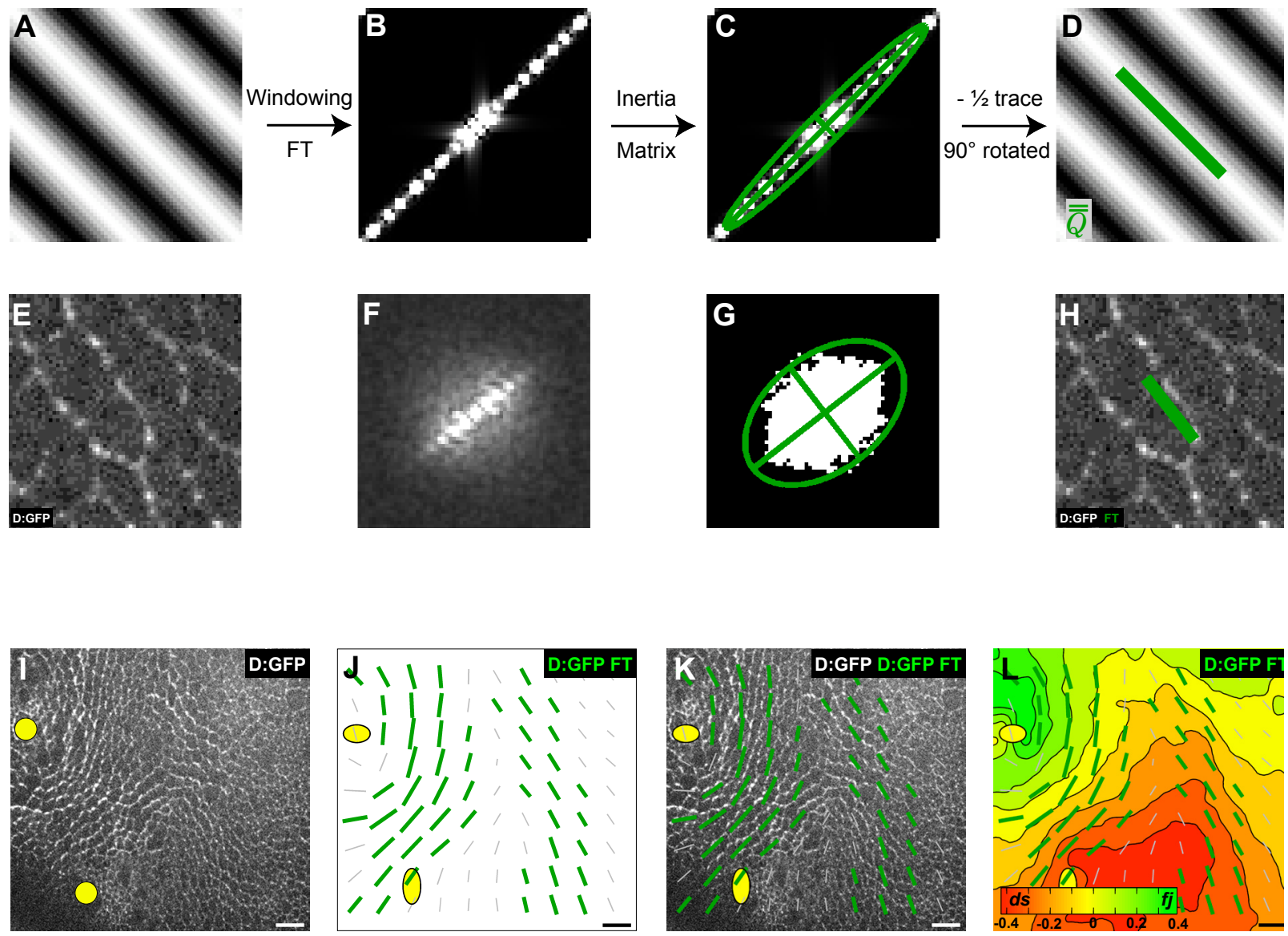

Figure S8. Quantification of D:GFP pattern anisotropy. A green bar is plotted in the direction of D:GFP pattern anisotropy, quantified by $2 D$ discrete Fourier transform (FT) analysis used to build the $\overline{\overline{\mathbf{Q}}}$ matrix (section 2.3.3. It correlates with the position and shape of the opposing fj and ds gradients pattern.

(A-D) FT and construction of $\overline{\overline{\mathbf{Q}}}$ on a basic example. (A) Periodic sinusoidal pattern tilted at $45^{\circ}$.

FT norm of image A represented in the Fourier space, after an appropriate windowing (multiplication by a square cosine) that minimizes the edge effects. The origin of the Fourier space has been shifted so that it is at the center of the panel in the Fourier space, rather than in a corner. (C) Inertia matrix of the pixels in B that are above the $80^{\text {th }}$ percentile, represented as a green ellipse and overlayed to B. (D) The matrix obtained in $\mathrm{C}$ has its trace removed, the square root of its positive eigenvalue is represented after $90^{\circ}$ rotation and multiplication by the contrast of A (here equal to 1). The resulting green bar represents $\overline{\overline{\mathbf{Q}}}$ which quantifies the anisotropy and orientation of the pattern visible in A. (E-H) FT and construction of $\overline{\overline{\mathbf{Q}}}$ for an actual Dachs signal. (E) Raw image extracted from a time-lapse D:GFP movie, cropped in an IC-box.(F) Same as B, averaged over 2 h. (G) Same as C. (H) Same as D. (I-L) Pattern of D:GFP polarization. (I) Snapshot of D:GFP pattern. An additional polarization domain is present at the scutellum-scutum boundary, again at a position where the opposing $f j$ and $d s$ gradients meet. $(\mathrm{J})$ Map of the D:GFP magnitude and anisotropy determined by Fourier Transform (FT) on the average of D:GFP time-lapse movies $(n=3)$ between 17:20 and 21:20 hAPF. Significant data: thick green bars; other: thin gray bars. (K) Overlay of (I) and (J). (L) Overlay of (J) and fig. 2A". Scale bars: $10 \mu \mathrm{m}$ (I-L). Box size: $64 \times 64$ pixels $\sim 20 \times 20 \mu \mathrm{m}^{2}$ (A,D,E,H). 


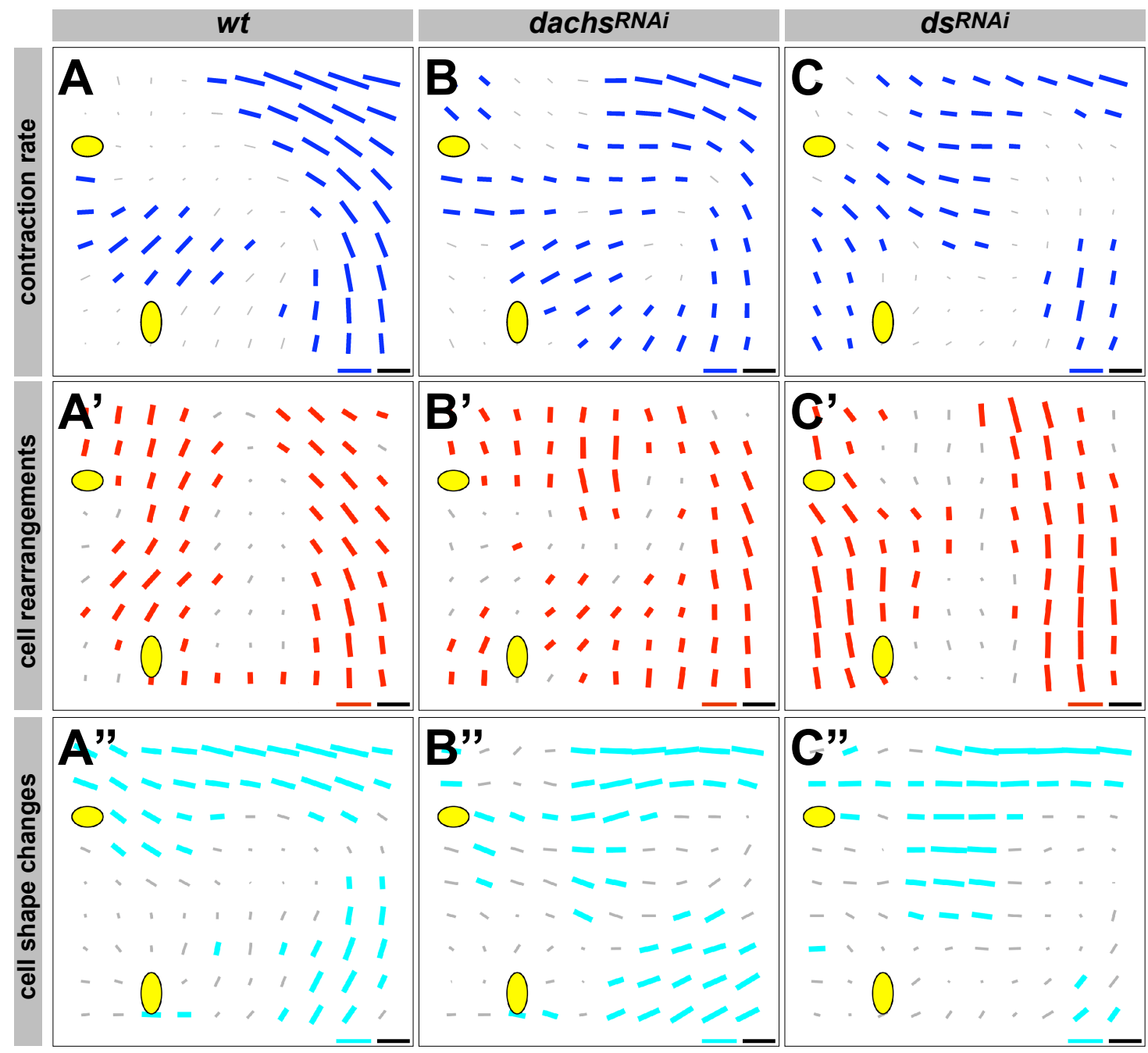

Figure S9. Contraction rates, cell rearrangements and cell shape changes in wt, dachs ${ }^{R N A i}$ and $\boldsymbol{d} \boldsymbol{s}^{R N A i}$ hemi-scutella. Having observed similarities between the wt contraction rate map (A) and the Dachs signal pattern (Fig. 4A), we looked for the specific contribution to flow of Dachs and Ds (other mutations of the Fat/Ds/Fj pathway are presented in fig. S14). The flow in wt, dachs ${ }^{R N A i}$ and $d s^{R N A i}$ tissues was calculated according to the right hand side of Eq. (10b). Using the flow presented here (B-C), we subtracted the contraction rates of dachs ${ }^{R N A i}$ from $w t(A-B)$, and of $d s^{R N A i}$ from $w t(A-C)$, according to Eq. (10b). This yielded the maps presented in Fig. 4C,D. We then used the method explained in section 4.2 to measure the contributions of cell rearrangements ( $A$ '- $C$ '), and of cell shape changes ( $A$ "- $C$ ") to tissue contraction. They too are represented as bars, as explained in fig. S11.

(A-C") Maps of local contraction rate $\overline{\overline{\nabla \mathbf{V}}}^{\text {dev }}$ (A-C), traceless parts of rearrangement matrix (A'-C') and of cell shape change matrix (A"-C") between 17:20 and 21:20 hAPF in wt(A-A"), dachs ${ }^{R N A i}(\mathrm{~B}-\mathrm{B}$ "), and $d s^{R N A i}(\mathrm{C}-\mathrm{C} ")$ scutella. Significant values are shown as thick colored bars, others are shown as thin gray bars. Yellow ellipses indicate the ensemble average \pm SD positions of the macrochaetae. Scale bars: $10 \mu \mathrm{m}$ (black bars); $5.510^{-4} \mathrm{~min}^{-1}$ (blue bars in A-C); $1 \mu \mathrm{m}^{2} \mathrm{~min}^{-1}$ (red bars in A'-C', cyan bars in A"-C"). 


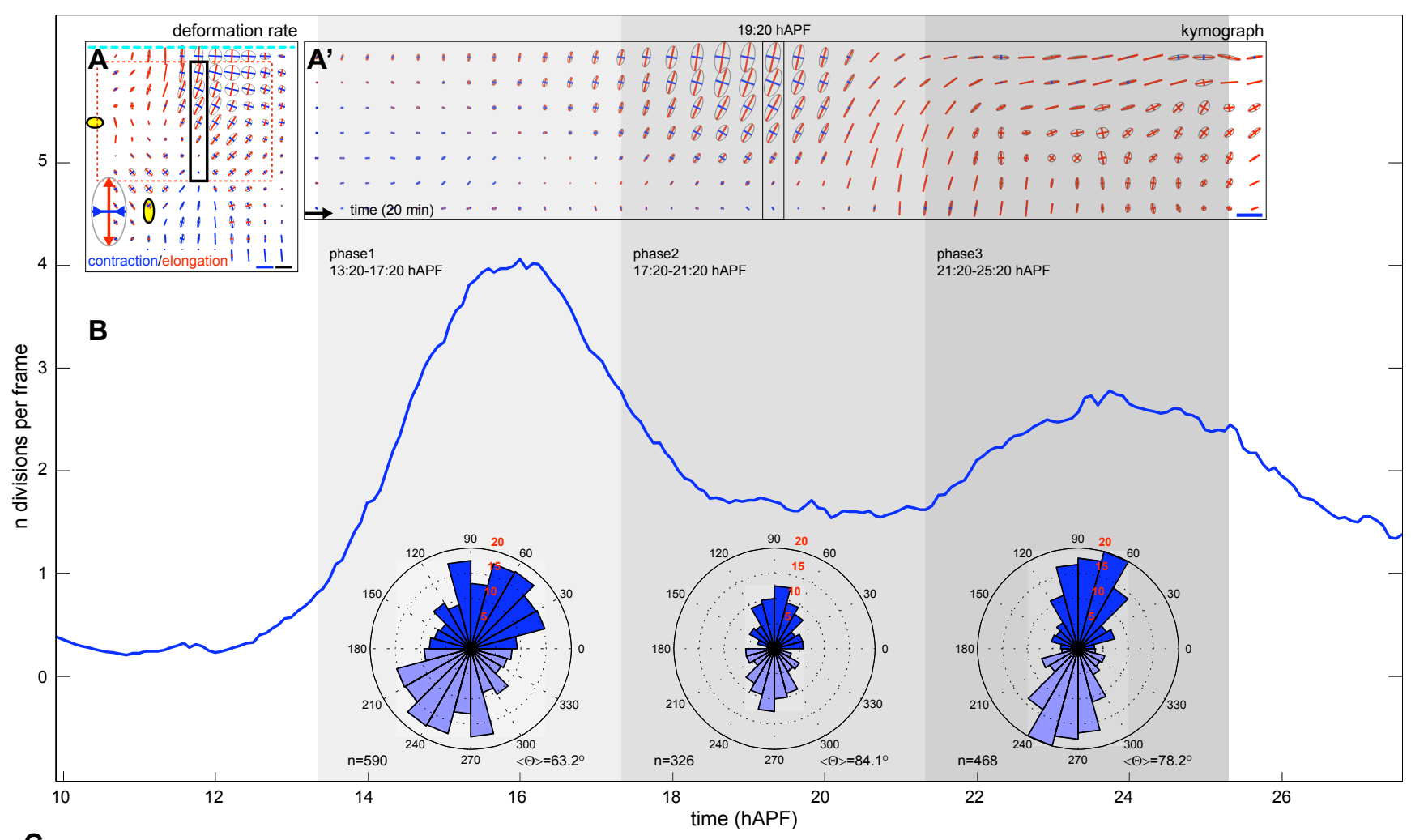

C
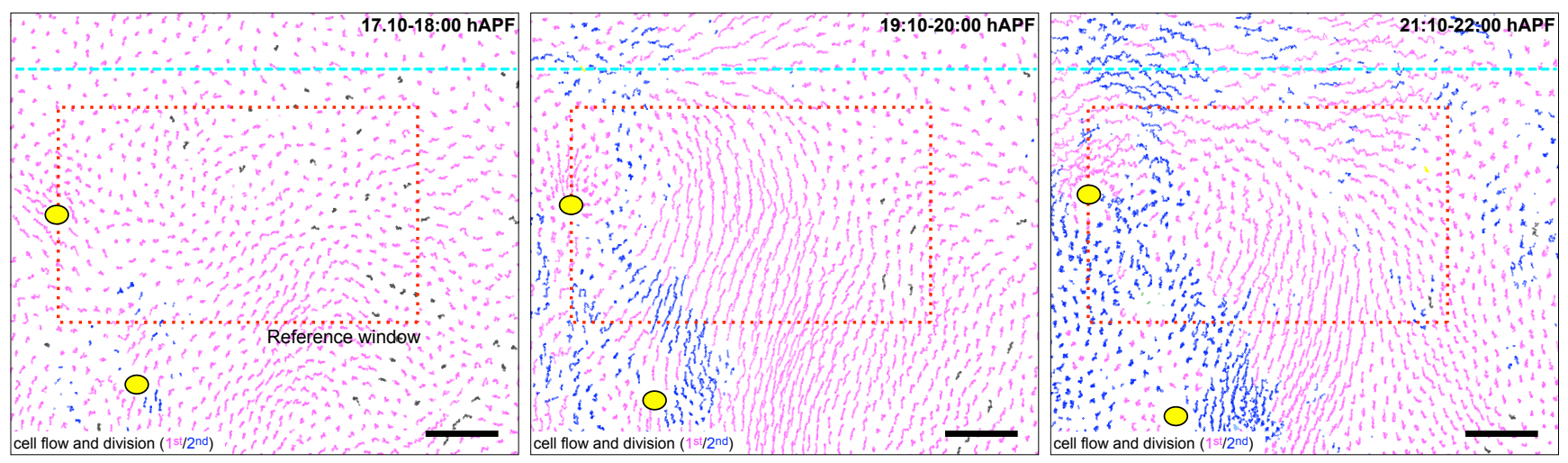

Figure S10. Contraction-elongation of the scutellum medial region does not correlate with cell divisions. To investigate the role of cell division orientation in scutellum morphogenesis, we have first compared the magnitude of tissue deformation with the timing and the orientation of cell division (A, $A$ ' and $B)$. Second, we have compared the trajectories of cells with their division timing $(C)$. We found three phases: phase 1, before 17:20 hAPF no deformation is observed; phase 2, between 17:20 and 21:20 hAPF the medial domain undergoes a contraction-elongation movement, and most cells of the scutellum medial domain move laterally without undergoing cell division; phase 3, after 21:20 hAPF the tissue expands in all directions (A'). During these three phases cell divisions are aligned along the tissue medial-lateral axis. The comparison of the cell division rate with the magnitude of tissue deformation reveals that: (i) cell division rate, maximal in phase 1, does not correlate with the magnitude of tissue deformation, minimal in phase 1; (ii) the strongest contraction-elongation deformation of the scutellum medial domain (phase 2) occurs between two waves of 
cell divisions (phases 1 and 3); (iii) during each wave of cell divisions (phases 1 and 3), the orientation of cell division does not correlate with that of tissue deformation rate. Together, these results argue against a role of cell division orientation in the contraction-elongation deformation of the scutellum medial region

(A,A') Local deformation rate averaged over $5 \mathrm{wt}$ hemi-scutellum movies at $25^{\circ} \mathrm{C}$ between $13: 20$ and 26:40 hAPF, represented as ellipses: a direction of elongation is represented in red and contraction in blue. (A) Local deformation rate averaged between 17:20 and 21:20 hAPF. The solid and dashed rectangular boxes outline the domains where the kymograph in $\mathrm{A}^{\prime}$ and the cell division orientation in $\mathrm{B}$ are measured, respectively. (A') Kymograph of the deformation rate in the medial domain of the scutellum (solid box in A). Note that the data presented in panels A-A' are extensions of the data presented in Fig. 2B. (B) Division rate: graph of the ensemble average number of cell divisions per frame and symmetrized rose plots of their orientation during the three phases of development measured in the dashed box in A, obtained by segmenting and tracking each individual cell between 13:20 and 26:40 hAPF. The number of cell divisions per frame was time averaged over 24 frames and ensemble averaged over 4 wt hemi-scutellum movies at $25^{\circ} \mathrm{C}$ (total cumulated over 4 movies: $n=1591$ divisions, with an error rate estimated $<1 \%$ by visual inspection of each frame). (C) 50 min cell trajectories during contraction-elongation movements. Following the segmentation of a single time-lapse movie of the scutellum between 11:00 and 27:40 hAPF, cell lineages, timing of each division and cell trajectories were determined. Cell trajectories are color coded according to the number of cell divisions that each cell has undergone: pink for cells having divided once; blue for cells having divided twice; green (a few cells are visible between the macrochaetae) for the third wave of division which begins just before $22 \mathrm{hAPF}$ (Movie S2B). The rectangular box (reference window, same as in fig. S4A) outlines the domain where cell division orientations are measured in (B). Scale bars: $10 \mu \mathrm{m}(\mathrm{A}) ; 10^{-3} \mathrm{~min}^{-1}$ (blue bars in $\mathrm{A}$ and $\mathrm{A}^{\prime}$ ); $20 \mu \mathrm{m}(\mathrm{C})$. 


\section{A}
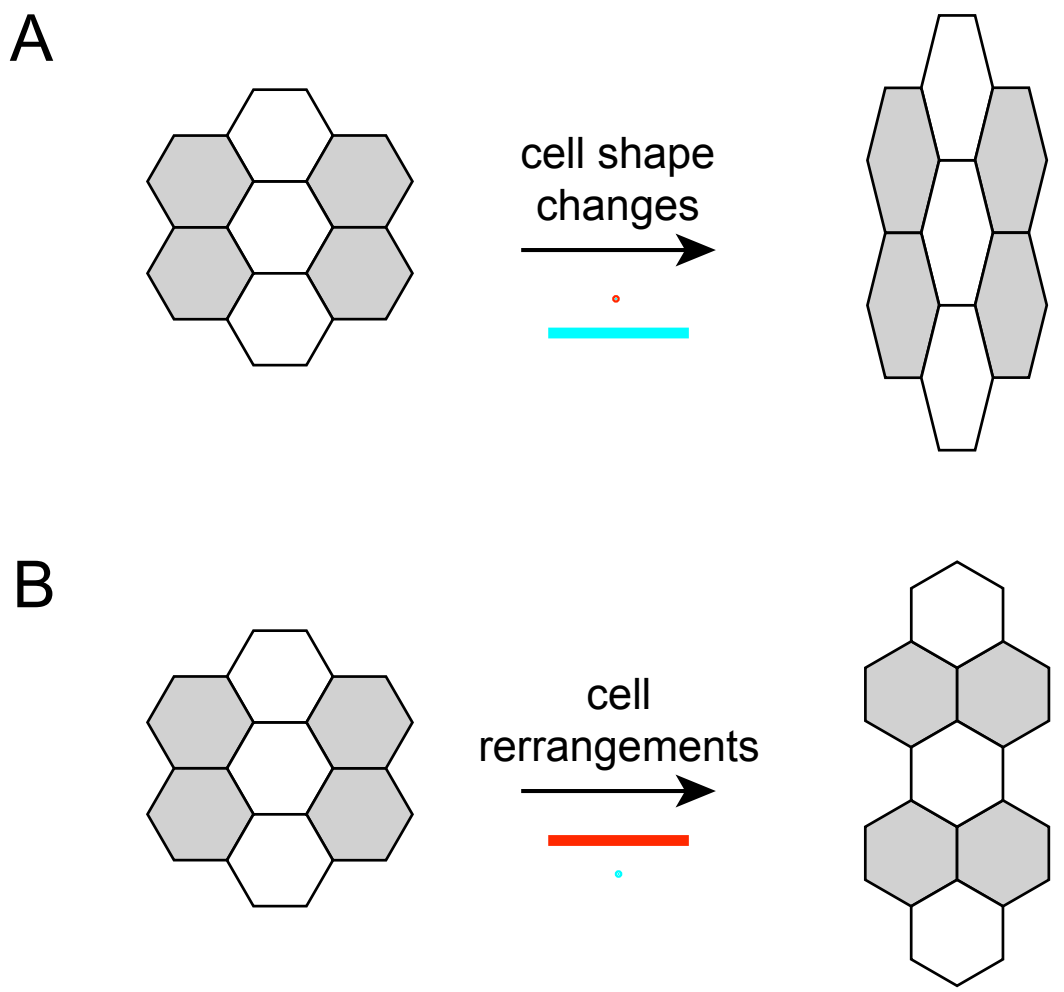

Figure S11. Representation of quantitative analysis of cell rearrangements and cell shape changes. Between the initial (left) and final (right) images, a tissue domain is undergoing a contraction (here along the horizontal axis) accompanied by an elongation in the perpendicular direction. Such tissue domain shape change may arise from processes such as cell rearrangements and changes of individual cell shapes. For simplicity, cells are here sketched as identical, and both processes are depicted separately, but the same quantitative analysis was performed on our experimental movies of the developing Drosophila dorsal thorax where both processes may contribute to local tissue deformations.

(A) Cell shape changes, represented by a cyan bar quantifying the number, the magnitude and the direction of cell contraction (here horizontal). Since in this example all cells conserve their neighbors, cell rearrangements have no contribution (red dot). (B) Round of oriented cell rearrangements: horizontal junctions shrink and disappear, new vertical junctions appear and elongate. Cell rearrangements are represented by a red bar quantifying the number, the length and direction of lost junctions (here horizontal). Since in this example all cells end up with the same shape as in their initial state, cell shape changes have no contribution (cyan dot). 


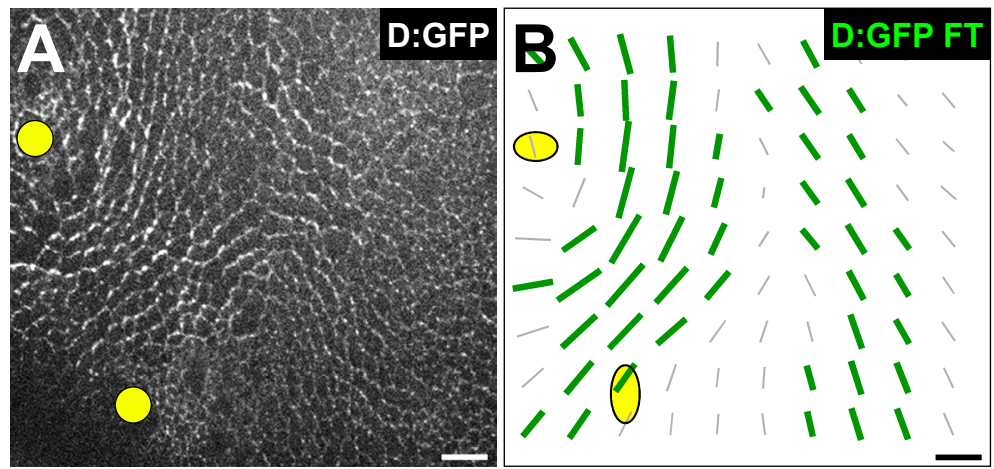

\section{alignment coefficient}

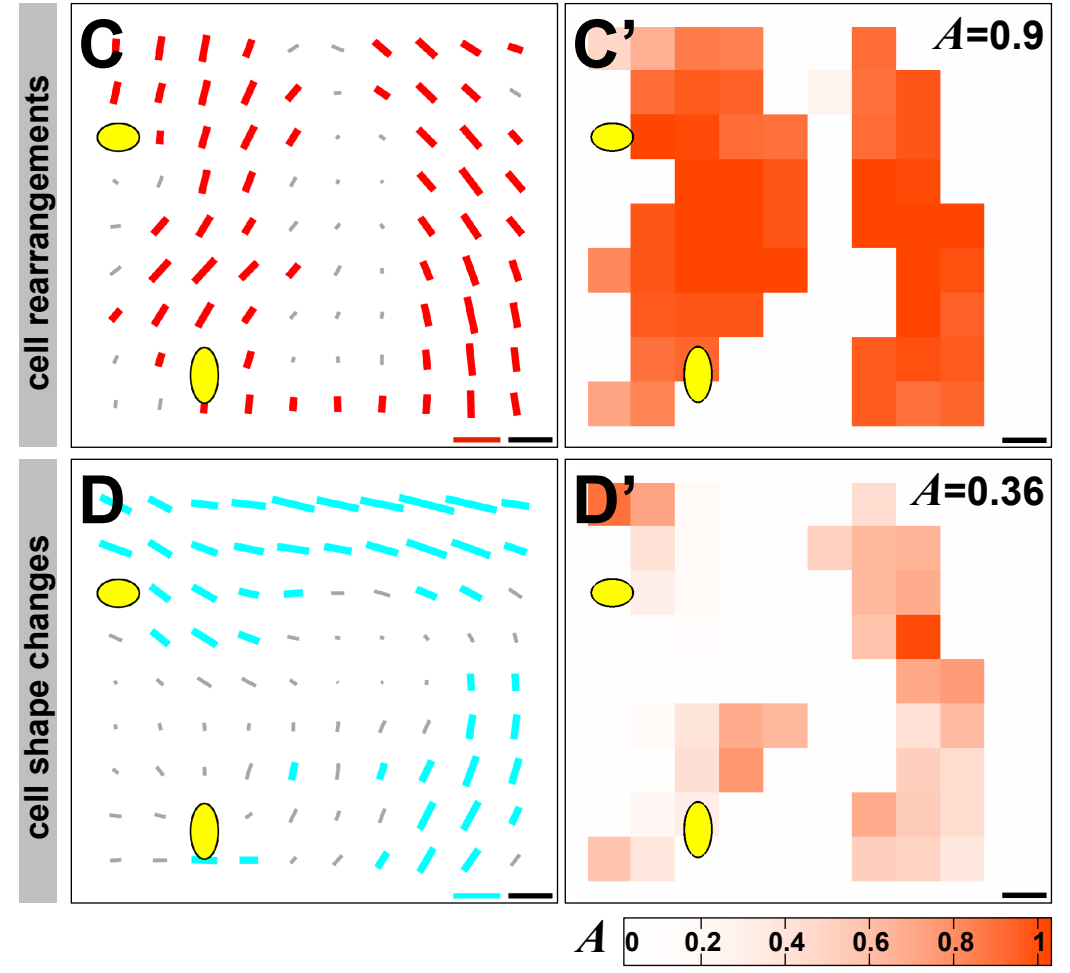

Figure S12. Dachs polarization, as quantified by Fourier Transform (FT), strongly correlates with cell rearrangements and poorly correlates with cell shape changes.

(A,B) D:GFP pattern: (A) snapshot, the same as Fig. 2C, Fig. 4A, fig. S5A and fig. S8I. (B) Averaged map of FT, the same as Fig. 4B and fig. S8J. (C,C') Maps of cell rearrangements in wt (C) and of their alignment coefficients with the D:GFP FT (C'). (D,D') Same for cell shape changes. Significant data: color bars; other: gray bars $(\mathrm{C}, \mathrm{D})$. The local score of the alignment with D:GFP FT (C',D') is coded in orange from 0 for fully anti-correlated, to 1 for fully correlated, through 0.5 for non correlated. The overall score A is indicated. Scale bars: $10 \mu \mathrm{m}$ (A-D'), $1 \mu \mathrm{m}^{2} \mathrm{~min}^{-1}$ (red bar in C, cyan bar in D). 


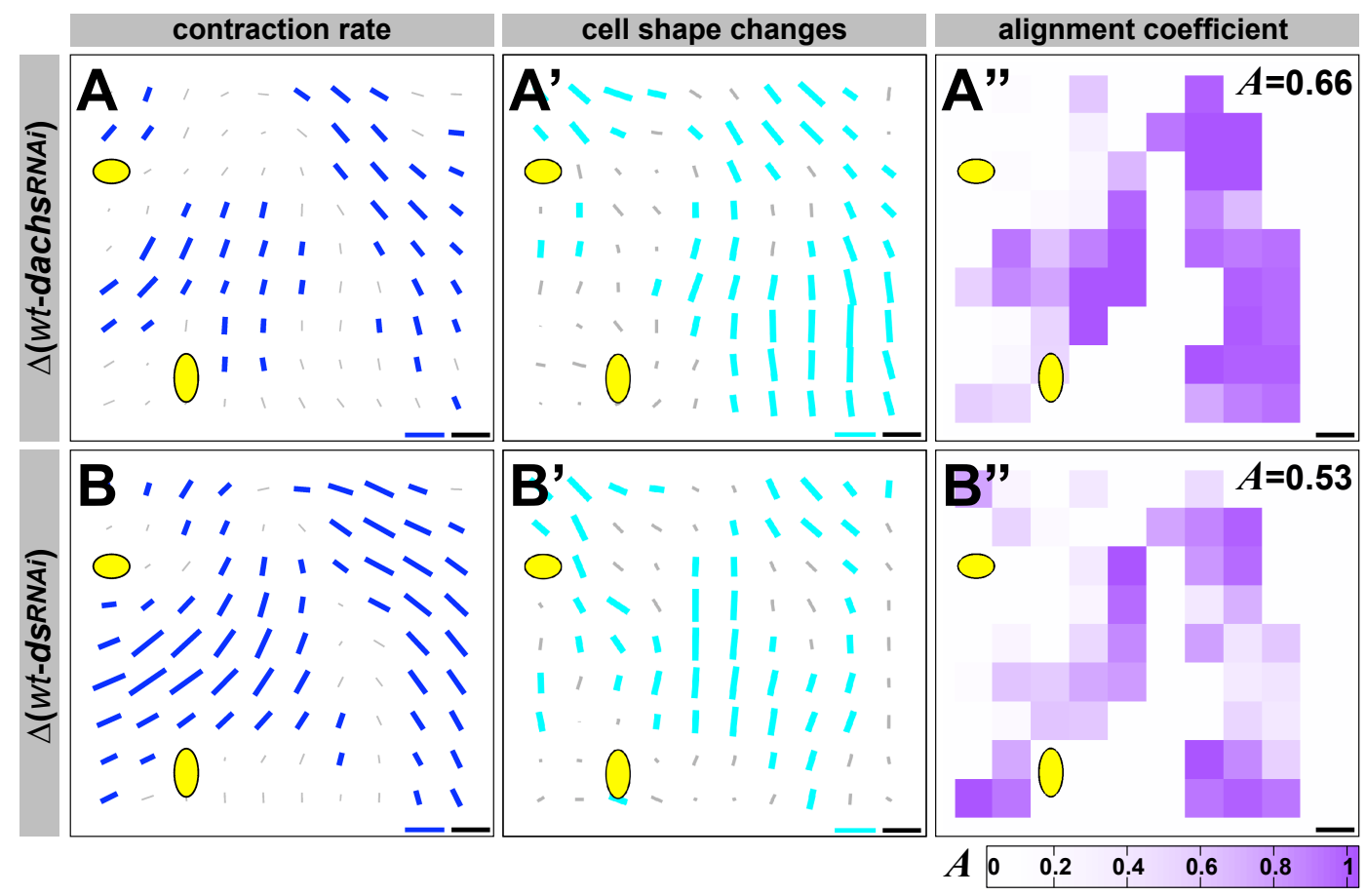

Figure S13. Maps of local cell shape changes and of their correlation with contraction rates in dachs $^{R N A i}$ or $\boldsymbol{d s}^{R N A i}$ mutant conditions.

(A-B) Specific contribution of $d a c h s^{R N A i}(\mathrm{~A})$ and $d s^{R N A i}(\mathrm{~B})$ to contraction rate, calculated according to the right hand side of Eq. (10b). Same panels as in Fig. 4C,D. (A'-B') Specific contribution of $d a c h s^{R N A i}$ $\left(\mathrm{A}^{\prime}\right)$ and $d s^{R N A i}\left(\mathrm{~B}^{\prime}\right)$ to cell shape changes: the cell shape change (explained in section 4.2) of $d a c h s^{R N A i}$ or $d s^{R N A i}$ mutant condition is subtracted from that of wt one. (A"-B") Maps of the alignment coefficient between the specific contraction rate (A-B) and cell shape changes (A'-B') in $d a c h s^{R N A i}\left(\mathrm{~A}^{\prime \prime}\right)$ and $d s^{R N A i}$ (B"). Data are represented only where D:GFP has a significantly non-zero FT signal. The local score of the mutual alignment is coded in purple from 0 for fully anti-correlated, to 1 for fully correlated, through 0.5 for non correlated. The overall score A is indicated: cell shape changes score 0.66 and 0.53 , while cell rearrangements score a significantly higher correlation at 0.8 and 0.77 , respectively (Fig. 4E',F'). Scale bars: $10 \mu \mathrm{m}, 5.510^{-4} \mathrm{~min}^{-1}$ (blue bars in A, B), $1 \mu \mathrm{m}^{2} \mathrm{~min}^{-1}$ (cyan bars in A', B'). 

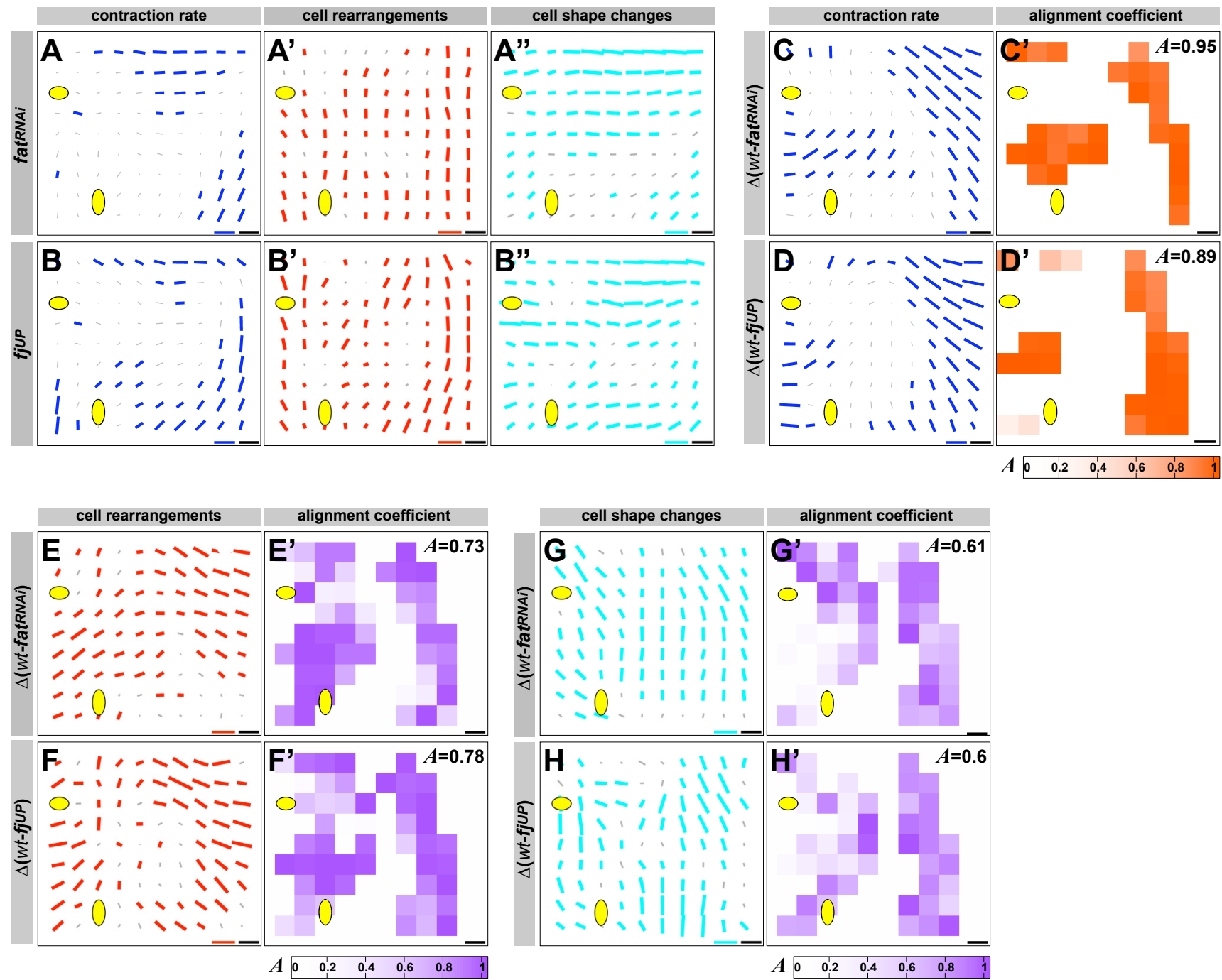

Figure S14. Analysis of tissue contractions, cell rearrangements and cell shape changes in $f j^{U P}$ and fat $^{R N A i}$ scutella. To facilitate the comparison with Dachs and Ds analyses, the legend is the same as in the corresponding figures: (A-B") fig. S9B-C", (C-F') Fig. 4C-F', (G-H') fig. S13A"-B". Note the similarity of Fat data with Dachs and Ds. This analysis of cell rearrangements and cell shape changes in $f j^{U P}$ and fat ${ }^{R N A i}$ scutella confirmed the results obtained for dachs ${ }^{R N A i}$ and $d s^{R N A i}$, namely that Dachs controls tissue contraction mainly by promoting cell rearrangements along its polarization pattern.

(A-H') Maps for $f j{ }^{U P}$ and $f a t^{R N A i}$. Color-coded maps represent alignment coefficients between the difference in contraction rate and: the D:GFP FT (C',D': orange); cell rearrangements within the D:GFP FT pattern (E',F': purple); and cell shape changes within the D:GFP FT pattern (G',H': purple). Overexpression of $f j$ partially abrogated D:GFP polarity mostly in the anterior part of the $\Lambda$-shaped domain and, accordingly, significant differences in the convergence rate were observed mostly in the anterior part (D). 

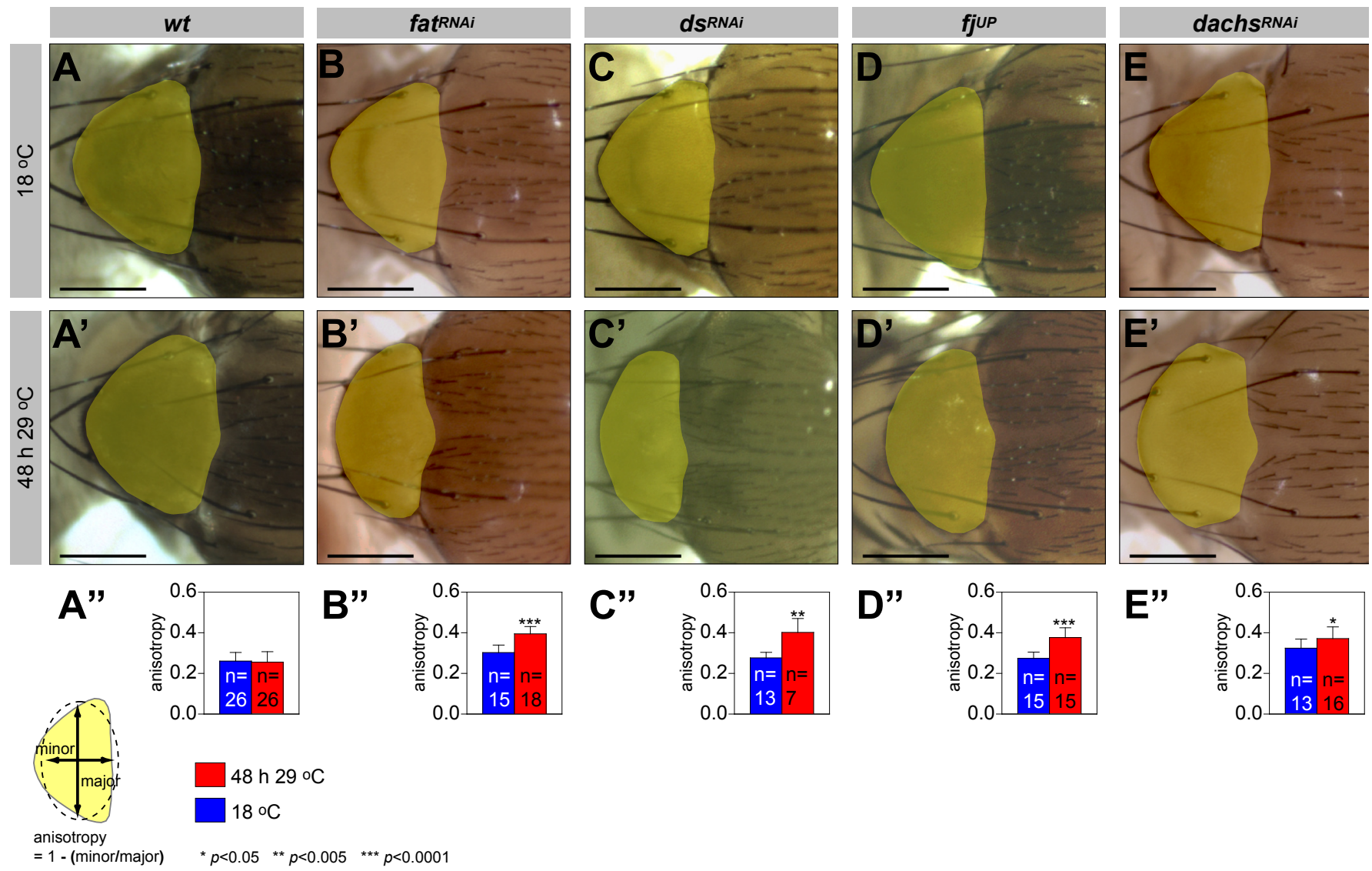

C"

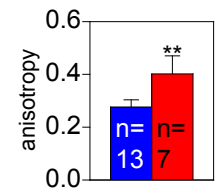

D"
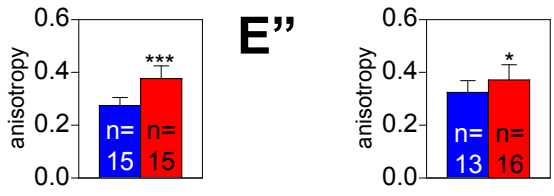

Figure S15. The Fat/Ds/Fj/Dachs pathway modifies the scutellum shape. $f a t^{R N A i}$ ( $B-B$ "), $d s^{R N A i}(C-C "), f j{ }^{U P}(D-D ")$, and dachs ${ }^{R N A i}$ (E-E") mutants in which we temporally abrogated the gradient of fj expression or abrogated the function of $d s$, fat or dachs using the GAL80ts (13) resulted in the elongation of the adult scutellum along its medial-lateral axis and in a higher anisotropy (defined in inset). These mediallateral elongations agree with the respective differences in contraction rates: the medio-lateral contraction in wt is replaced in mutants with an antero-posterior contraction (Fig. 4 and fig. S9).

(A-E") Adult scutella (shaded yellow domains in A-E') and histograms (A"-E") of their anisotropy (defined in inset) for wt (A-A"), fat ${ }^{R N A i}$ (B-B"), $d s^{R N A i}$ (C-C"), $f j^{U P}$ (D-D"), and dachs ${ }^{R N A i}$ (E-E") adults that were raised at $18^{\circ} \mathrm{C}$, used as controls (A-E), or that were transferred to $29^{\circ} \mathrm{C}$ for a period of $48 \mathrm{~h}$ ( $\left.\mathrm{A}^{\prime}-\mathrm{E}^{\prime}\right)$. Change in temperature from $18^{\circ} \mathrm{C}$ to $29^{\circ} \mathrm{C}$ did not affect the wt scutellum shape. $p$ values were determined by Student's $t$-test. Scale bars: $250 \mu \mathrm{m}$. 


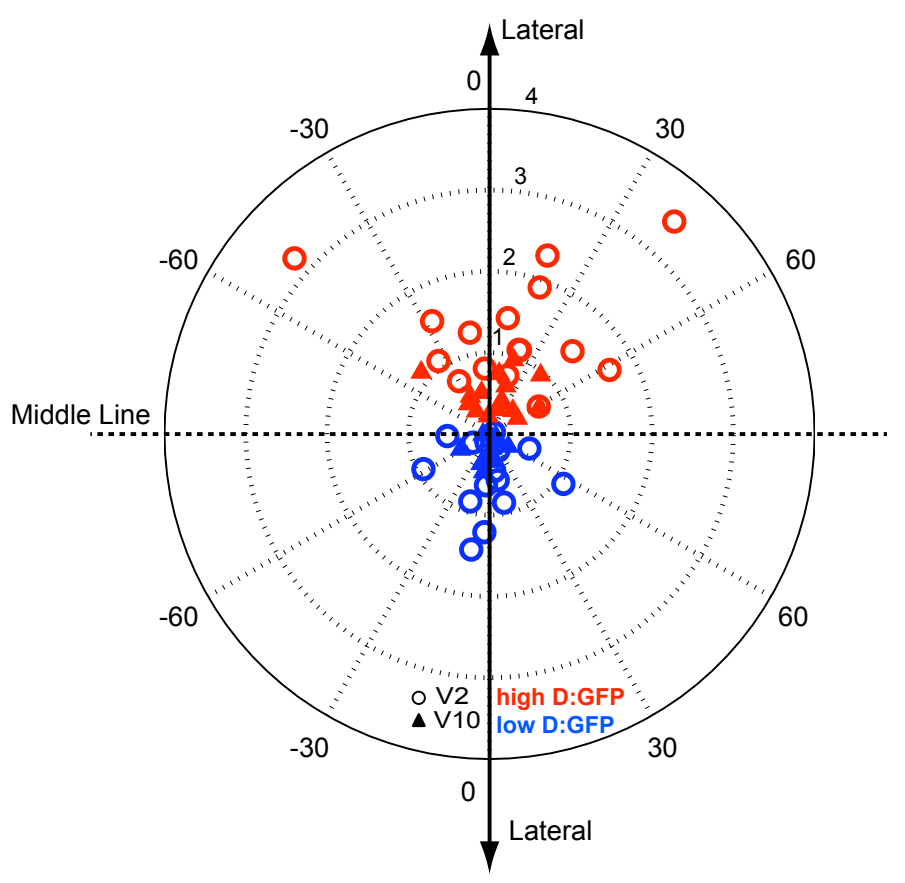

Figure S16. Orientation and tension of a cell junction do not correlate. The D:GFP enriched junctions can be found at various orientations relative to the anterior-posterior animal axis (Fig. 4A) and we have ablated an unbiased sample. We find that the amplitude of relaxation speed, and thus the amplitude of the tension, does not correlate with the direction of the junction prior to ablation. We interpret this absence of correlation by stating that the junction tension depends on its level of Dachs, while it does not depend on its orientation.

Plot of the initial relaxation speed $\left(\mu \mathrm{m} \mathrm{s}^{-} 1\right)$ of vertices after ablation of cell junction with high (red) or low (blue) D:GFP levels, versus direction of the junction prior to ablation, plotted in two symmetrical hemicircles for clarity. Circles are $V_{2}$ and triangles are $V_{10}$ (see section 1.6.2 for details). 


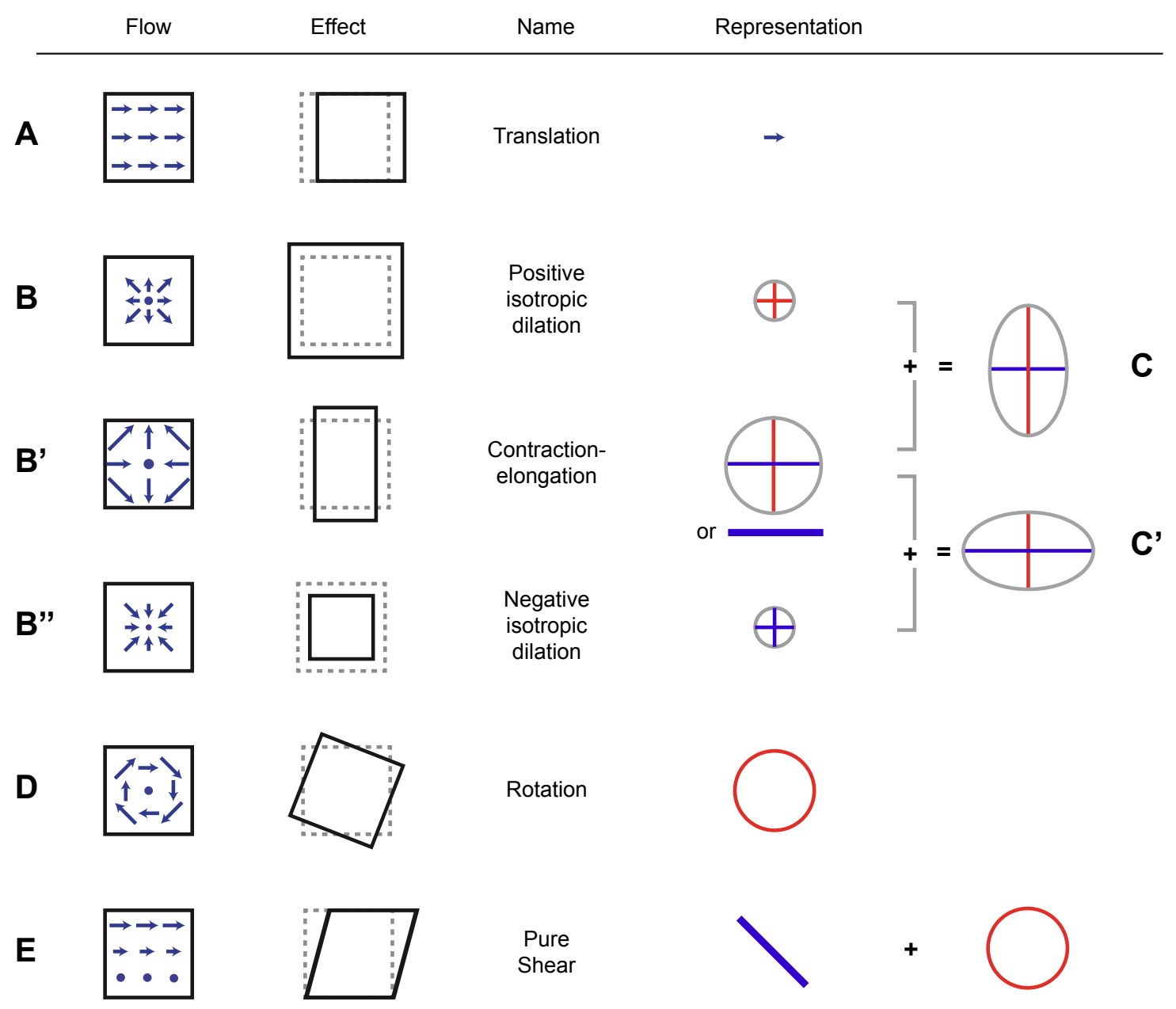

Figure S17. Local deformation rate, contraction-elongation rate, and rotation rate. The velocity gradient is decomposed into its symmetric and its anti-symmetric parts (31, 32). The deformation rate, related to the symmetric part of the velocity gradient, reflects changes in tissue size and shape. From it we separate the size changes to extract the contraction-elongation rate, which directly reflects effective changes in tissue shape. The local rotation rate or vorticity, related to the anti-symmetric part of the velocity gradient, does not by itself contribute to tissue shape change. Accordingly, contraction-elongation and rotation are two quantities that can vary independently. If the velocity gradient has no symmetric part, the tissue rotates as a whole, and there is no tissue shape change. If the rotation rate is spatially heterogeneous, the velocity gradient has non-zero symmetric and anti-symmetric parts: in that case there are simultaneously a shape change and a rotation, but they are not related, and again the rotation is not directly involved in tissue shape change. We thus purposedly exclude the rotation from our analysis of morphogenetic changes, and use it only to synchronise the movies (section 2.2.2 and fig. S4). To avoid ambiguities we do not use the words "shear rate" which in the literature indicate either a symmetrized (B') or a non-symmetrized (E) velocity gradient.

Schematic, not drawn to scale, of the notations and definitions used in the text. (A) A global translation of a tissue domain does not contribute to its shape or size change. (B-B") A positive isotropic dilation (B) has two equal positive eigenvalues (in red). A "contraction-elongation" (B') represents perpendicular contraction 
and elongation with the same amplitude: it has two opposite eigenvalues (red for positive, blue for negative), and can be represented indifferently by a red bar and a blue one within a circle or only by the blue bar for clarity. A negative isotropic dilation (B") has two equal negative eigenvalues (in blue). Dilations contribute to tissue size change, contraction-elongation to tissue shape change. (C-C') Combining a dilation and a contraction-elongation results in a deformation rate with a larger positive eigenvalue $\left(\mathrm{C}=\mathrm{B}+\mathrm{B}^{\prime}\right)$ or a larger negative eigenvalue (C'=B'+B'). Since B', C and C' correspond to the same contraction-elongation, they are represented by the same blue bar. (D) A rotation is represented by a circle, here red for clockwise (else, blue for counter-clockwise). It does not by itself contribute to tissue shape or size change. (E) "Pure shear" or "simple shear" can be decomposed in a contraction-elongation, which changes the shape, and rotation, which changes the orientation $\left(\mathrm{E}=\mathrm{B}^{\prime}+\mathrm{D}\right)$. The contraction-elongation is represented by a blue bar in the direction of contraction. For details see section 2.1.2.

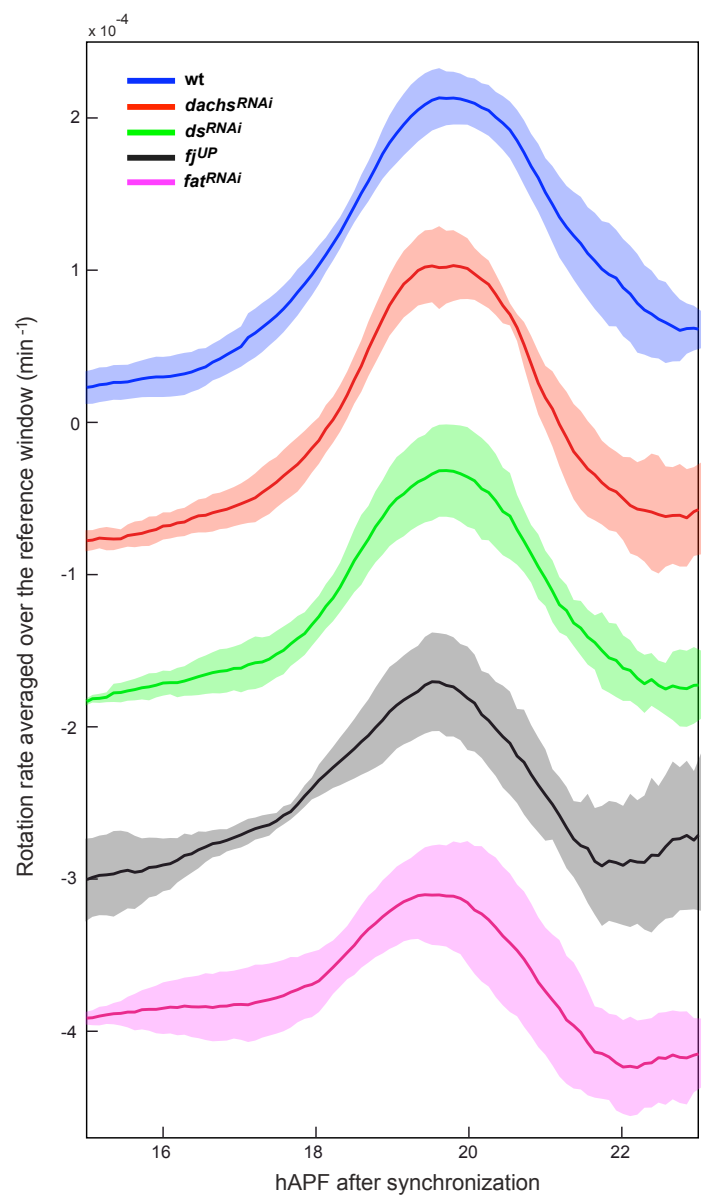

Figure S18. Rotation rate in wt and mutant scutella during morphogenesis. Qualitatively the peak of rotation rate in mutant scutella is the same as in wt, but quantitatively its amplitude is reduced.

Mean value (thick line) \pm ensemble standard deviation (shade) versus hAPF after synchronization. Tthe rotation rate was space averaged over the reference window (fig. S4) and ensemble averaged over $n$ movies of the same genotype. Blue, wt, $n=11$ (same data as fig. S4B"); red, dachs ${ }^{R N A i}, n=5$; green, $d s^{R N A i}$, $n=6$; black, $f j{ }^{U P}, n=4$; magenta, $f a t^{R N A i}, n=5$. Curves are vertically shifted by $10^{-4} \mathrm{~min}^{-1}$ for clarity. 


\subsection{Supporting movie captions}

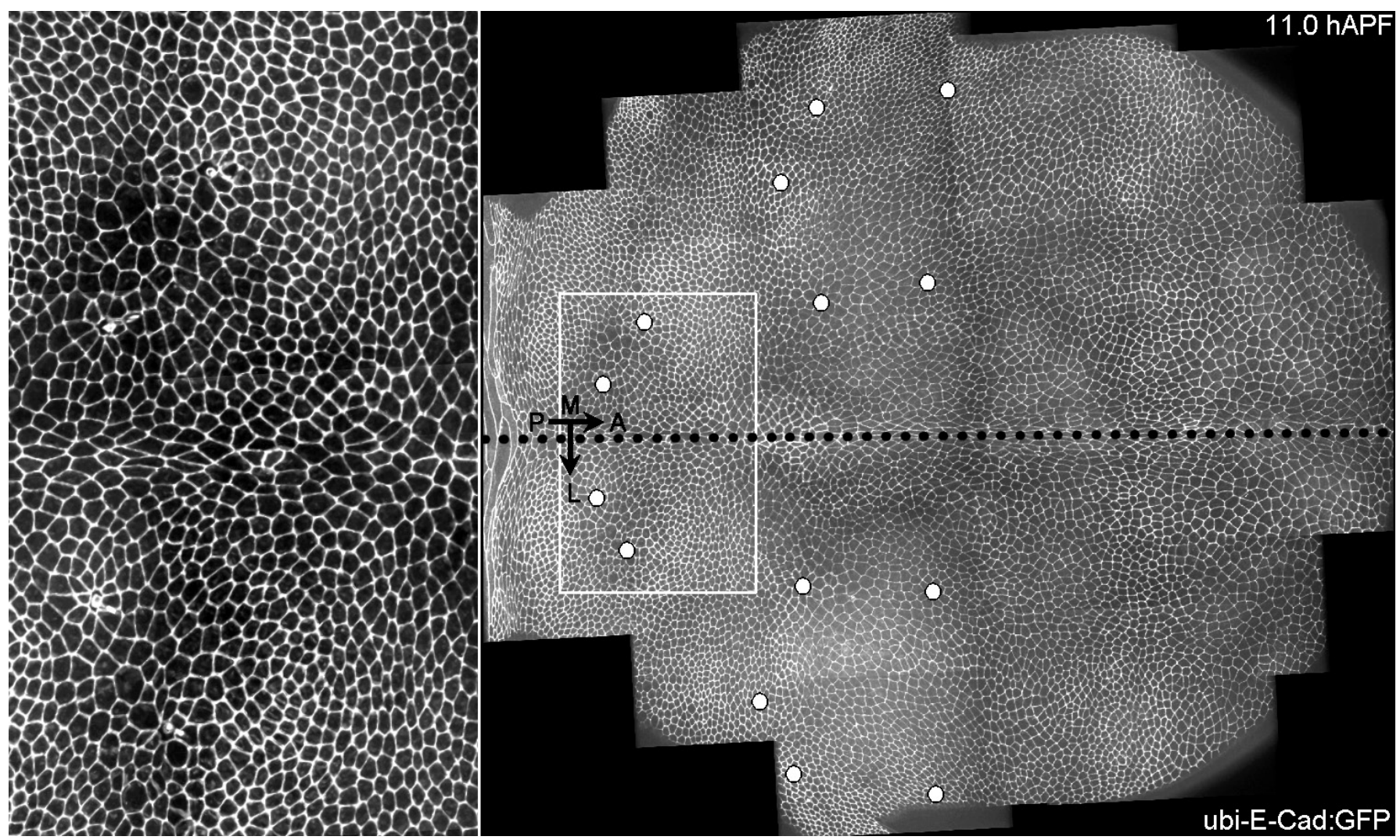

Movie S1. Drosophila pupa dorsal thorax tissue expressing E-Cad:GFP to label apical cell junctions, imaged by multi-position confocal microscopy (24 positions at a 5 min time resolution) between 11:0 and 36:2 hAPF. The boxed region, highlighting the scutellum, is magnified at the left. The positions of the macrochaetae and of the midline are indicated by white circles and by a black dotted line, respectively. Arrows indicate the $\mathrm{A} / \mathrm{P}$ and $\mathrm{M} / \mathrm{L}$ axes. For compression reasons, these images have half the resolution of original movie. 


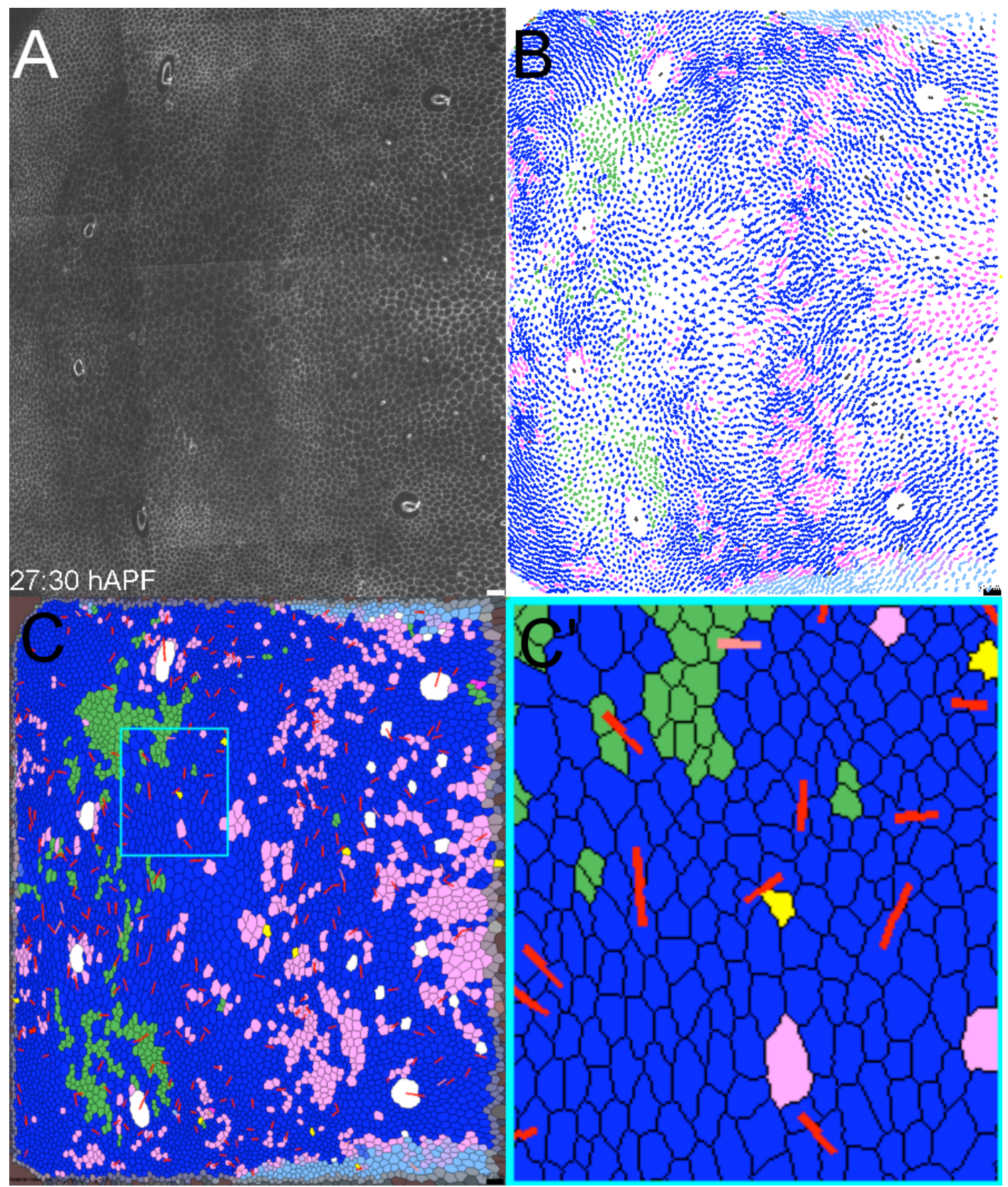

Movie S2. Tracking of cell dynamics in the scutellum and the posterior scutum. Note that, in order to show the full color code, the above still image is the last image of the movie. (A) Dorsal thorax tissue labeled with E-Cad:GFP and imaged by multi-position confocal microscopy at a 5 min time resolution between 11:30 and 27:30 hAPF. (B) Tracking of cell trajectories. (C) Tracking of cell divisions, apoptoses and rearrangements. Each cell is color coded according to the number of cell divisions that it undergoes during the movie: pink for cells having divided once; blue for cells having divided twice; green for the third wave of division. Links used to calculate cell rearrangement matrix (section 4.2) are shown as lines. Two cells which are in contact in an image, and not in the next one, correspond to a disappearing link, plotted as a yellow line. Two cells which get in contact in an image, and were not in contact in the previous one, correspond to an appearing link, plotted as a red line. Links involving a four-fold vertex are shown in lighter colours. Yellows zones indicate an apoptosis or delamination (which we do not distinguish). Light blue cells indicate new cells that entered the field of view. (C') Blow up of the region delimited in C by a cyan square. Scale bars: $10 \mu \mathrm{m}$. 


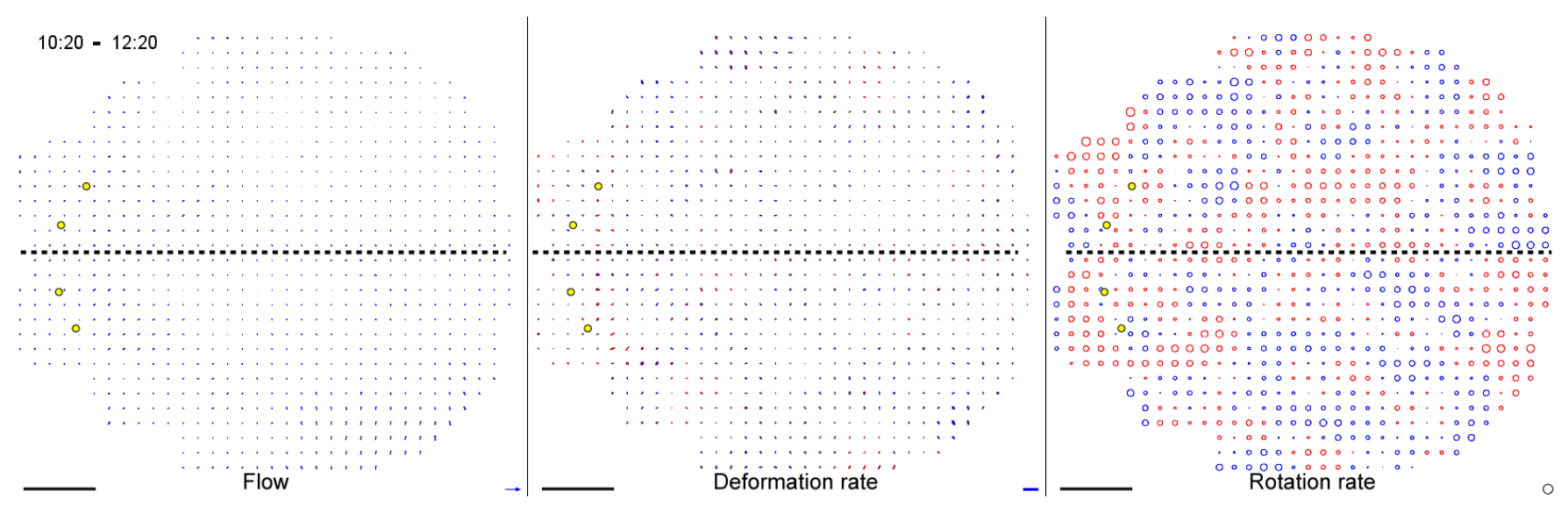

Movie S3. Local tissue flow, deformation rate and rotation rate measured on Movie S1. Left: flow velocity is represented as arrows. Middle: deformation rate is represented as ellipses; a direction of elongation is represented in red and contraction in blue. Right: rotation rate is represented by circle diameters; clockwise is represented in red and counter-clockwise in blue. For compression reasons, only one image every half hour is shown. Scale bars: $100 \mu \mathrm{m}$ (black bars), $910^{-2} \mu \mathrm{m} \mathrm{min} \mathrm{m}^{-1}$ (arrow, left), $2.410^{-3} \mathrm{~min}^{-1}$ (blue bar, middle), $810^{-5} \mathrm{~min}^{-1}$ (circle, right). 


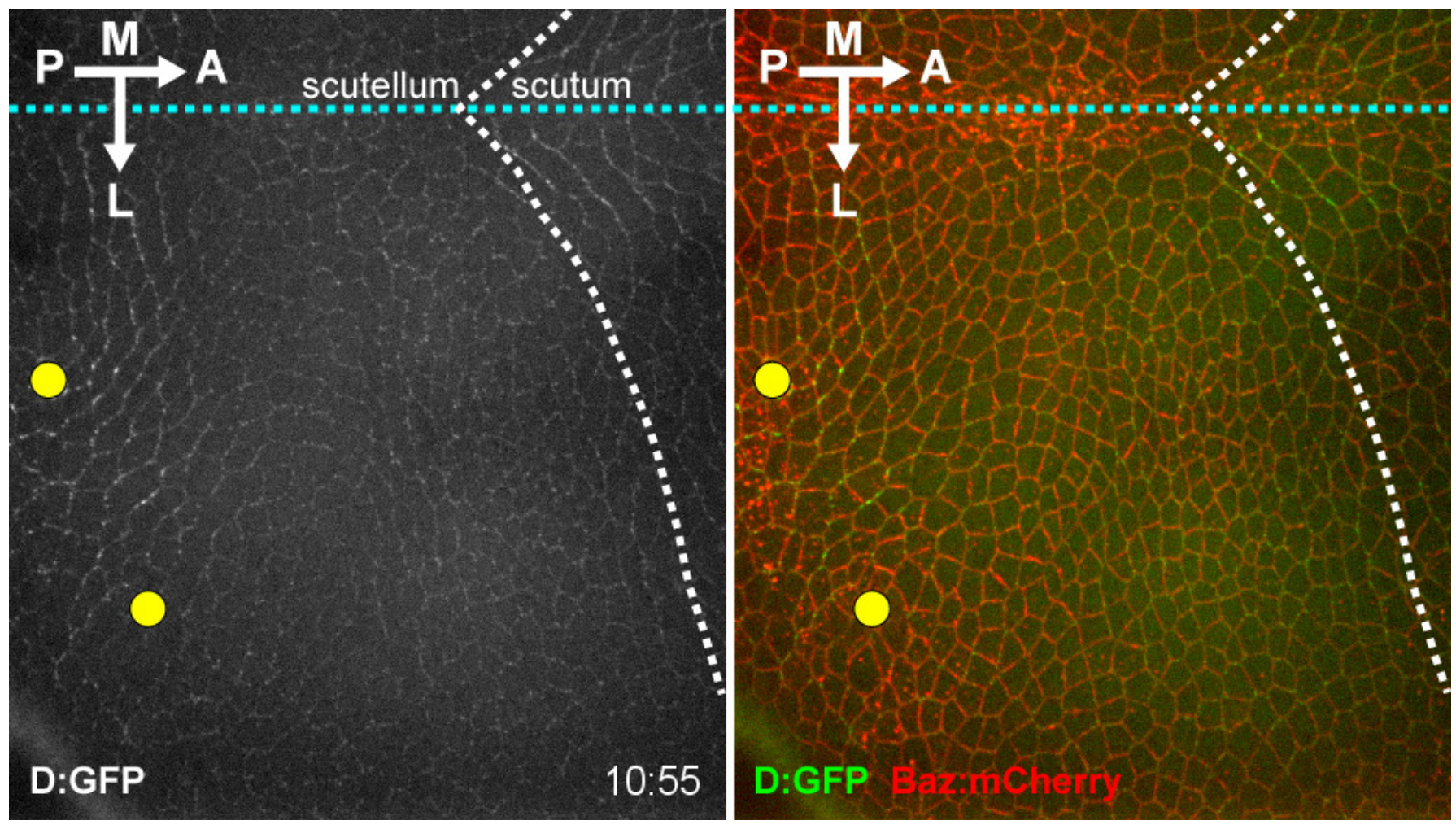

Movie S4. Scutellum labeled with D:GFP and Baz:mCherry during morphogenesis (11 to 26 hAPF). The positions of the macrochaetae and of the midline are indicated by yellow circles and by a cyan dashed line, respectively. Arrows indicate the $\mathrm{A} / \mathrm{P}$ and $\mathrm{M} / \mathrm{L}$ axes.

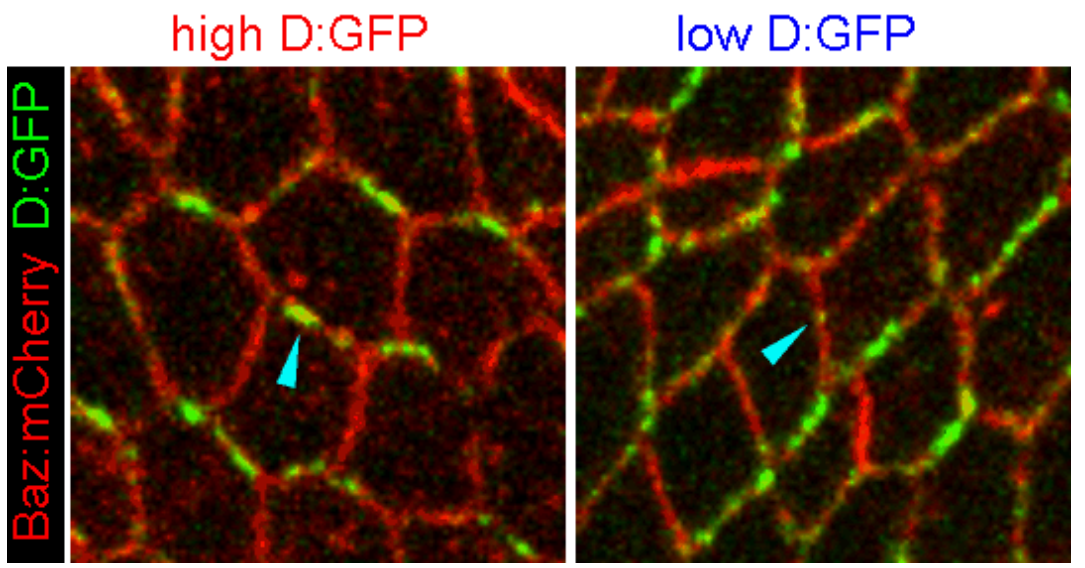

Movie S5. Representative movies of cell junction ablations with high (left) or low (right) D:GFP signal in the scutellum of pupae expressing D:GFP and Baz:mCherry. 


\section{References}

[1] H. Matakatsu, S. S. Blair, Interactions between Fat and Dachsous and the regulation of planar cell polarity in the Drosophila wing. Development 131, 3785-3794 (2004).

[2] M. P. Zeidler, N. Perrimon, D. I. Strutt, The four-jointed gene is required in the Drosophila eye for ommatidial polarity specification. Curr. Biol. 9, 1363-1372 (1999).

[3] H. Oda, S. Tsukita, Real-time imaging of cell-cell adherens junctions reveals that Drosophila mesoderm invagination begins with two phases of apical constriction of cells. J. Cell Sci. 114, 493-501 (2001).

[4] J. Huang, W. Zhou, W. Dong, A. M. Watson, Y. Hong, Directed, efficient, and versatile modifications of the Drosophila genome by genomic engineering. Proc. Natl. Acad. Sci. U. S. A 106, 8284-8289 (2009).

[5] M. H. Brodsky, H. Steller, Positional information along the dorsal-ventral axis of the Drosophila eye: graded expression of the four-jointed gene. Dev. Biol. 173, 428-446 (1996).

[6] Y. Mao et al., Dachs: an unconventional myosin that functions downstream of Fat to regulate growth, affinity and gene expression in Drosophila. Development 133, 2539-2551 (2006).

[7] E. Cho et al., Delineation of a Fat tumor suppressor pathway. Nat. Genet. 38, 1142-1150 (2006).

[8] A. Martin, M. Kaschube, E. Wieschaus, Pulsed contractions of an actin-myosin network drive apical constriction, Nature 457, 495-499 (2009).

[9] K. G. Golic, S. Lindquist, The FLP recombinase of yeast catalyzes site-specific recombination in the Drosophila genome. Cell 59, 499-509 (1989).

[10] T. Xu, G. M. Rubin, Analysis of genetic mosaics in developing and adult Drosophila tissues. Development 117, 1223-1237 (1993).

[11] K. Basler, G. Struhl, Compartment boundaries and the control of Drosophila limb pattern by hedgehog protein. Nature 368, 208-214 (1994).

[12] A. H. Brand, N. Perrimon, Targeted gene expression as a mean of altering cell fates and generating dominant phenotypes. Development 118, 401-415 (1993).

[13] S. E. McGuire, P. T. Le, A. J. Osborn, K. Matsumoto, R. L. Davis, Spatiotemporal rescue of memory dysfunction in Drosophila. Science 302, 1765-1768 (2003).

[14] K. J. Venken, Y. He, R. A. Hoskins, H. J. Bellen, P[acman]: a BAC transgenic platform for targeted insertion of large DNA fragments in Drosophila melanogaster. Science 314, 1747-1751 (2006).

[15] K. J. Venken et al., Versatile $\mathrm{P}$ [acman] BAC libraries for transgenesis studies in Drosophila melanogaster. Nat. Methods 6, 431-434 (2009).

[16] R. Benton, D. St Johnston, Drosophila PAR-1 and 14-3-3 inhibit Bazooka/PAR-3 to establish complementary cortical domains in polarized cells. Cell 115, 691-704 (2003). Erratum in Cell 116, 139 (2004). 
[17] J. Langevin et al., Lethal giant larvae controls the localisation of the Notch signalling regulators Numb, Neuralized and Sanpodo in Drosophila sensory organ precursor cells, Curr. Biol. 15, 955-962 (2005).

[18] M. Segalen et al., The Fz-Dsh planar cell polarity pathway induces oriented cell division via Mud/NuMA in Drosophila and Zebrafish. Dev. Cell 19, 740-752 (2010).

[19] H. Strutt, J. Mundy, K. Hofstra, D. Strutt, Cleavage and secretion are not required for Four-jointed function in Drosophila patterning. Development 131, 881-890 (2004).

[20] C. H. Yang, J. D. Axelrod, M. A. Simon, Regulation of Frizzled by fat-like cadherins during planar polarity signaling in the Drosophila compound eye. Cell 108, 675-688 (2002).

[21] D. Ma, C. H. Yang, H. McNeill, M. A. Simon, J. D. Axelrod, Fidelity in planar cell polarity signalling. Nature 421, 543-547 (2003).

[22] H. F. Clark et al., Dachsous encodes a member of the cadherin superfamily that controls imaginal disc morphogenesis in Drosophila. Genes Dev. 9, 1530-1542 (1995).

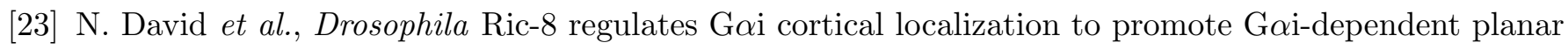
orientation of the mitotic spindle during asymmetric cell division. Nat. Cell Biol. 7, 1083-1090 (2005).

[24] M. S. Hutson et al., Plasma and cavitation dynamics during pulsed laser microsurgery in vivo. Phys. Rev. Lett. 99, 158104-158108 (2007).

[25] R. Farhadifar, J.-C. Röper, B. Aigouy, S. Eaton, F. Jülicher, The influence of cell mechanics, cell-cell interactions, and proliferation on epithelial packing. Curr. Biol. 17, 2095-2104 (2007).

[26] M. Rauzi, P. Verant, T. Lecuit, P.-F. Lenne, Nature and anisotropy of cortical forces orienting Drosophila tissue morphogenesis. Nat. Cell Biol. 10, 1401-1410 (2008).

[27] M. S. Hutson et al., Combining laser microsurgery and finite element modeling to assess cell-level epithelial mechanics. Biophys. J. 97, 3075-3085 (2009).

[28] M. Rauzi, P.-F. Lenne, Cortical forces in cell shape changes and tissue morphogenesis. Curr. Top. Dev. Biol. 95, 93-144 (2011).

[29] J. Boulanger et al., Patch-based nonlocal functional for denoising fluorescence microscopy image sequences. IEEE Trans. Med. Imaging 29, 442-454 (2010).

[30] G. Blanchard et al., Tissue tectonics: morphogenetic strain rates, cell shape change and intercalation. Nat. Methods 6, 458-464 (2009).

[31] Y.C. Fung, Biomechanics: mechanical properties of living tissues (Springer-Verlag, New York, 1993).

[32] G. K. Batchelor, An introduction to fluid dynamics (Cambridge Univ. Press, Cambridge, 2000).

[33] M. Raffel, C.E. Willert, S.T. Wereley, J. Kompenhans, Particle Image Velocimetry: A Practical Guide (Springer-Verlag, New York, 2007). 
[34] M. Gho, Y. Bellaïche, F. Schweisguth, Revisiting the Drosophila microchaete lineage: a novel intrinsically asymmetric cell division generates a glial cell. Development 126, 3573-3584 (1999).

[35] R. C. Gonzalez, R. E. Woods, S. L. Eddins, Digital Image processing using MATLAB (Gatesmark, ed. 2, 2009).

[36] T. Lecuit, P.-F. Lenne, Cell surface mechanics and the control of cell shape, tissue patterns and morphogenesis. Nature Rev. Mol. Cell Biol. 8, 633-644 (2007).

[37] P. Marmottant et al., The role of fluctuations and stress on the effective viscosity of cell aggregates Proc. Natl. Acad. Sci. USA, 106, 17271-17275 (2009).

[38] D. Ambrosi, L. Preziosi, Cell adhesion mechanisms and stress relaxation in the mechanics of tumours. Biomech. Model Mechanobiol. 8, 397-413 (2009).

[39] G. Forgacs, R. A. Foty, Y. Shafrir, M. S. Steinberg, Viscoelastic properties of living embryonic tissues: a quantitative study. Biophys. J. 74, 2227-2234 (1998).

[40] J. Ranft et al., Fluidization of tissues by cell division and apoptosis. Proc. Natl. Acad. Sci. USA. 107, 20863-20868 (2010).

[41] R. Foty, G. Forgacs, C. Pfleger, M. Steinberg, Liquid properties of embryonic tissues: measurement of interfacial tensions. Phys. Rev. Lett. 72 2298-2301 (1994).

[42] K. Guevorkian, D. Gonzalez-Rodriguez, C. Carlier, S. Dufour, F. Brochard-Wyart, Mechanosensitive shivering of model tissues under controlled aspiration. Proc. Natl. Acad. Sci. USA. 108, 13387-13392 (2011).

[43] L.D. Landau, E.M. Lifshitz, Fluid Mechanics (2nd ed.), Butterworth-Heinemann (Oxford, 1987).

[44] D. A. Reinelt, A. M. Kraynik, Simple shearing flow of dry soap foams with tetrahedrally close-packed structure, J. Rheol. 44, 453-471 (2000).

[45] F. Graner, B. Dollet, C. Raufaste, P. Marmottant, Discrete rearranging disordered patterns, part I: robust statistical tools in two or three dimensions. Eur. Phys. J. E 25, 349-369 (2008).

[46] B. Aigouy et al. Cell flow reorients the axis of planar polarity in the wing epithelium of Drosophila. Cell 142, 773-786 (2010).

[47] M. Aubouy, Y. Jiang, J. A. Glazier, F. Graner, A texture tensor to quantify deformations. Granular Matt. 5, 67-70 (2003).

[48] L. Butler et al., Cell shape changes indicate a role for extrinsic tensile forces in Drosophila germ-band extension. Nat. Cell Biol. 11, 859-864 (2009).

[49] D. Rogulja, C. Rauskolb, K. D. Irvine, Morphogen control of wing growth through the Fat signaling pathway. Dev. Cell 15, 309-321 (2008).

[50] H. Matakatsu, S. S. Blair, The DHHC palmitoyltransferase approximated regulates Fat signaling and Dachs localization and activity. Curr. Biol. 18, 1390-1395 (2008). 
[51] H. Strutt, D. Strutt, Nonautonomous planar polarity patterning in Drosophila: dishevelled-independent functions of frizzled. Dev. Cell 3, 851-863 (2002).

[52] R. Sopko et al., Phosphorylation of the tumor suppressor fat is regulated by its ligand Dachsous and the kinase discs overgrown. Curr. Biol. 19, 1112-1117 (2009).

[53] Y. Feng, K. D. Irvine, Processing and phosphorylation of the Fat receptor. Proc. Natl. Acad. Sci. USA. 106, 11989-11994 (2009).

[54] G. Tzolovsky, H. Millo, S. Pathirana, T. Wood, M. Bownes, Identification and phylogenetic analysis of Drosophila melanogaster myosins. Mol. Biol. Evol. 19, 1041-1052 (2002).

[55] A. Mehta et al., Myosin-V is a processive actin-based motor, Nature 400, 590-593 (1999).

[56] C. Veigel et al., The motor protein myosin-I produces its working stroke in two steps, Nature 398 530-532 (1999).

[57] P.-D. Coureux et al., A structural state of the myosin V motor without bound nucleotide, Nature 425, 419-423 (2003).

[58] E. De La Cruz, A. Wells, S. Rosenfeld, E. Ostap, L. Sweeney, The kinetic mechanism of myosin V, Proc. Natl. Acad. Sci. USA. 96, 13726-13731 (1999). 